\title{
Spectroscopic observations of PHz G237.01+42.50: A galaxy protocluster at $z=2.16$ in the Cosmos field ${ }^{\star}$
}

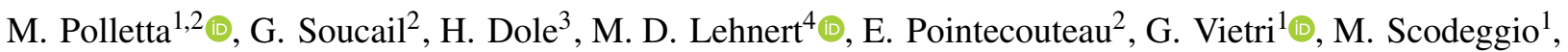

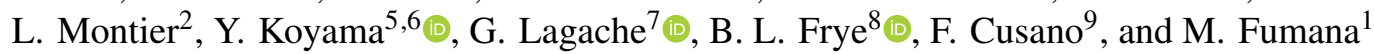 \\ (Affiliations can be found after the references)
}

Received 19 February 2021 / Accepted 2 August 2021

\begin{abstract}
The Planck satellite has identified more than 2000 protocluster candidates with extreme star formation rates (SFRs). Here, we present the spectroscopic identification of a Planck-selected protocluster located in the Cosmos field, PHz G237.01+42.50. PHz G237.01+42.50 contains a galaxy overdensity of 31 spectroscopically identified galaxies at $z \simeq 2.16$ (significant at $5.4 \sigma$ ) in a $10^{\prime} \times 11^{\prime}$ region. The overdensity contains two substructures or protoclusters at $\langle z\rangle \simeq 2.16$ and 2.195 with estimated halo masses at $z=0$ of $\sim 5-6 \times 10^{14} M_{\odot}$, roughly consistent with Virgo-type clusters. The overdensity total SFR, $4000 M_{\odot} \mathrm{yr}^{-1}$, is higher than predicted by simulations but much smaller than the SFR derived from the Planck data (i.e., $10173 \mathrm{M}_{\odot} \mathrm{yr}^{-1}$ ). The analysis of the Herschel data in the field, in combination with the available ancillary data, shows that such a difference is due to an effect of source alignment along the line of sight that produces a $5 \sigma$ overdensity of red Herschel sources in the field. We analyze the members' ultraviolet (UV) spectra and UV-far-infrared spectral energy distributions to derive their SFR, stellar mass, and metallicity. Galaxy members include blue star-forming galaxies and Active galactic nuclei (AGN) with SFRs and stellar masses consistent with the main sequence. Active galactic nuclei, identified through optical spectroscopy or X-ray data, represent a significant fraction $(20 \pm 10 \%)$ of all members of the protocluster at $z=2.16$, and they are powerful enough to produce radiative feedback. The core of this protocluster, besides being denser, includes members that are, on average, more massive and star-forming and contains a larger fraction of AGN and Herschel-detected galaxies than the full sample, suggesting an environmental effect on galaxy growth. A comparison between $\mathrm{PHz}$ G237.01+42.50 and other protoclusters in the literature at similar redshifts reveals some common traits and differences that reflect both observational biases and a diversity in intrinsic properties that is not yet fully understood.
\end{abstract}

Key words. large-scale structure of Universe - galaxies: star formation - galaxies: clusters: general - galaxies: high-redshift submillimeter: galaxies

\section{Introduction}

The study of galaxy cluster progenitors, so-called protoclusters, allows us to investigate the growth of the most massive halos, the evolution of the large-scale structure at high redshift, the history of star formation in dense environments, and the link between galaxy evolution and environment (Kravtsov \& Borgani 2012; Overzier 2016). Finding and studying high- $z$ growing structures is challenging as protoclusters are rare (Boylan-Kolchin et al. 2009; Overzier et al. 2009), necessitating either wide area surveys or surveys that increase the volume probed by pushing to the highest redshifts.

In the past few years, the search and study of the earliest structure has yielded hundreds of candidates and a dozen protoclusters studied in detail (e.g., Pentericci et al. 2000; Matsuda et al. 2005; Chapman et al. 2009; Galametz et al. 2010; Capak et al. 2011; Hatch et al. 2011; Tanaka et al. 2011; Koyama et al. 2013; Altieri \& Dannerbauer 2014; Cooke et al. 2014; Cucciati et al. 2014; Lemaux et al. 2014; Diener et al. 2015; Chiang et al. 2015; Casey et al. 2015; Wang et al. 2016; Noirot et al. 2016, 2018). These protoclusters have been discovered as overdensities of star-forming galaxies (SFGs) identified through photometric (e.g., Wang et al. 2016) or spectroscopic surveys (e.g., Lemaux et al. 2014; Steidel et al. 2005; Chiang et al. 2014), that often target sources known to be tracers of dense environments, such as high- $z$ radio galaxies (HzRGs), quasi stel-

^ Based on LBT/LUCI spectroscopic observations. lar objects (QSOs), and sub-millimeter (sub-mm) galaxies (e.g., Ivison et al. 2013; Dannerbauer et al. 2014; Cooke et al. 2015; Casey et al. 2015; Casey 2016; Venemans et al. 2007; Kuiper et al. 2012; Hayashi et al. 2016; Noirot et al. 2018).

Different selection techniques have yielded a variety of protocluster candidates whose members are often biased by the identification method. These biases hinder a proper comparison and obtaining a global picture of their properties and of their galaxy members.

Ideally, we wish to have a homogeneous sample of protoclusters covering a wide range of properties, such as redshift, predicted final cluster mass, total star formation rate (SFR), and size. For each protocluster it would be desirable to have an unbiased census of their members by identifying them in an unbiased way as well as to know their physical properties (stellar mass, SFR, cold gas mass, morphology). With such a sample it would then be possible to investigate which processes play a dominant role in galaxy growth and evolution in forming structures.

Many searches at $z>1.5$ have been carried out at sub$\mathrm{mm}$ wavelengths as a large fraction of member galaxies are expected to undergo a dust-obscured star-formation phase (e.g., Dannerbauer et al. 2014; Clements et al. 2016; Kato et al. 2016; Wagner et al. 2017; Nantais et al. 2017). Indeed several sub-mm bright protoclusters have been discovered, at small $(\sim 100 \mathrm{kpc}$; Ivison et al. 2013; Gómez-Guijarro et al. 2019; Wang et al. 2016; Miller et al. 2018), medium ( $500 \mathrm{kpc}$; Oteo et al. 2018; Lacaille et al. 2019), and large (several megaparsecs; 
Dannerbauer et al. 2014; Casey et al. 2015; Kato et al. 2016; Hung et al. 2016; Umehata et al. 2019) scales.

Negrello et al. (2005) predict the detection by Planck ${ }^{1}$ (Planck Collaboration I 2011) and the Herschel Space Observatory $^{2}$ (Pilbratt et al. 2010) sub-mm surveys of unresolved intensity peaks made by the summed emission of dusty star-forming high- $z$ protocluster members within the arcminute-sized beam (arcminute scale). For these reasons, we embarked on an ambitious program to identify protoclusters based on their overall overdensity of ongoing star formation. We used the Planck satellite to search for high- $z$ structures in a systematic way over the cleanest $26 \%$ of the extragalactic sky, at an angular resolution well matched to the expected size of these structures $(5 \mathrm{arcmin}$, or $\sim 6 \mathrm{Mpc}$ in comoving scales at $z \simeq 2-3$; Muldrew et al. 2015). The Planck telescope, due to its sensitivity at sub-mm wavelengths where SFGs at $z \gtrsim 2$ are bright, has provided a homogeneously selected sample of 2151 protocluster candidates at redshifts $\simeq 2-4$ - the Planck high- $z$ (PHz) sample (Planck Collaboration Int. XXXIX 2016). The sample selection and main properties are summarized in Sect. 2. The large sub-mm fluxes we see in the $\mathrm{PHz}$ fields can be due to statistical fluctuations of the cosmic IR background, single strongly lensed galaxies (Cañameras et al. 2015), overdensities of bright SFGs in the early Universe (Planck Collaboration Int. XXVII 2015), or projections along the line of sight of bright galaxies or of structures at different redshifts (Negrello et al. 2017). The most active star-forming protoclusters must be among the brightest sub-mm sources of the sky and are thus expected to be part of this sample. Follow-up studies of $\mathrm{PHz}$ protocluster candidates are relevant for a statistical characterization of a large and homogeneous protocluster sample, as well as for the discovery of extreme objects.

High- $z$ protoclusters offer also the possibility of investigating several questions that are relevant in cosmology and galaxy evolution, for example the processes that regulate the intense star formation observed in these structures, the mechanisms responsible for their subsequent quenching, and the role of the activity associated with active galactic nuclei (AGN).

In this work, we present the spectroscopic confirmation of the only $\mathrm{PHz}$ source located in the Cosmos field (Scoville et al. 2007) for which spectroscopic observations with the Large Binocular Telescope Near-infrared Spectroscopic Utility with Camera and Integral Field Unit for Extragalactic Research (LUCI) spectrograph (Seifert et al. 2003; Buschkamp et al. 2012) on the Large Binocular Telescope (LBT; Hill et al. 2012) have been obtained, $\mathrm{PHz}$ G237.01+42.50 (G237). The advantage of using this field is the availability of a wealth of multiwavelength data, in particular of spectroscopic redshifts, that allow us to identify and analyze the protocluster member galaxies.

Throughout this work we use magnitudes in the AB system, and adopt a Chabrier (2003) initial mass function (IMF). We denote the stellar mass with $\mathcal{M}$ and the characteristic stellar mass with $\mathcal{M}^{*}$. We assume a flat $\Lambda$ cold dark matter (CDM) model, with cosmological parameters from the Planck 2018 release, $\Omega_{\Lambda}=0.685, \Omega_{\mathrm{m}}=0.315, \mathrm{H}_{0}=67.4 \mathrm{~km} \mathrm{~s}^{-1} \mathrm{Mpc}^{-1}$ (Planck

\footnotetext{
1 Planck (http://WwW.esa.int/Planck) is a project of the European Space Agency (ESA) with instruments provided by two scientific consortia funded by ESA member states (in particular the lead countries France and Italy), with contributions from NASA (USA), and telescope reflectors provided by a collaboration between ESA and a scientific consortium led and funded by Denmark.

2 Herschel is an ESA space observatory with science instruments provided by the European-led Principal Investigator consortia and with important participation from NASA.
}

Collaboration VI 2020). We express physical and comoving distances in units of pkpc and ckpc, respectively. With the adopted cosmology, $1^{\prime \prime}$ corresponds to a scale of $\sim 8.5 \mathrm{pkpc}$, and $18.1 \mathrm{ckpc}$ at $z=2.16$.

\section{The Planck high-z source sample}

The PHz catalog (Planck Collaboration Int. XXXIX 2016) was extracted from $26 \%$ of the Planck all sky maps. The cata$\log$ includes all sources that were detected at more than $5 \sigma$ in the red-excess (RX) map. The RX map is obtained after subtracting from the map at $550 \mu \mathrm{m}$ the image obtained by linearly interpolating the signal in the $350 \mu \mathrm{m}$, and $850 \mu \mathrm{m}$ maps. The selection requires a signal at more than $3 \sigma$ in the cleaned $^{3}$ maps at 350,550 , and $850 \mu \mathrm{m}$, and a non-detection $(S / N<3)$ in the $100 \mathrm{GHz}$ map. In order to favor only reliable high- $z$ candidates, all selected sources must also satisfy a color selection criterion. More specifically, a flux ratio $S_{550} / S_{350}>$ 0.5 is required to reduce contamination from Galactic cold sources, and $S_{850} / S_{550} \lesssim 0.9$ to avoid contamination from radio and Sunyaev-Zel'dovich sources (see Fig. 15 in Planck Collaboration Int. XXXIX 2016). The PHz catalog contains 2151 sources. The selection procedure and the catalog are presented in Planck Collaboration Int. XXXIX (2016). The color selection criteria favor sources at $1.5<z<4$ assuming realistic values of dust temperature and emissivity index. The low angular resolution of the Planck beam $(\mathrm{FWHM}=4.3 \mathrm{arcmin}$ at $550 \mu \mathrm{m}$ ) and the $90 \%$ completeness limit of $\sim 500 \mathrm{mJy}$ in the submm imply that the $\mathrm{PHz}$ sources correspond to environments of typically $2 \mathrm{Mpc}$ in size with $L_{I R} \sim 3 \times 10^{13} L_{\odot}$, for $z \sim 2-3$.

Identifying and characterizing these sources require multiwavelength deep photometric data over wide regions. Several follow-up observations have been carried out to obtain multiwavelength data for a representative subsample. First, sub-mm observations with the Spectral and Photometric Imaging REceiver (SPIRE; Griffin et al. 2010) instrument on the Herschel Space Observatory have been obtained for $228 \mathrm{PHz}$. Of these, 217 targets (95\%) were found to correspond to overdensities of bright $\left(S_{350 \mu \mathrm{m}}>37 \mathrm{mJy}\right)$ and $\mathrm{red}^{4}$ SPIRE sources (Planck Collaboration Int. XXVII 2015). Spitzer/IRAC imaging at 3.6 and $4.5 \mu \mathrm{m}$ have been obtained for 92 sources out of the Herschel subsample (Martinache et al. 2018). Optical and nearinfrared (NIR) imaging data have been obtained for $42 \mathrm{PHz}$. Spectroscopic observations, necessary to confirm the protocluster nature of these sources, have been obtained only for three $\mathrm{PHz}, \mathrm{PHz}$ G95.5-61.6, resulting in the identification of two potential structures, one at $z \simeq 2$ with six members, and a second one at $z \simeq 1.7$ with three members (Flores-Cacho et al. 2016), and $\mathrm{PHz}$ G237.01+42.50, whose observations are presented here. A third PHz, PLCK G073.4-57.5, has been observed with ALMA, yielding two CO line detections at the same redshift, $z=1.54$ (Kneissl et al. 2019). The paucity of spectroscopic observations is due to the complication of identifying the counterpart to the sub-mm sources, the need of data over wide enough areas to cover the extension of the $\mathrm{PHz}$, and the difficulty of obtaining redshifts for galaxies at $z \sim 2$, in particular, for dusty star-forming galaxies (DSFGs; Casey et al. 2017).

\footnotetext{
3 The cleaned maps are obtained after removing the signal from the cosmic microwave background (CMB), and Galactic cirrus emission.

4 A SPIRE source is defined red if detected in all three SPIRE bands and with $S_{500} / S_{350}>0.6$ and $S_{350} / S_{250}>0.7$ (see Planck Collaboration Int. XXVII 2015).
} 
In addition to the data obtained through our follow-up campaign, we looked for $\mathrm{PHz}$ sources located in public legacy fields, in particular in the $>50 \mathrm{deg}^{2}$ HerMES fields (Oliver et al. 2012), where a large set of multiwavelength data, including Herschel SPIRE data, are available. Based on the density of the $\mathrm{PHz}$ sources (i.e., $0.2 \pm 0.004$ sources $\mathrm{deg}^{-2}$ ), we expect $20 \pm 4 \mathrm{PHz}$ in the HerMES fields $\left(99.5 \mathrm{deg}^{2}\right)$. There are 21 (1\% of the full sample) $\mathrm{PHz}$ in HerMES, and one, $\mathrm{PHz}$ G237.01+42.50, in the $1.6 \mathrm{deg}^{2}$ Cosmos field, where, in addition to multiwavelength photometric data, high-resolution imaging and spectroscopic data are available.

\section{PHz G237.01+42.50: A Planck structure in the Cosmos field}

$\mathrm{PHz} 237.01+42.50$ (G237 hereinafter) is the only $\mathrm{PHz}$ source located in the Cosmos field. Its Planck position is $\alpha=150.507 \mathrm{deg}$, and $\delta=2.31204 \mathrm{deg}$, and size is $\sim 12^{\prime} \times 5.5^{\prime}$. Its total Planck fluxes are $S_{550 \mu \mathrm{m}}=0.61 \pm 0.04 \mathrm{Jy}$, and $S_{350 \mu \mathrm{m}}=0.69 \pm 0.03 \mathrm{Jy}$, and the signal-to-noise ratios $(\mathrm{S} / \mathrm{Ns})$ at 850,550 and $350 \mu \mathrm{m}$, and in the RX $550 \mu \mathrm{m}$ map, are 5.2, 5.8, 3.5, and 6.0, respectively, making G237 among the $30 \%$ most significant detections in the $\mathrm{PHz}$ sample. Based on these flux densities, and assuming a modified black body model with temperature of $30 \mathrm{~K}$ at $z=2.2$, and the Kennicutt (1998) relation between the IR luminosity and the SFR, the estimated SFR of G237 is $18210 M_{\odot} \mathrm{yr}^{-1}$ (assuming a Salpeter $1955 \mathrm{IMF}$ and $10173 M_{\odot} \mathrm{yr}^{-1}$ assuming a Chabrier 2003 IMF; Planck Collaboration Int. XXXIX 2016).

\subsection{LBT spectroscopic observations}

As part of our follow up program, we obtained spectroscopic observations of G237 (Italian Program 34; PI: Polletta) with LUCI (Seifert et al. 2003, 2010; Ageorges et al. 2010; Buschkamp et al. 2012) on the $8.4 \mathrm{~m}$ LBT (Hill et al. 2012) in monocular mode. LUCI provides NIR multiobject spectroscopy over a field of view of $4^{\prime} \times 4^{\prime}$. We used the N1.8 camera $\left(0.249^{\prime \prime}\right.$ pixel $\left.^{-1}\right)$ and the second order of the $200 \mathrm{H}+\mathrm{K}$ grating with a central wavelength of $1.93 \mu \mathrm{m}$, providing a resolving power of $R \sim 1100$. The wavelength coverage is slightly different from object to object (depending on the position of the slit in the mask) but typically spans $1.50-2.30 \mu \mathrm{m}$. We observed 15 targets with $\mathrm{AB} K$-band mag $\leq 22$ selected from the ultraVISTA 3rd data release catalog (McCracken et al. 2012). Five of them are within $11^{\prime \prime}$ of a SPIRE source, our highest priority sources, the remaining ten are filler sources selected because of their red optical-NIR colors. The layout of the LUCI mask overlaid on the UltraVISTA $K_{s}$ image of G237 is shown in Fig. 1. The observations were conducted on 2016 April 21-22. Flats and arcs were taken on the same day of the observations. The observing conditions were good with mostly clear sky, an airmass of 1.28 , on average, and a seeing between 0.5 and $1.25^{\prime \prime}\left(0.9^{\prime \prime}\right.$, on average). The observations were divided into five observing blocks, with twenty 120 s exposures each, for a total exposure time of $3 \mathrm{~h} 20 \mathrm{~min}$. We used slit widths of $1^{\prime \prime}$, and slit length of either 8 or $10^{\prime \prime}$ to sample enough sky background for sky subtraction. The nodding technique was applied to obtain a good quality sky subtraction during the reduction phase. A telluric star was observed at the end of each night for the flux calibration and for correction of atmospheric absorption.

The data reduction procedure was carried out by the Italian LBT support team using a dedicated pipeline developed at INAF

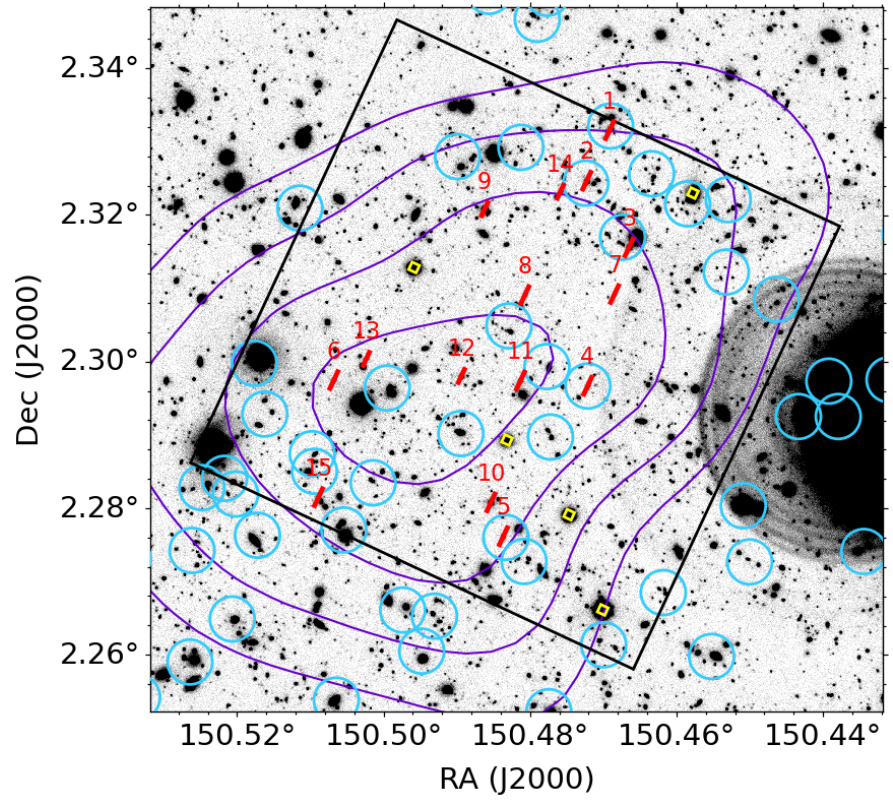

Fig. 1. Layout of the LUCI mask (black square: $4^{\prime} \times 4^{\prime}$ ) overlaid on the UltraVISTA $K_{s}$-band $6^{\prime} \times 6^{\prime}$ image. The slits assigned to the spectroscopic targets are shown as red rectangles $\left(1^{\prime \prime} \times 8-10^{\prime \prime}\right)$ and identified by the slit number, alignment stars are shown by yellow square. The SPIRE sources positions are indicated by blue circles (11" in radius). Planck red-excess contours (at 50, 62.5, 75 and $87.5 \%$ of the highest value) are shown as purple lines. North is up and east to the left.

IASF-Milan (Scodeggio et al. 2005; Garilli et al. 2012). This includes flat-fielding, sky subtraction, correction for distortions, and wavelength and flux calibration. The background was subtracted from frames in pairs obtained from nodding along the slit. Wavelength calibration was carried out using $\mathrm{OH}$ sky lines, and flux calibration using a telluric standard star. Monodimensional spectra extraction was performed using the Horne extraction algorithm (Horne 1986). The final products include sky and noise spectra, and flux calibrated bi- and mono-dimensional spectra. The spectra of the 15 observed objects are shown in Fig. A.1. Redshifts were determined using the EZ code (Garilli et al. 2010).

The complete list of spectroscopic targets, with coordinates, estimated redshifts and associated quality flags, $K$-band magnitudes, and IRAC colors from the Cosmos multiwavelength cata$\log$ (Laigle et al. 2016; L16 hereinafter) are given in Table A.1. We were able to obtain a reliable redshift $(z f l g>1)$ estimate for five sources, of which two are at the same redshift, $z \simeq 2.16$. One of these two objects (ID SL01) exhibits a broad $\mathrm{H} \alpha$ line $\left(F W H M=4004 \pm 327 \mathrm{~km} \mathrm{~s}^{-1}\right)^{5}$ and it is thus classified as broad line AGN (Khachikian \& Weedman 1974).

After analyzing these data, we discovered that six of our selected LUCI targets had been previously observed by the Cosmos team and, for five of them, a spectroscopic redshift was available from the zCosmos-Deep survey (Lilly et al. 2009). These redshift are listed in Table A.1, and relative spectra are shown in Fig. A.2. In Fig. A.1, we show the expected spectral features (see red vertical dashed line) in the LUCI NIR spectra assuming the zCosmos-Deep redshift. In only one case we have a

\footnotetext{
5 The width of the broad $\mathrm{H} \alpha$ line has been derived after fitting the restframe spectrum in the $6400-6750 \AA$ wavelength range with a power-law to model the continuum, and four Gaussian components to model the narrow, and broad $\mathrm{H} \alpha$ lines, and the N II $\lambda 6584$ doublet.
} 
redshift estimate from both LUCI and zCosmos, ID slit08. They differ significantly, the LUCI estimate, $z=1.52$, is highly reliable $(z$ flag $=4)$, while the zCosmos one, $z=0.86$, is uncertain $(z$ flag $=1)$. In the other four cases, there are no strong features observable in the NIR, thus the spectra are consistent with the zCosmos redshift estimates.

Since the chances of finding two sources at the same redshift out of such a small sample are small, we investigate the hypothesis that G237 might contain a structure at such a redshift. To this end we searched for all the available spectroscopic redshifts in a wide region centered on G237, and analyzed its distribution, as described in the next section.

\subsection{Cosmos spectroscopic redshifts}

We collected a large number of spectroscopic redshifts in a region $\sim 10^{\prime}$ wide centered on G237 from various public surveys (zCosmos Bright; Lilly et al. 2007; FMOS; Silverman et al. 2015; Magellan; Trump et al. 2007; Spitzer/IRS; Fu et al. 2010; DEIMOS; Hasinger et al. 2019), and from the zCosmos-Deep survey (Lilly et al. 2009). From this compilation, we obtained the redshift distribution in the $10^{\prime} \times 12^{\prime}$ region centered on G237 (see Fig. 2). The distribution shows two narrow peaks, one at $z_{\text {peak }} \sim 2.155$ with 15 members, and another at $z_{\text {peak }} \sim 2.195$ with eight members where $\left|z-z_{\text {peak }}\right| /\left(1+z_{\text {peak }}\right)<0.0016$ (or $\left.|\Delta v| \leq 480 \mathrm{~km} \mathrm{~s}^{-1}\right)$. Six sources have intermediate redshifts, $2.16<z<2.19$. To quantify the significance of the two redshift peaks, we computed the median spectroscopic redshift distribution in a large region of the Cosmos field where the density of sources with a spectroscopic redshift is similar to the G237 field (i.e., $149.74^{\circ}<\alpha<150.65^{\circ}$, and $1.77^{\circ}<\delta<2.65^{\circ}$ ). Within such a region, we defined $2010^{\prime} \times 12^{\prime}$ boxes, and derived, in each box, the redshift distribution from $z=1.5$ to 2.8 with redshift bins of 0.01 . For each redshift bin, we computed the median number of sources, the mean absolute deviation from the median, and the 16th and 84th percentile values, after normalizing the number of sources with spectroscopic redshift per box to the number of sources found in the G237 region. The derived median distribution is shown in Fig. 2. Based on this distribution, we would expect $12.8 \pm 3.7,3.6 \pm 1.7$, and $2.1 \pm 1.5$ sources at $2.15 \leq z \leq 2.20,2.15 \leq z \leq 2.16$, and $2.19 \leq z \leq 2.20$, respectively. Compared to the number of sources in these redshift bins in G237, 29, 15, and 8, respectively, the significances of the redshift peaks in the three bins are 4.4, 6.8, and 4.0 $\sigma$, respectively. We can thus conclude that there is a significant overdensity of sources in all three redshift bins. Including the two sources from our LUCI sample, there are in total 31 sources with $2.15 \leq z_{\text {spec }}<2.20$ or $|\Delta v| \leq 2360 \mathrm{~km} \mathrm{~s}^{-1}$, yielding an overdensity contrast $\delta_{\text {gal }}{ }^{6}=1.3$, and 3.15, with a significance of $4.9 \sigma$ at $2.15 \leq z \leq 2.20$, and of $8 \sigma$ at $2.15 \leq z \leq 2.16$, respectively.

The list of 31 sources is reported in Table 6, and their spectra are shown in Figs. B.1, and B.2. We note that in 11 out of 29 objects the redshift estimate is highly uncertain (quality flag equal to 1), but after a careful check where we investigated alternative redshift solutions using the EZ code (Garilli et al. 2010), we concluded that no revision was better than the available estimate. The detection of two sources at $z \simeq 2.16$ in our LBT observations and the presence of a peak at the same redshift in the G237 field suggest that G237 contains a structure at such a

\footnotetext{
6 The overdensity contrast is defined as $\delta_{\text {gal }}=\left(\rho_{\text {source }}-\rho_{\text {field }}\right) / \rho_{\text {field }}$, where $\rho_{\text {source }}$ is the galaxy density in the source, and $\rho_{\text {field }}$ that in the field. The overdensity significance is defined as $\left(\rho_{\text {source }}-\rho_{\text {field }}\right) / \sigma_{\text {field }}$, where $\sigma_{\text {field }}$ is the galaxy density standard deviation in the field.
}

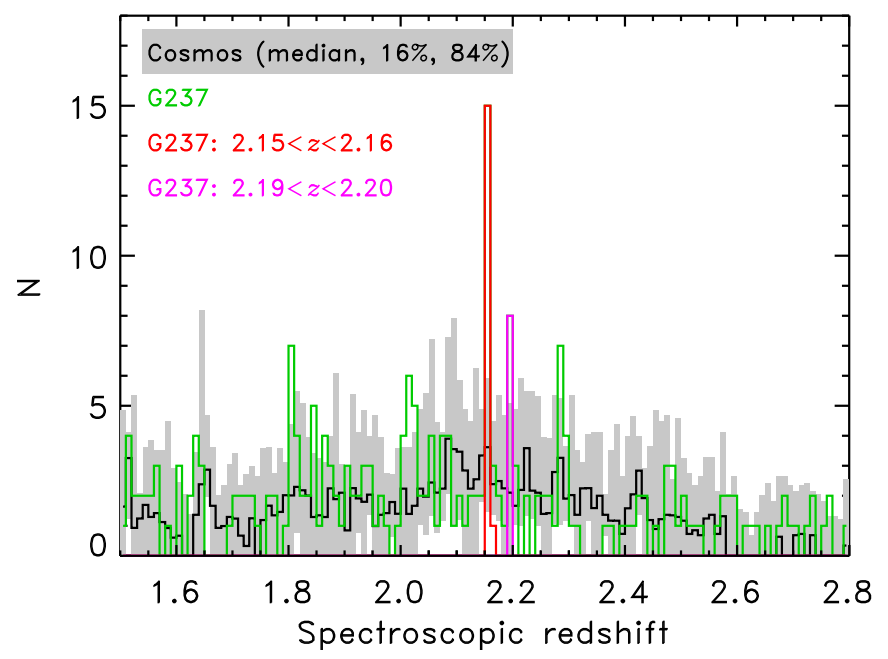

Fig. 2. Spectroscopic redshift distribution of all sources with known spectroscopic redshift from the Cosmos public redshift release and the zCosmos-Deep catalog within a $10^{\prime} \times 12^{\prime}$ region centered at $\alpha=150.465^{\circ}$, and $\delta=2.31^{\circ}$ where G237 is located (green line). Sources with $z=2.15-2.16$ are shown with a red line, and those with $z=2.19$ 2.20 with a magenta line. The redshift distribution from the same spectroscopic redshift list obtained by taking the median values from 20 non overlapping $10^{\prime} \times 12^{\prime}$ regions within the Cosmos field is shown as black solid line, with associated 16th and 84th percentile values (gray region).

redshift. To investigate this hypothesis further we examine the spatial distribution, both projected on the sky and along the line of sight of all the spectroscopic sources at $z \simeq 2.15-2.20$ (corresponding to $\Delta v=4646 \mathrm{~km} \mathrm{~s}^{-1}$ ).

\section{3. $\mathrm{H} \alpha$ emitters in $\mathrm{G} 237$}

The spectroscopic redshift peak found in G237 prompted us to carry out narrowband imaging observations to identify the $\mathrm{H} \alpha$ emitters (HAEs) in the field at $z \simeq 2.16$. MOIRCS/Subaru (Ichikawa et al. 2006) observations of G237 using the NB2071 filter $\left(\lambda_{c}=2.068 \mu \mathrm{m}, \Delta \lambda=0.027 \mu \mathrm{m}\right.$; corresponding to the $\mathrm{H} \alpha$ line at $z=2.13-2.17$ ) yielded the discovery of 38 HAEs (Koyama et al. 2021). The spatial distribution of all spectroscopic members, and the HAEs is shown in Fig. 3. Six out of eight spectroscopically confirmed galaxies with $z<2.17$ in the observed MOIRCS $4^{\prime} \times 7^{\prime}$ field are also selected as HAEs (see list in Table 6). The two spectroscopic members that are not detected as HAE (IDs 58173, and 57730) might have an $\mathrm{H} \alpha$ line with a small equivalent width (EW) (i.e., <30 $)$ ), or a wrong redshift.

The MOIRCS observations reveal a strong overdensity of HAEs, with a clear enhancement of massive HAEs at its peak. The density peaks of both, the spectroscopic and the HAE samples, are only $38^{\prime \prime}$ apart. This region will be identified as the protocluster core in the next section.

\subsection{Structure components}

The spatial distribution of all 31 sources at $2.15 \leq z<2.20$ in the PHz G237.01+42.50 field is shown in Fig. 4. We also show the projected distance along the line of sight in terms of velocity, where $100 \mathrm{~km} \mathrm{~s}^{-1}$ at these redshifts correspond to $\sim 1.24 \mathrm{cMpc}$. In Fig. 5, their distribution in transverse and radial distance is shown. The distribution of velocity offsets of all 31 sources is not Gaussian, indicating velocity substructures (see 


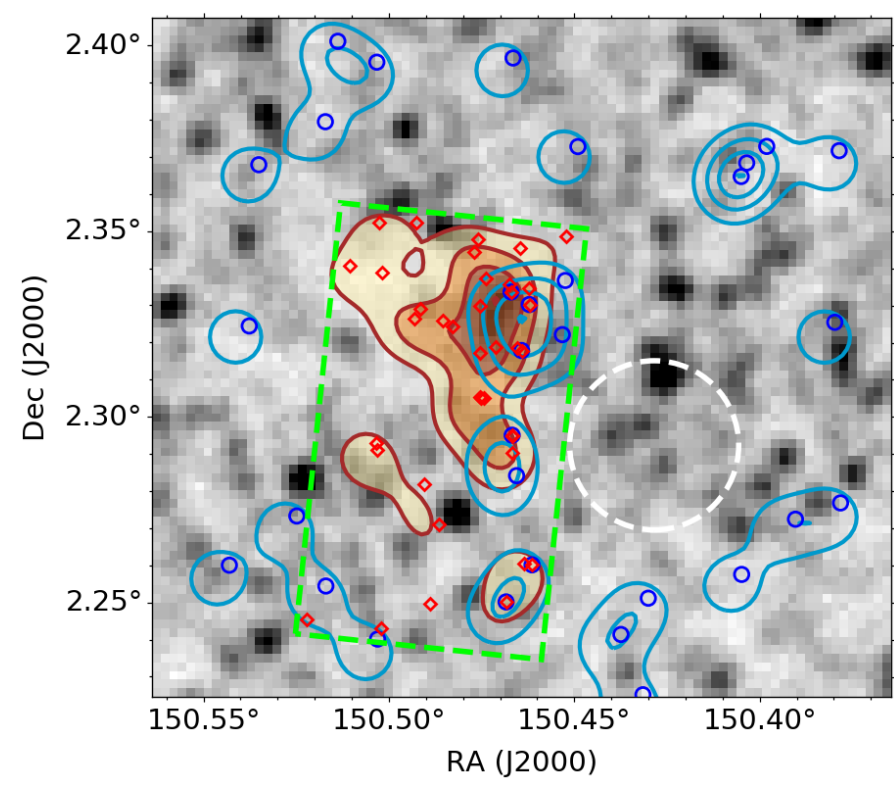

Fig. 3. Spatial distribution of spectroscopic sources (blue circles), and HAEs (red diamonds) overplotted on the SPIRE $350 \mu \mathrm{m}$ image of the G237 field. The yellow-brown filled contours show the HAE projected density, and the turquoise contours the projected density of the spectroscopic sources. The density contour levels are shown at 25, 50, 75, and $100 \%$ of the maximum value. The dashed green rectangle represents the MOIRCS field of view where the HAE were selected (Koyama et al. 2021). The spectroscopic and HAE density peaks are $38^{\prime \prime}$ apart. The white circle represents a masked region in the L16 catalog due to the presence of a bright star.

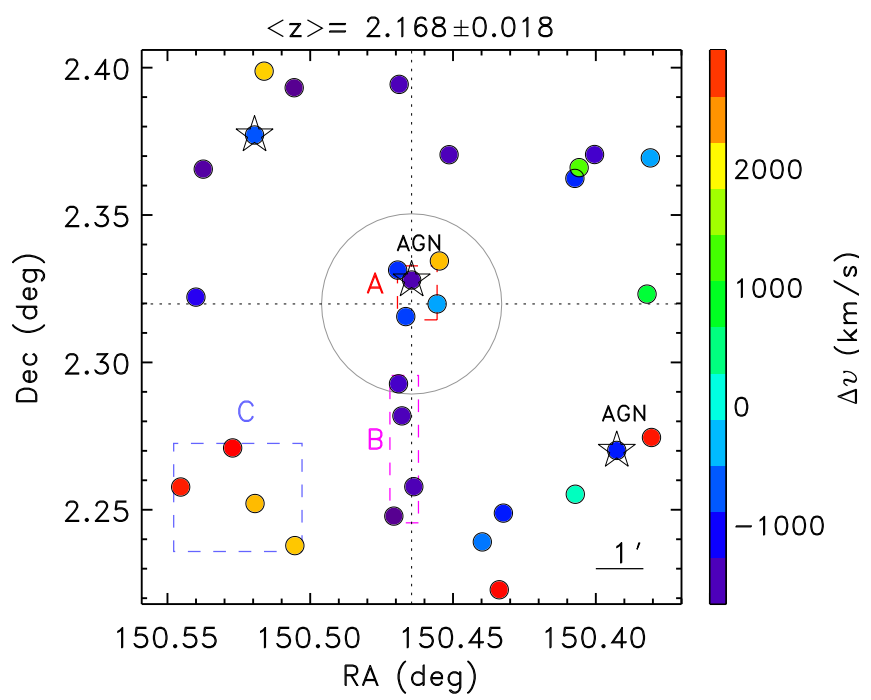

Fig. 4. Spatial distribution of sources with $z=2.15-2.20$ in the field of PHz G237.01+42.50. Different colors correspond to the velocity offset from the annotated mean redshift as indicated by the color bar. AGN are sources spectroscopically classified AGN, and black stars are $\mathrm{X}$-ray luminous AGN. A gray circle with radius corresponding to $2 \mathrm{Mpc}$ comoving at the annotated mean redshift and centered on the structure center is shown. Dashed rectangles delineate the A, B, and C components described in Sect. 3.4, and shown in Fig. 6.

Fig. 5). Assuming as reference redshift, the mean value (i.e., $\langle z\rangle=2.168 \pm 0.018)$, the 31 sources can be split in two substructures with either high $\left(v \sim 2000-3000 \mathrm{~km} \mathrm{~s}^{-1}\right)$ or low ( $v$ from -1700 to $0 \mathrm{~km} \mathrm{~s}^{-1}$ ) velocities. Based on the sources redshift and

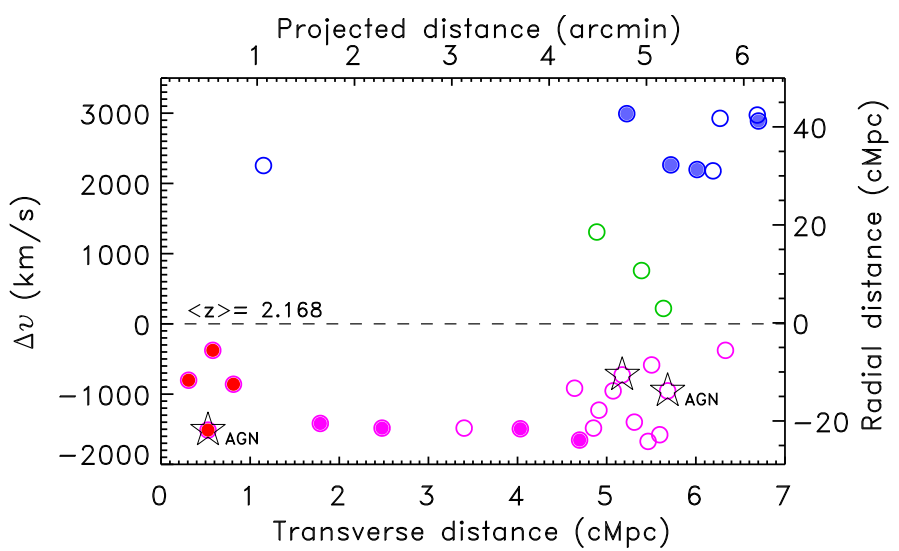

Fig. 5. Velocity offset or radial distance from the mean structure redshift $(\langle z\rangle=2.168$; dashed line) as a function of projected distance from the structure center (defined by the coordinates median). Filled circles represent sources in the 3 components described in Sect. 3.4: A in red, $\mathrm{B}$ in magenta, and $\mathrm{D}$ in blue. The remaining members are shown as open circles (magenta if at $2.15 \leq z<2.164$, blue if at $z>2.19$, and green if at intermediate redshifts, $2.164<z<2.19$ ). Spectroscopically identified AGN are annotated, and X-ray selected AGN are marked with black stars.

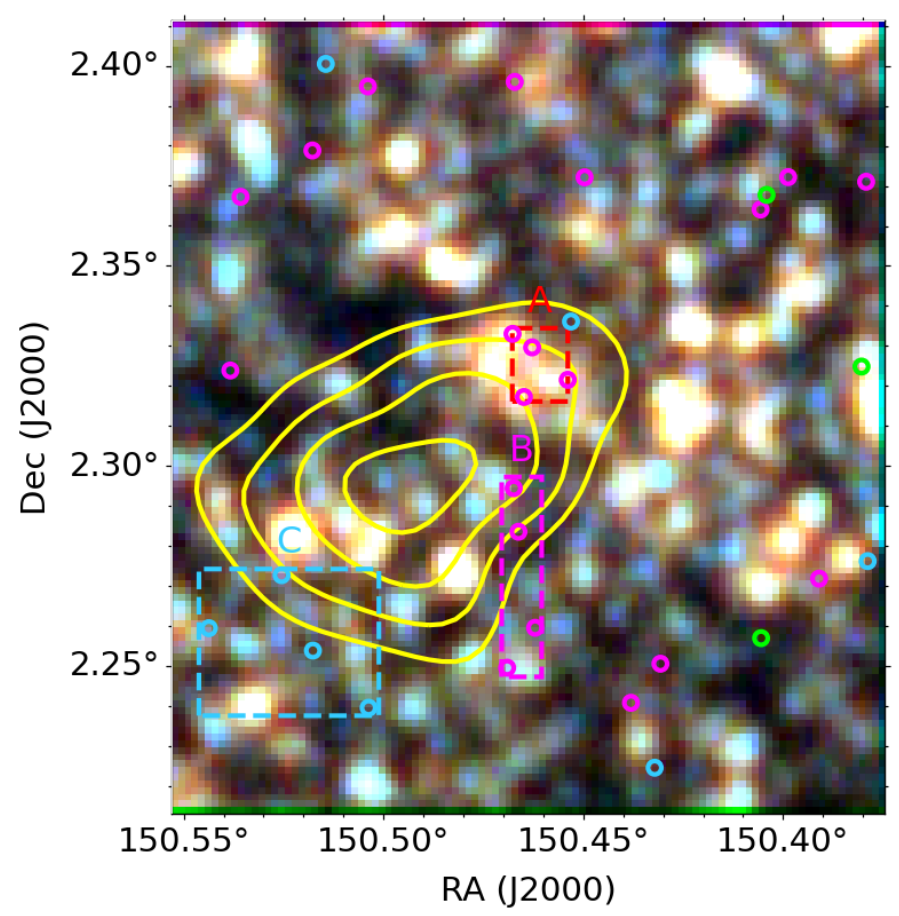

Fig. 6. Three-color Herschel/SPIRE (R: $500 \mu \mathrm{m}, \mathrm{G}: 350 \mu \mathrm{m}, \mathrm{B}: 250 \mu \mathrm{m})$ $10^{\prime} \times 12^{\prime}$ image of the G237 field. The yellow contours represent the Planck red-excess signal at $50,62.5,75$ and $87.5 \%$ of the peak value. Spectroscopic members are shown as open circles (magenta: $2.15 \leq$ $z<2.164$, green: $2.164<z<2.19$, and light blue: $2.19<z<2.20$ ). The regions A, B, and C, enclosing the groups of sources described in Sect. 3.4, are shown as dashed rectangles (red and magenta for those at $2.15 \leq z<2.164$, light blue for those at $2.19<z<2.20$ ).

projected spatial distribution, we identify three components, as illustrated in Fig. 6.

We first consider the group of sources with negative velocity offsets, corresponding to 20 sources with redshift $2.15 \leq z<$ 2.164. Within this group, we distinguish a core (A component; $0.8^{\prime} \times 1.1^{\prime}$ region; dashed red rectangle in Fig. 6) containing four 

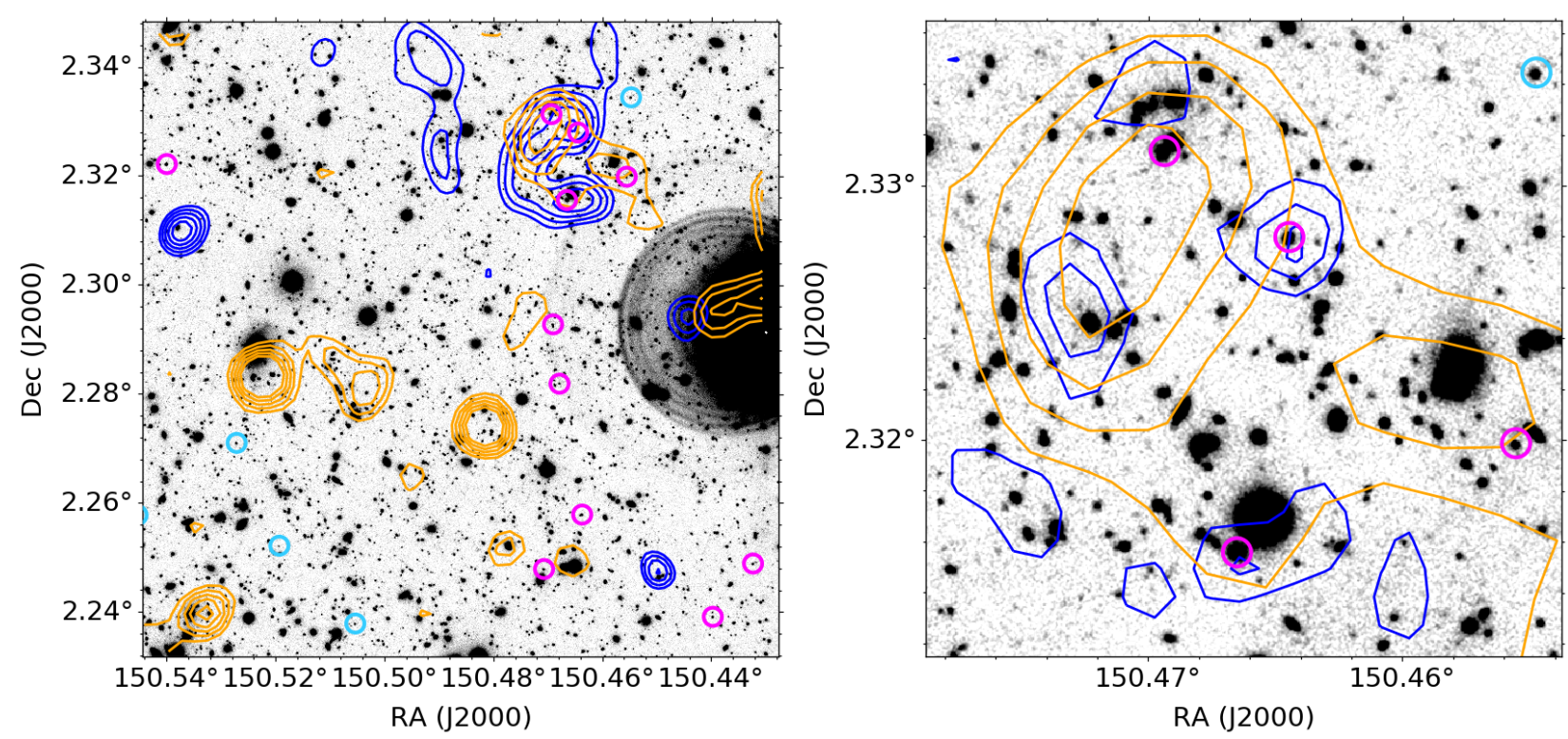

Fig. 7. UltraVISTA $K_{s}$-band image of G237. The spectroscopic members in G237 are shown as open circles (magenta: $2.15 \leq z<2.164$, and light blue: $2.19<z<2.20$ ). SPIRE $350 \mu \mathrm{m}$ contours are shown in orange, and XMM $4.5-10 \mathrm{KeV}$ contours in blue. Left panel: size of $7^{\prime} \times 7^{\prime}$, and right panel: zoom of $1.5^{\prime} \times 1.5^{\prime}$ where the largest densities of spectroscopic members and of X-ray and sub-mm sources are situated.

sources (see red full circles in Fig. 5). This region exhibits the highest source density (4.4 sources $\left.\operatorname{arcmin}^{-2}\right)$. In the same redshift group, there are four sources at similar redshifts $(\Delta v=$ $\left.238 \mathrm{~km} \mathrm{~s}^{-1}\right)$, and located in an elongated region $\left(0.6^{\prime} \times 3^{\prime}\right.$; region B) with velocity offsets increasing with the distance from the core. These four sources, and one in the core, ID SL03, are all aligned south of the core, and their velocity offsets are consistent with them falling and accelerating into the core. The remaining 12 sources are more spread out and located in an annulus between $3^{\prime}$, and $6.5^{\prime}$ from the core. The group of 20 spectroscopic members with $2.15 \leq z<2.164$ occupies a volume of $9.5^{\prime} \times 9.3^{\prime} \times 1302 \mathrm{~km} \mathrm{~s}^{-1}$, corresponding to a comoving volume of $\sim 15.29 \times 14.96 \times 19.1 \mathrm{cMpc}^{3}$, or $4376 \mathrm{cMpc}^{3}$. We note that this volume estimate is not corrected for peculiar velocities (see Sect. 6.1). Among the remaining 11 sources with positive velocity offsets, eight are at $2.19<z<2.20$, corresponding to $\Delta v=788 \mathrm{~km} \mathrm{~s}^{-1}$. Four sources in this group are in a $2.7^{\prime} \times 2.2^{\prime}$ region [C component].

There are thus in total 31 sources with $2.15 \leq z<2.20$, corresponding to $\Delta v=4646 \mathrm{~km} \mathrm{~s}^{-1}$ over a $\sim 10^{\prime} \times 11^{\prime}$ region. The volume occupied by all 31 spectroscopic members is $\sim 16 \times 17 \times 67 \mathrm{cMpc}^{3}$, or $18532 \mathrm{cMpc}^{3}$.

Based on the spectroscopic sources spatial distribution, we test the hypothesis that the full sample, and the two groups at $z \simeq 2.15-2.164$, and 2.19-2.20 might be structures. There is no clear and consensual definition of a high- $z$ structure. As a reference we consider the criterion used by Eisenhardt et al. (2008) valid for $z>1$ clusters for which at least five members must be located within a radius of $2 \mathrm{pMpc}$ and have spectroscopic redshifts within $\pm 2000(1+\langle z\rangle)$, corresponding to $4^{\prime}$, and $\simeq \pm 6300 \mathrm{~km} \mathrm{~s}^{-1}$ at the redshift of $\mathrm{G} 237$. The group at $2.15 \leq z<$ 2.164 has 11 sources within 2 pMpc, and that at $2.19<z<2.20$ has five sources within $2 \mathrm{pMpc}$, thus both satisfy this criterion. If we consider the full list of 31 spectroscopic sources, 16 of which are located within a $2 \mathrm{pMpc}$ radius and satisfy the velocity criterion, we can thus consider the full sample and the two smaller groups as being structures. In the following, we refer to the group at redshifts $2.15 \leq z<2.164$ as substructure 1 (ss1), that at redshifts $2.19 \leq z<2.20$ as substructure 2 (ss2), and to all 31 sources as the full structure. In Sect. 6.1 we analyze whether each of these structures can be considered a protocluster, based on the redshift at which they will collapse and on the estimated $z=0$ halo mass. We define as center of the full structure, the median coordinates of all 31 members, $\alpha=150.46449 \mathrm{deg}$, and $\delta=2.3198330 \mathrm{deg}$.

\section{Sub-mm emission in G237}

\subsection{Planck versus Herschel}

One of the main questions regarding the $\mathrm{PHz}$ sources is the origin of the sub-mm signal detected by Planck. SPIRE observations provide images at similar wavelengths as Planck, but with a better sensitivity and a spatial resolution 10 times higher (the SPIRE FWHM at $350 \mu \mathrm{m}$ being $25^{\prime \prime}$ ). In the following, we compare the Planck signal in G237, with the total flux density from all SPIRE sources detected at $350 \mu \mathrm{m}$, and $500 \mu \mathrm{m}$, respectively. The SPIRE observations in the Cosmos field are from the HerMES survey (Oliver et al. 2012). The SPIRE $5 \sigma$ sensitivity limits in the field are 8.0,6.6, and $9.5 \mathrm{mJy}$, at 250, 350, and $500 \mu \mathrm{m}$, respectively.

We compare Planck and SPIRE flux densities in the region where the signal in the RX map is higher than $50 \%$ of the maximum value (hereafter $\mathrm{PHz}$ area). This region, shown with yellow contours in Fig. 6 overlaid on the SPIRE image of the G237 field, has an area of $29.5 \mathrm{arcmin}^{2}$. To compute the SPIRE flux density we consider all SPIRE sources within the region drawn from the band-merged (or cross-ID, hereafter xID) DR4 catalog extracted on Starfinder blind $250 \mu \mathrm{m}$ positions (Roseboom et al. 2010; Wang et al. 2014). All selected sources are detected at more than $3 \sigma$ in at least one of the three SPIRE bands, where $\sigma$ includes both the instrumental and confusion error ${ }^{7}$. There are 46 SPIRE sources in the $\mathrm{PHz}$ area, of which 14 are detected at more than $3 \sigma$ at $350 \mu \mathrm{m}$, and nine at $500 \mu \mathrm{m}$. The total SPIRE flux

\footnotetext{
7 The confusion noise is estimated to be 5.8, 6.3 and $6.8 \mathrm{mJy}^{-1}$ beam $^{-1}$ at 250, 350 and $500 \mu \mathrm{m}$, respectively (Nguyen et al. 2010).
} 
Table 1. Number of SPIRE sources and total flux density in the G237 PHz area.

\begin{tabular}{|c|c|c|c|c|c|c|c|c|}
\hline \multicolumn{4}{|c|}{ N SPIRE in PHz area ${ }^{(a)}$} & \multirow{2}{*}{$\begin{array}{c}\text { PHz area } \\
(b) \\
\left(\operatorname{arcmin}^{2}\right)\end{array}$} & \multirow{2}{*}{$\begin{array}{c}{\text { Planck } S_{350}(c)}_{(\mathrm{mJy})} \\
\end{array}$} & \multirow{2}{*}{$\begin{array}{c}\operatorname{SPIRE} \sum S_{350}{ }_{(\mathrm{mJy})}^{(d)} \\
\end{array}$} & \multirow{2}{*}{$\begin{array}{c}{\text { Planck } S_{550}(c)}_{(\mathrm{mJy})} \\
{ }^{2}\end{array}$} & \multirow{2}{*}{$\underset{(\mathrm{mJy})}{\operatorname{SPIRE} \sum S_{500}(d)}$} \\
\hline $\mathrm{xID}$ & $350 \mu \mathrm{m}$ & $500 \mu \mathrm{m}$ & red & & & & & \\
\hline 46 & 14 & 9 & 13 & 29.5 & $518 \pm 42$ & $722 \pm 45$ & $349 \pm 34$ & $404 \pm 35$ \\
\hline
\end{tabular}

Notes. ${ }^{(a)}$ The number of $\mathrm{xID}, 350 \mu \mathrm{m}$, and $500 \mu \mathrm{m}$ sources in the PHz designate the number of SPIRE sources with at least one $3 \sigma$ detection in any band, at $350 \mu \mathrm{m}$, or at $500 \mu \mathrm{m}$, respectively. The red sources are the xID sources with red sub-mm colors (i.e., $S_{350 \mu \mathrm{m}} / S_{250 \mu \mathrm{m}}>0.7$ and $S_{500 \mu \mathrm{m}} / S_{350 \mu \mathrm{m}} \geq 0.6$ ). ${ }^{(b)}$ The PHz area is defined as all adjacent pixels with a value above $50 \%$ of the maximum value in the RX map (see text). ${ }^{(c)}$ The Planck flux densities, Planck $S_{\lambda}$, have been computed by adding the flux density values of all pixels within the PHz area. ${ }^{(d)}$ The SPIRE flux densities have been computed by adding the flux density of all xID sources within the same region.

density at 350 and $500 \mu \mathrm{m}$ is the total flux density obtained from all detected SPIRE sources located in the PHz area. The Planck flux density at $350 \mu \mathrm{m}$, and $550 \mu \mathrm{m}$ is instead obtained by adding up the signal from all the pixels in the cleaned maps within the $\mathrm{PHz}$ area. The measured total SPIRE and Planck flux densities are reported in Table 1. We note that the Planck flux densities in the $\mathrm{PHz}$ area correspond to about 75 , and $60 \%$ of the total flux densities at, respectively, 350, and $550 \mu \mathrm{m}$ listed in the $\mathrm{PHz}$ catalog (Planck Collaboration Int. XXXIX 2016), and reported in Sect. 3. In comparing SPIRE and Planck flux densities, we need to take into account the difference in the filter transmission curves. Based on the average template derived from the ALESS sample (da Cunha et al. 2015), we expect $S_{350 \mu \mathrm{m}}^{\text {SPIRE }} / S_{350 \mu \mathrm{m}}^{\text {Planck }}=0.84$ 0.96 , and $S_{500 \mu \mathrm{m}}^{\text {SPIRE }} / S_{550 \mu \mathrm{m}}^{\text {Plank }}=1.01-1.0$ at $z=0.5-3.0$.

The SPIRE and Planck flux densities at $500 \mu \mathrm{m}$ are consistent within $1 \sigma$, while the SPIRE $350 \mu$ m flux density is $>3 \sigma$ larger than the Planck $350 \mu \mathrm{m}$ flux density. This comparison indicates that the SPIRE observations are deep enough to detect all sources that contribute to the Planck flux density (Herranz et al. 2013; Clements et al. 2014), and that the difference at $350 \mu \mathrm{m}$ might be due to the signal from low- $z$ galaxies that were removed in the Planck cleaned images (see details on the cleaning procedure in Planck Collaboration Int. XXXIX 2016).

\subsection{Sub-mm source density}

Here, we investigate whether G237 is overdense of red SPIRE sources, as found for the majority of $\mathrm{PHz}$ sources (Planck Collaboration Int. XXVII 2015). To this end, we consider all red SPIRE sources over a $1^{\circ} \times 1^{\circ}$ region detected at $>1 \sigma$ in all three SPIRE bands, and at $>3 \sigma$ in at least one band where $\sigma$ includes the instrumental and confusion noises. For each source we compute a flux-weighted local density given by the distance distribution to the nearest 10th neighbors as:

$\delta_{i}=\frac{W_{i}}{\pi d_{i, 10}^{2}} \sum_{j=1}^{10} \exp \left[-0.5\left(\frac{d_{i, j}}{d_{i, 10}}\right)^{2}\right]$,

where $d_{i j}$ is the distance to the $j$ th source, $d_{i, 10}$ is the distance from the $i$ th source to the 10th nearest neighbor, and $W_{i}$ is the $i$ th source weighting factor. This factor is the ratio between the $i$ th source flux density minus the minimum flux density in the field, and the difference between the maximum and the minimum flux densities, where all flux densities are at $350 \mu \mathrm{m}$ (adapted from Clements et al. 2014). From the distribution of $\delta_{i}$ values, we then compute a mean background density $\left(\rho_{\text {bck }}\right)$ as the $3 \sigma$ clipped mean and an associated uncertainty given by the rms $\left(\mathrm{rms}_{\mathrm{bck}}\right)$. Based on the positions of the red SPIRE sources, and

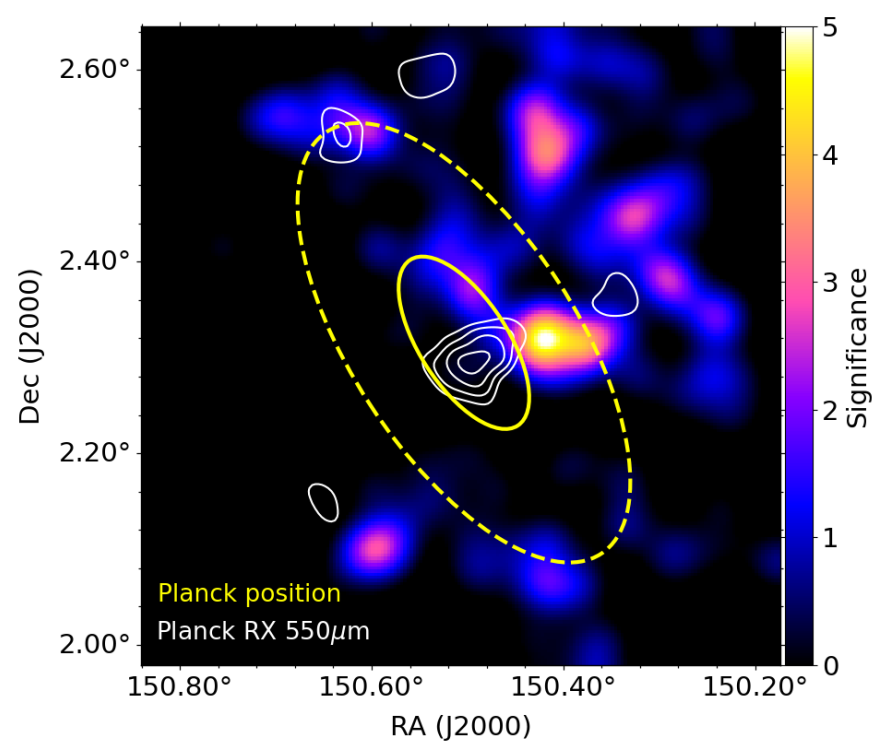

Fig. 8. $40^{\prime} \times 40^{\prime}$ map centered on G237 showing the significance of the flux-weighted density contrast of the red SPIRE sources based on the distance distribution to the 10th nearest neighbors. White contours represent the Planck signal in the $550 \mu \mathrm{m}$ red-excess map at 50, 62.5, 75, and $87.5 \%$ of the peak value. The yellow ellipse represents the double Gaussian fit (solid: full axis equal to FWHM, dashed: half axis equal to $3 \sigma^{\text {pos }}$ ) obtained when measuring the position and extent of G237 in the Planck maps. The map and the contours have been smoothed with a $3^{\prime}$ Gaussian filter.

on their $\delta_{i}$ values, we generate a two-dimensional density map in a $40^{\prime} \times 40^{\prime}$ region centered on G237 after convolution with a Gaussian kernel with a FWHM of $3^{\prime}$. From the density map we derive a density contrast map, and a density significance map (see definition in footnote ${ }^{6}$ ). The overdensity significance map of the red SPIRE sources, shown in Fig. 8, has a peak at $\gtrsim 5 \sigma$ centered at $\alpha=150.4186^{\circ}, \delta=+2.3206^{\circ}$. In Table 2, we list the maximum density contrast and significance values situated within $3 \sigma^{\text {pos }}$ from the Planck position (see dashed yellow ellipse in Fig. 8), and the corresponding coordinates. We also list the area of contiguous pixels at $>3 \sigma$ significance around the maximum in the density significance map. This overdensity peak is at $\sim 5$ arcmin from the Planck position, which corresponds to $\sim 1-2 \sigma^{\text {pos }}$ considering the source extension, which is given by an ellipse with major and minor axis with $\sigma^{\text {pos }}$ of $5.3 \pm 0.4^{\prime}$, and $2.3 \pm 0.2^{\prime}$, respectively (see yellow ellipses in Fig. 8).

We note that this method differs from that used in Planck Collaboration Int. XXVII (2015) to characterize the red SPIRE source overdensity in the $\mathrm{PHz}$ sample. First, they measured the overdensity only of the red SPIRE sources within the $\mathrm{PHz}$ area (or Planck IN region; see previous section), and the mean density 
Table 2. Red SPIRE overdensity parameters.

\begin{tabular}{|c|c|c|c|c|}
\hline \multicolumn{2}{|c|}{ Maximum } & \multirow{2}{*}{$\begin{array}{c}\text { Area }(>3 \sigma) \\
\left(\operatorname{arcmin}^{2}\right)\end{array}$} & \multirow{2}{*}{$\begin{array}{l}\alpha_{\max } \\
(\mathrm{deg})\end{array}$} & \multirow{2}{*}{$\begin{array}{l}\delta_{\max } \\
(\operatorname{deg})\end{array}$} \\
\hline$\delta$ & Signif. & & & \\
\hline 10.4 & 5.1 & 12.8 & 150.4186 & 2.3206 \\
\hline
\end{tabular}

was measured in $\sim 20^{\prime} \times 20^{\prime}$ images after excluding the $\mathrm{PHz}$ area. Finally, the uncertainty associated with the mean density field used to compute the overdensity significance was the standard deviation, rather than the rms. In G237, this method would not yield a significant overdensity because the overdensity is situated just outside the $\mathrm{PHz}$ area. An offset between the red SPIRE source overdensity peak and the $\mathrm{PHz}$ area, although not common, is observed in several PHz sources. Such an offset is, however, not surprising as the Planck red-excess signal and the SPIRE red sources do not trace exactly the same information. We note that the Planck source position is the result of a double Gaussian fit on the $550 \mu \mathrm{m}$ cleaned map and not on the red-excess one, and $\mathrm{G} 237$ is among the most extended $\mathrm{PHz}$, with only $18 \%$ of all $\mathrm{PHz}$ having a major axis with $F W H M>$ $12.4^{\prime}$, as measured in G237. Considering the source extension, the Planck position and the overdensity peak are consistent.

In the next section, we analyze the spatial distribution and properties of the SPIRE sources in the field to understand the nature of such an overdensity, and its possible association with the spectroscopic structure at $z \sim 2.16$.

\subsection{SPIRE member candidates}

Here, we consider all the SPIRE sources in G237 (the $10^{\prime} \times 11^{\prime}$ region shown in Fig. 4) with at least $\mathrm{a} \geq 3 \sigma$ detection and examine the available ancillary data to assess whether any of them might be associated with the spectroscopic sources. To this end, we take advantage of the available deep VLA $3 \mathrm{GHz}$, and MIPS[24 um] data (Smolčić et al. 2017; Sanders et al. 2007), to identify the most likely counterpart to each SPIRE source. We can indeed assume that a source detected in the radio at $3 \mathrm{GHz}$, or at $24 \mu \mathrm{m}$ is the most likely counterpart to the SPIRE source as the emission at these wavelengths and the sub-mm radiation are mainly powered by star formation and, thus, highly correlated (de Jong et al. 1985; Wunderlich et al. 1987; Sopp \& Alexander 1991; Rieke et al. 2009).

There are 187 SPIRE sources, of which 183 have at least one VLA $3 \mathrm{GHz}$ or MIPS[24 $\mu \mathrm{m}$ ] counterpart within 11 arcsec, 94 with a unique VLA+MIPS counterpart, eight with 2, and one with 3 , and 18 have a unique VLA counterpart with no MIPS[24 $\mu \mathrm{m}]$ match. Out of the remaining 62 SPIRE sources with no VLA counterpart, 43 have a unique MIPS counterpart, 15 have two, and four have three MIPS matches. Thus, $155 / 183(85 \%)$ sources have a single counterpart at $3 \mathrm{GHz}$, $24 \mu \mathrm{m}$, or in both bands. The remaining 28 are matched with 61 VLA/MIPS[24 $\mu \mathrm{m}]$ sources. There are only four SPIRE sources without a match in either bands, but they are close to the region borders, and have faint SPIRE flux densities ( $\leq 20 \mathrm{mJy})$. We do not consider these sources in the following as their contribution to the Planck flux density would be low, and their association with the protocluster unlikely. Finding VLA or MIPS counterparts allows us to identify the matched source in the L16 catalog. In addition, for the MIPS-detected sources, deblended SPIRE flux densities are available. To find sources that might

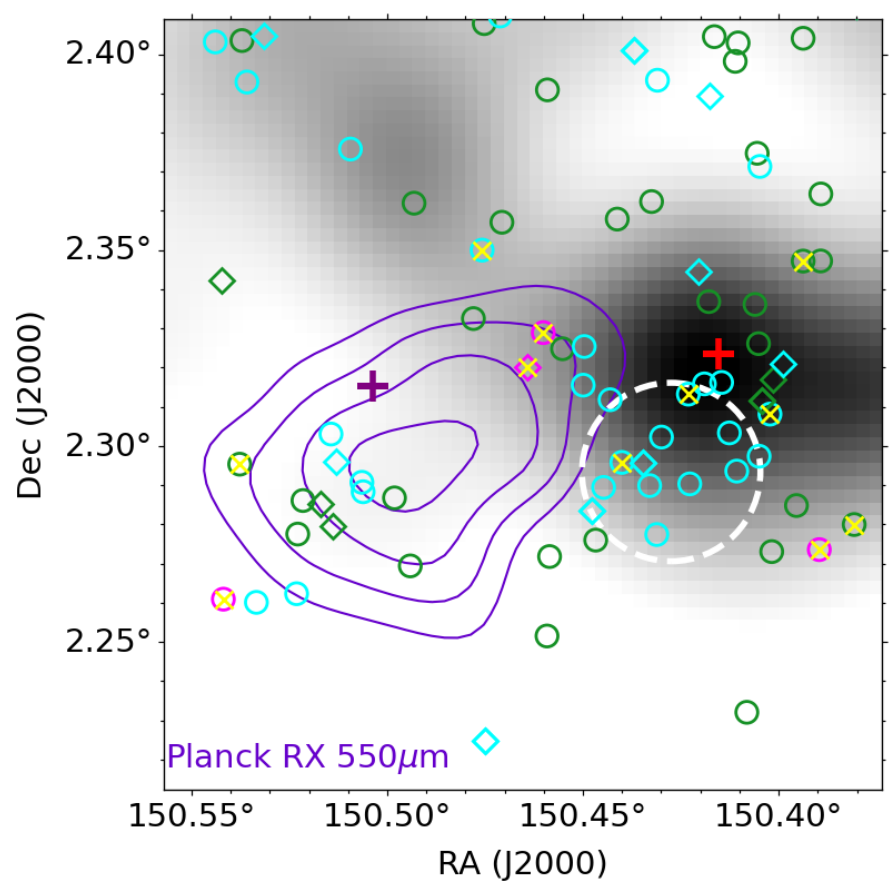

Fig. 9. Density map of red SPIRE sources, with the Planck red-excess contours overlaid in purple (at 50, 62.5, 75, 87.5\% of the highest value). The dashed white circle represents the $1.4^{\prime}$ radius circle masked in the L16 Cosmos catalog due to a bright star. The Planck position is marked with a thick purple plus sign, while the position where the density of red SPIRE sources peaks is marked with a thick red plus sign. The other symbols represent 86 SPIRE sources with colors and redshifts consistent with $z \sim 2.16$ (circles are sources with a unique VLA and/or MIPS[24 $\mu \mathrm{m}]$ counterpart, diamonds are sources with multiple VLA counterparts): magenta if $2.15 \leq z_{\text {spec }}<2.20$ (4); green if $z_{\text {phot }}>1.4$ and red (40), cyan if no redshift is available and red (42). We note that about $17(40 \%)$ of the sources with no redshift are likely at $z<1.4$; see text for more details. A yellow cross is overplotted on sources with $2.25^{\circ}<\delta<2.35^{\circ}$ and with $z_{\text {spec }}$ or with a redshift estimate between 1.8 and 2.5 (11 sources). Such a redshift estimate was obtained by requiring the observed radio flux to be reproduced assuming the radio-FIR correlation.

be members of the $z \sim 2.16$ structure out of all potential SPIRE counterparts, we select those with $2.15 \leq z_{\text {spec }}<2.20$ (4 sources; see Table 3), those with $z_{\text {phot }}>1.4$ and with sub-mm colors consistent with being red (40 sources), and those with no redshift information and red sub-mm colors (42 sources). We remove 78 sources with spectroscopic redshifts outside the redshift range of $2.15 \leq z_{\text {spec }}<2.20$, five because they are not red even if $z_{\text {phot }}>1.4$, five because they are not red and no redshift is available, and 42 with $z_{\text {phot }}<1.4$. The spatial distribution of the selected 86 SPIRE sources is shown in Fig. 9. These SPIRE member candidates clump in the region where there is a significant overdensity of red sources (see Sect. 4.2 and Table 2). In this region there is a bright star that prevents us from detecting any source in the optical and NIR, and thus none of those SPIRE sources has a redshift estimate (see cyan symbols in Fig. 9). To take into account the lack of redshift estimate due to the masked region, we apply a statistical correction to these 42 SPIRE sources without redshift. Based on the fraction of SPIRE sources in a large $\left(1.16^{\circ} \times 1.23^{\circ}\right)$ region of the COSMOS field with Herschel, MIPS, and VLA coverage, we find that $\sim 40 \%$ of counterparts of red SPIRE sources have a photometric or spectroscopic redshift $<1.4$. We can thus assume that 17 out of those 42 SPIRE sources might be at redshift $<1.4$. There are thus 
69 SPIRE counterparts in the G237 field with redshift consistent with being related to the structure.

Assuming a redshift of 2.16, we estimate the total $(8-1000 \mu \mathrm{m})$ IR luminosities $\left(L_{\mathrm{IR}}\right)$ of the SPIRE member candidates by fitting their sub-mm spectral energy distribution (SED) with a single-temperature modified blackbody model. Fits were performed using the cmcirsed package (Casey 2012), and assuming a dust emissivity-index $\beta$ equal to 1.8 . From the IR luminosities, SFR estimates are derived assuming the relationship in Kennicutt (1998), modified for a Chabrier (2003) IMF (Chabrier 2003), SFR $\left[M_{\odot} \mathrm{yr}^{-1}\right]=9.5 \times 10^{-11} L_{\mathrm{IR}}\left[L_{\odot}\right]$. The total SFR, obtained by summing the contribution from all of the 69 sources, is $14140_{-540}^{+670} M_{\odot} \mathrm{yr}^{-1}$, consistent with the Planck estimate (i.e., Planck SFR $=10173 M_{\odot} \mathrm{yr}^{-1}$; see Sect. 3). If we limit the sample to the sources with $2.25^{\circ}<\delta<2.35^{\circ}$, where the Planck signal peaks and the overdensity is located, we obtain 44 sources and a total SFR of $9232_{-468}^{+579} M_{\odot} \mathrm{yr}^{-1}$. We note that to obtain these estimates, we used as total SFR of the sources with no redshift, the value obtained by averaging the total SFR obtained in 100 subsamples containing only $60 \%$ of the sources randomly selected and without repetitions. Thus, the Planck flux density and derived total SFR can be reproduced from the SPIRE sources in the field if we select the red ones and assume a redshift of $\sim 2.2$, as implied by the sub-mm colors. However, it is highly unlikely that all these sources belong to the same structure.

To check the estimated SFRs, we compare the observed $3 \mathrm{GHz}$ fluxes with those derived assuming the radio-FIR relation obtained using Eqs. (5), and (6) in Magnelli et al. (2015) (i.e., $\left.q_{\mathrm{IR}}=(2.35 \pm 0.8) \times(1+z)^{-0.12 \pm 0.04}\right)$, and assuming a radio spectrum with a power-law index of -0.8 . We first checked that the radio fluxes predicted by this relation were consistent with the observed ones using the subsample of SPIRE sources with a radio counterpart and a spectroscopic redshift (37 sources). We then derived the redshift that yielded a radio flux consistent with the observed one, or with the $5 \sigma$ radio upper limit in case of non detection, for the 86 selected SPIRE candidate members. The derived redshifts cover a broad range with median value of $1.4 \pm 0.7$. If we restrict the considered region to $2.25^{\circ}<\delta<2.35^{\circ}$ (56 sources), and select only the SPIRE sources with an estimated redshift, as derived from the radio flux and the radioFIR relation, between 1.8 and 2.5, we find only 11 sources (see yellow crosses in Fig. 9). The total SFR associated with these 11 sources is $\sim 2410_{-110}^{+135} M_{\odot} \mathrm{yr}^{-1}$. This analysis implies that a significant fraction $(\gtrsim 62 \%)$ of the selected red SPIRE sources might be at $z<2.15, \sim 18 \%$ in the background, and only a dozen $(\lesssim 20 \%)$ might be associated with the structure at $z \simeq 2.16$. These results are consistent with the simulations carried out by Negrello et al. (2017) showing that a large fraction of $\mathrm{PHz}$ sources might suffer from source confusion. Nevertheless, the $\mathrm{PHz}$ fields might still contain structures at $z=1.5-3$, but with lower total SFRs than derived from the Planck signal, as it is the case for G237.

\section{Properties of the spectroscopic members}

In this section, we consider all the sources in the two redshift peaks and at intermediate redshifts as belonging to a unique structure with the components described in Sect. 3.4. We analyze their main properties, such as morphology, stellar mass, SFR, AGN activity, as a function of location within the structure. The goal, in this section, is to investigate any relationship between their properties and the environment. For this analysis we use the L16 multiwavelength catalog, and additional catalogs and images from the IRSA public repository ${ }^{8}$.

\subsection{Sub-mm properties}

PHzG237.0+42.5 was discovered by Planck because of its bright and red sub-mm emission. Spectroscopic observations in the G237 field reveal an overdensity of sources at $2.15 \leq$ $z<2.20$. The connection between the sub-mm emission and the overdensity at $z \simeq 2.16$ is likely, but it could also be due to a projection effect with a foreground overdensity providing additional flux. To investigate such a connection we examine the spectroscopic members that might be sub-mm detected. Six SPIRE sources have a spectroscopic member located within the SPIRE beam $\left(<11^{\prime \prime}\right)$, IDs SL01, SL03, 55326, 58057, 60718, and 60894. To assess whether they are the counterparts to the SPIRE sources, we examine the radio[3 GHz] and MIPS[24 $\mu \mathrm{m}$ ] images and catalogs available in the field (L16, Smolčić et al. 2017). Since the emission in the sub-mm, in the radio and in the mid-IR (MIR) are mainly powered by SF, we can assume that they are produced by the same galaxy. The higher spatial resolution in the radio and $24 \mu \mathrm{m}$ images helps to pinpoint the optical/NIR counterpart to the SPIRE source. A $24 \mu \mathrm{m}$ counterpart is found in the SPIRE beam of all 6 SPIRE sources with an associated spectroscopic member, in four cases the spectroscopic member matches the $24 \mu \mathrm{m}$ source and we can thus confirm the match between the SPIRE source and the spectroscopic galaxy.

A $3 \mathrm{GHz}$ counterpart is found in only two of the four SPIRE sources with spectroscopic member, and they both have a $24 \mu \mathrm{m}$ counterpart, but different from the spectroscopic galaxy. In three cases, a faint radio source is visible in the images, and it matches the spectroscopic galaxy, but none is present in the $3 \mathrm{GHz} 5 \sigma$ public catalog.

Based on this analysis, we assign the measured SPIRE flux to the spectroscopic IDs SL03 (SPIRE: 9741, MIPS: 192876), 58057 (SPIRE: 16974, MIPS: 677305), 55326 (SPIRE:13155, MIPS: 182700), and 60984 (SPIRE: 10299, MIPS: 631549). The SPIRE flux densities and estimated IR $(8-1000 \mu \mathrm{m})$ luminosities of these four sources are listed in Table 3. For the remaining spectroscopically confirmed galaxies we consider the measured SPIRE flux density as an upper limit.

In conclusion, four out of 31 members are detected by SPIRE and have $L(I R)>10^{12} L_{\odot}$, and three are in ss1. Assuming a volume density of $(3 \pm 2) \times 10^{-4} \mathrm{Mpc}^{-3}$ for DSFGs with similar luminosities and $1.3<z<3.6$ (Casey et al. 2014), the expected number of DSFGs in our substructure volume is $1.3 \pm 0.9$. Thus, the number of DSFGs corresponds to an overdensity contrast $\delta \mathrm{DSFG}=(3-1.3) / 1.3=1.3$, with a significance of $(3-1.3) / 0.9=1.9 \sigma$.

Overdensities of DSFGs at $z \sim 2$ are often associated with protocluster candidates (Blain et al. 2004), but confirming their membership through spectroscopic identification is a challenging process (Casey et al. 2017). In the G237 field, we carried out targeted spectroscopic observations with LBT/LUCI of five SPIRE sources, and confirmed membership of only one DSFG ( 20\%). Lacaille et al. (2019) carried out spectroscopic observations of 56 DSFGs in two protoclusters at $z \sim 2.3$, and 2.85 (HS 1700+64, and HS 1549+19, respectively). They were able to measure a spectroscopic redshift for five DSFGs (9\%), and confirm membership of four DSFGs (7\%).

\footnotetext{
8 The Cosmos spectroscopic redshift catalog is available on this page: https://irsa.ipac. caltech. edu/data/COSMOS/overview.
} html. 
Table 3. SPIRE detected spectroscopic targets.

\begin{tabular}{lrccccccc}
\hline \hline ID & $\begin{array}{r}\text { SPIRE } \\
\text { ID }\end{array}$ & $z_{\text {spec }}$ & $\begin{array}{c}S_{24 \mu \mathrm{m}} \\
(\mu \mathrm{Jy})\end{array}$ & $\begin{array}{c}S_{250 \mu \mathrm{m}} \\
(\mathrm{mJy})\end{array}$ & $\begin{array}{c}S_{350 \mu \mathrm{m}} \\
(\mathrm{mJy})\end{array}$ & $\begin{array}{c}S_{500 \mu \mathrm{m}} \\
(\mathrm{mJy})\end{array}$ & $\log \left(L_{\mathrm{IR}} / L_{\odot}\right)$ & $\begin{array}{c}\mathrm{SFR}_{\mathrm{IR}} \\
\left(M_{\odot} \mathrm{yr}^{-1}\right)\end{array}$ \\
\hline 55326 & 13155 & 2.1576 & $172 \pm 19$ & $9.6 \pm 2.0$ & $<19.7$ & $<15.3$ & $12.12 \pm 0.02$ & $126 \pm 5$ \\
58057 & 16974 & 2.1517 & $208 \pm 15$ & $8.2 \pm 2.0$ & $<19.7$ & $<15.5$ & $12.05 \pm 0.02$ & $106 \pm 5$ \\
SL03 & 9741 & 2.1592 & $263 \pm 25$ & $12.3 \pm 2.0$ & $17.8 \pm 4.0$ & $15.2 \pm 4.3$ & $12.24 \pm 0.02$ & $165 \pm 9$ \\
60984 & 10299 & 2.1982 & $425 \pm 16$ & $16.0 \pm 2.0$ & $15.7 \pm 2.5$ & $12.1 \pm 3.6$ & $12.36 \pm 0.02$ & $216 \pm 9$ \\
\hline
\end{tabular}

On the other hand, such an association seems to be valid only for the bright DSFGs (i.e., $S_{870 \mu \mathrm{m}} \gtrsim 5 \mathrm{mJy}$; García-Vergara et al. 2020), corresponding to SPIRE flux densities $\gtrsim 20 \mathrm{mJy}$ assuming a dust temperature of $30-35 \mathrm{~K}$ at $z \sim 2$. The four spectroscopically identified SPIRE sources in G237 have SPIRE flux densities $\sim 10-18 \mathrm{mJy}$, thus lower than expected to trace massive structures. The brightest DSFG, out of the $11 \mathrm{mem}-$ ber candidates selected in Sect. 4.3, has a SPIRE flux density of $\sim 50 \mathrm{mJy}$. This DSFG is bright enough to trace a massive structure, but its membership to G237 needs to be spectroscopically confirmed.

\subsection{Members colors and classification}

To characterize the SF activity and extinction level of the selected members we examine their location within two well known diagnostic diagrams, the rest-frame $N U V-r$ versus $r-K$, and $U-V$ versus $V-J$ diagrams (Williams et al. 2009; Arnouts et al. 2013). The two diagrams are widely used in the literature to separate quiescent galaxies from SFGs up to high redshifts $(z \lesssim 5$; Lemaux et al. 2020; Shahidi et al. 2020). A galaxy position in these diagrams depends on its SFR, extinction, specific SFR (sSFR = SFR/M), age, and metallicity (for further details, see Arnouts et al. 2013; Davidzon et al. 2016).

We show the positions of the 31 spectroscopic members in these color diagrams in Fig. 10. Rest-frame colors are computed from the best fit SEDs (see Sect. 5.4). All members are located in the SFG region. The vast majority ( $87 \%$ ) clump in the region where blue SFGs are located, consistent with young stellar populations, and low extinction. The remaining eight sources (their IDs are annotated in the figure) are more scattered within the SFG region and exhibit redder colors. These eight sources include the four sources detected in the sub-mm, and four AGN (red symbols in the figures), of which two are also sub-mm detected (i.e., 55326 and 58057). The four sub-mm detected sources might be redder because of dust extinction. Regarding the four AGN, their redder colors might be due to the AGN component or to an older stellar component. Galaxies hosting an AGN might be transitioning to the quiescent region as a consequence of reduced SF activity caused by AGN feedback (see e.g. Krishnan et al. 2017; Shimakawa et al. 2018). The four AGN in the sample are, in general, redder than the SFG members in colors that include a NIR band, such as $J$ or $K$, and they are not systematically closer to the quiescent region than the SFGs (see red full circles in Fig. 10). This behavior suggests that their redder colors are more likely due to the presence of the AGN component whose contribution increases with wavelength, rather than to a change in the stellar population.

The similar UV-optical colors of the majority of our spectroscopic members allow us to carry out an analysis of their spectral properties by co-adding their spectra. In the next section, we will analyze the stacked spectrum and compare it with models to estimate the average stellar age and metallicity. The AGN will not be taken into account in the following analysis.

\subsection{Ultraviolet spectral properties}

\subsubsection{Stacked UV spectrum}

The UV spectrum of SFGs is rich of spectral features carrying information on several components within a galaxy, like the stellar population, the ISM, the ionized nebulae (see e.g., Fanelli et al. 1988; Kewley et al. 2019). Since the available spectra are noisy and of moderate resolution, we make a coadded spectrum by stacking all spectra that cover a similar restframe wavelength range and exhibit consistent properties. This selection yields 26 SFGs with zCosmos-Deep VIMOS spectra. The stacked spectrum is obtained by taking the median value after a $3 \sigma$ clipping procedure and normalizing all spectra using the average flux in two regions free of strong features, from 1580 to $1830 \AA$, and from 1925 to $2060 \AA$. The co-added spectrum is then corrected by Galactic absorption assuming $E(B-V)=0.145(\text { Schlafly \& Finkbeiner } 2011)^{9}$, and the Cardelli et al. (1989) extinction law. The stacked spectrum, shown in Fig. 11, covers a rest-frame wavelength range from 1100 to $2200 \AA$, and has a spectral resolution of $1.7 \AA$.

The stacked spectrum shows several spectral features at the expected wavelengths, providing support to the redshift estimates ( 10 out of 26 spectra have redshift flag, $z f^{10}{ }^{10}=1$, and ten have $z f g=2$, implying that about six redshift estimates might be wrong). In Appendix $\mathrm{C}$, we show that the co-added spectra obtained with all 26 non AGN at $z=2.15-2.20$, with the 16 galaxies with $z f g \geq 2$, and with the 12 galaxies at $z=2.15-2.16$, yield consistent results.

\subsubsection{UV spectral features}

In Figs. 11 and C.1 we highlight the main UV features typically observed in the spectra of SFGs: absorption lines produced in the ISM; in the photosphere of hot, young, $\mathrm{O}$ and B stars; or in stellar winds, and nebular emission lines produced in H II regions (see Kinney et al. 1993; Heckman et al. 1998; González Delgado et al. 1998; Shapley et al. 2003; Leitherer et al. 2011; Zetterlund et al. 2015; Vidal-García et al. 2017; Feltre et al. 2020). These features can potentially provide insights on the epoch and source of ICM enrichment in clusters and protoclusters (Shimakawa et al. 2015; Mantz et al. 2017), and on the star formation history of protocluster galaxies (e.g., distinguishing between continuous and bursty star formation activity; Shimakawa et al. 2014; Casey 2016; Narayanan et al. 2015).

\footnotetext{
9 https://irsa.ipac.caltech.edu/applications/DUST/

10 Based on the zCosmos-Bright sample (Lilly et al. 2007, 2009), the estimated redshifts are expected to be correct in $50 \%$ of the cases for
} $z f l g=1$, in $85 \%$ for $z f g=2$, and in $100 \%$ for $z f g=3$, and 4 . 

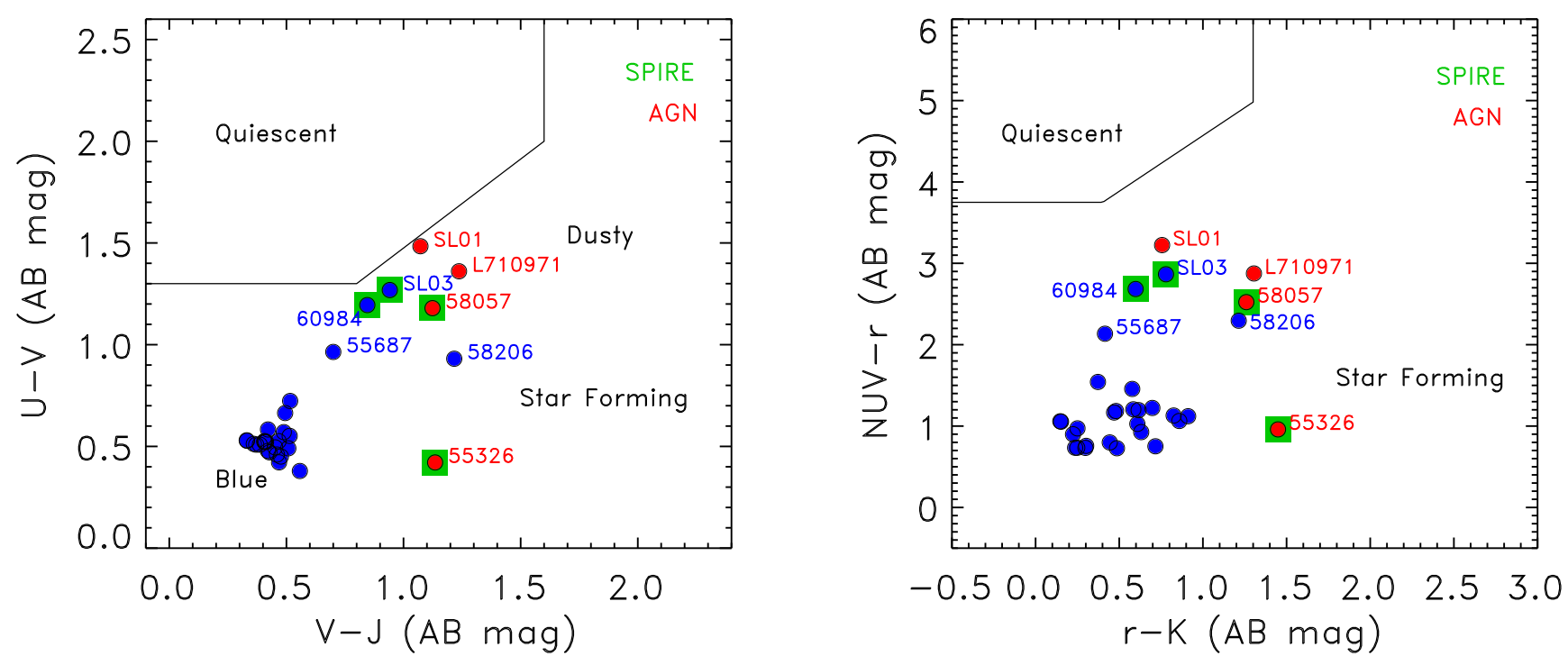

Fig. 10. Rest-frame $U-V$ vs. $V-J$ colors (left panel) and rest-frame $N U V-r$ vs. $r-K$ colors (right panel) for the 31 spectroscopic members (full blue circles; AGN are shown in red, and SPIRE detected sources with a green square). The black line separates quiescent galaxies (top left) from SFGs (bottom right) in both panels (Williams et al. 2009; Arnouts et al. 2013; Davidzon et al. 2016).

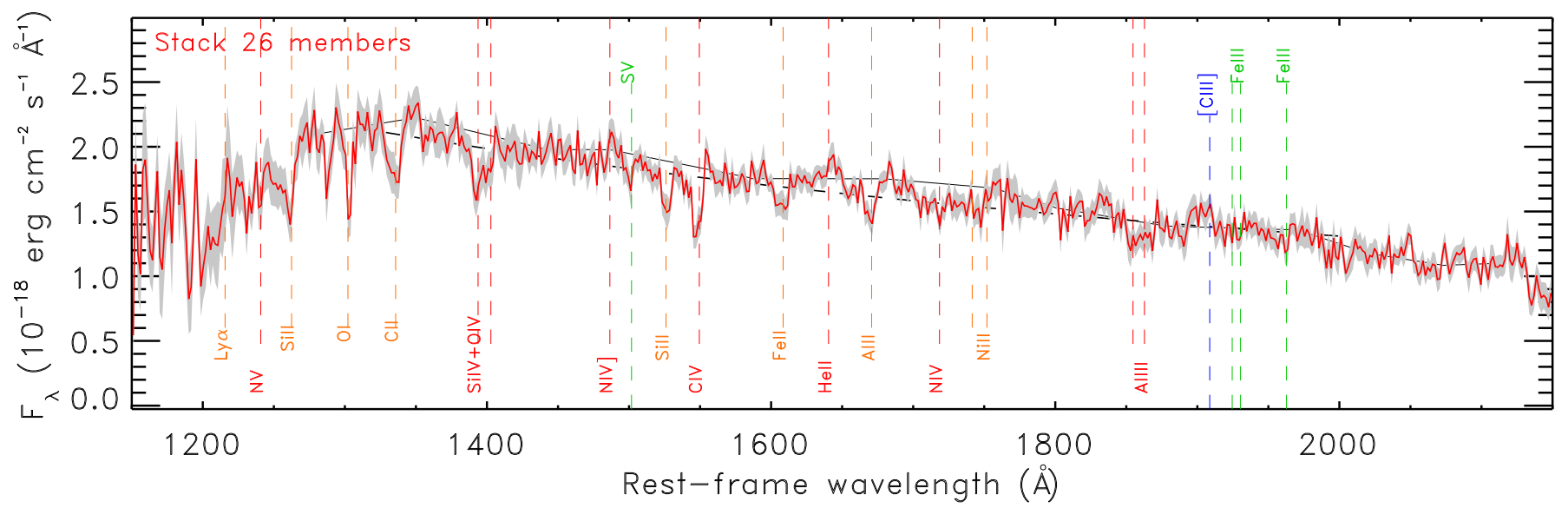

Fig. 11. Stacked rest-frame VIMOS spectrum of 26 member candidates with $z=2.15-2.20$ (red solid line). The $1 \sigma$ uncertainty is shown as gray filled region. The dashed black line is a linear fit obtained to estimate the UV spectral slope $\beta$, where $F_{\lambda} \propto \lambda^{\beta}$. The solid black curve represents the pseudo-continuum obtained by linearly interpolating the error-weighted average flux density in the "features-free" windows defined in Table 3 in Rix et al. (2004). The vertical dashed lines represent the location of the main spectral features as annotated (nebular: blue, ISM: orange, stellar photosphere: green, stellar winds: red).

In the following, we analyze the co-added spectrum to estimate the UV spectral slope, the average extinction, the intrinsic UV luminosity, and the average stellar metallicity. The UVcontinuum slope $\beta$ is obtained by fitting the UV spectrum with a power-law (i.e., $F_{\lambda} \propto \lambda^{\beta}$ ). The slope $\beta$ is sensitive to the dust reddening, the stellar age, the metal content, and even the escape fraction (Leitherer et al. 1999; Wilkins et al. 2016). For a SFG with a continuous SF and age between $10 \mathrm{Myr}$ and $100 \mathrm{Myr}$, and metallicity $Z_{*}=0.004-0.02$, the intrinsic slope $\beta$, computed over the 1230-2130 $\AA$ wavelength range, ranges from -2.7 , to -2.4 (see Fig. 71 in Leitherer et al. 1999). The $\beta$ value derived from our stack over the $1308-2000 \AA$ range is $-1.17 \pm 0.03$ (see the dashed line in Figs. 11 and C.1). Assuming that the difference between observations and model predictions is due to dust extinction and the Calzetti et al. (2000) dust prescription, the derived extinction at $1500 \AA$ would be $A_{1500}=1.84 \times \Delta \beta=2.82$, or 2.27 (corresponding to $A_{\mathrm{V}}=1.1$ 0.9 ) for intrinsic $\beta=-2.7$, or -2.4 , respectively. We note that a certain amount of dust extinction would be required even in case of a SFG with an instantaneous burst of SF. Indeed, such a model would produce an UV continuum with a slope $\beta$ that goes from $\simeq-2.6$ for an age of $10 \mathrm{Myr}$, to $\simeq-1.3$ for an age of $100 \mathrm{Myr}$, and metallicity $Z_{*}=0.004-0.02$. Assuming an extinction $A_{1500}=2.3-2.8$, the extinction-corrected UV (1500 $\AA$ ) luminosity would be $\lambda L_{1500 \AA}=(1.08 \pm 0.06) \times 10^{44} \mathrm{erg} \mathrm{s}^{-1}$. Based on the relation between the UV dust extinction and the metallicity given in Cullen et al. (2019, i.e., $\left.A_{1500}=-2.24 \times \log \left(Z_{*}\right)-2.16\right)$, we estimate an average metallicity $Z_{*}=0.006-0.011$. A more accurate estimate of the stellar metallicity is carried out in the next section.

\subsubsection{Stellar metallicity}

The stellar metallicity can be derived from the strength of photospheric absorption features, expressed in terms of indices. The relation between the various UV indices and the stellar metallicity 


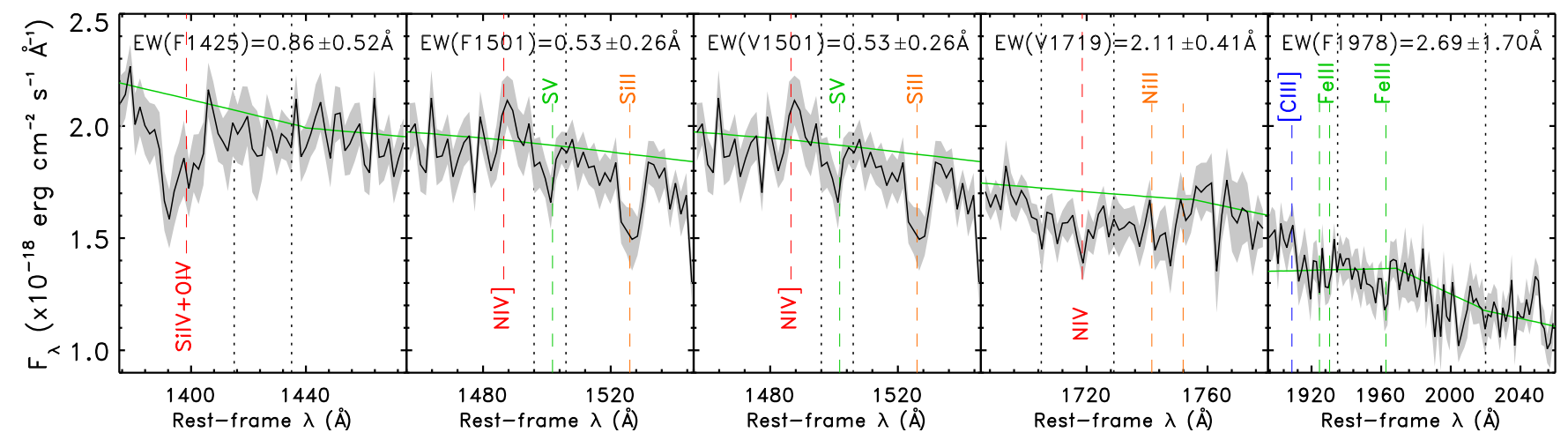

Fig. 12. Stacked spectrum (black solid line) and $1 \sigma$ uncertainty (gray filled area) and pseudo-continuum (green solid line) used to compute the indices listed in Table 4. The wavelength interval used to compute the EW is delimited by two black vertical dotted lines. The name and location of the main spectral features are indicated by dashed lines as in Fig. 11. The final EWs, computed as the median and $1 \sigma$ uncertainty measured after perturbing the spectrum 1000 times, are annotated on top of each panel.

Table 4. UV spectral indices.

\begin{tabular}{|c|c|c|c|c|}
\hline Index & $\mathrm{EW}(\AA)$ & $\log \left(Z_{*} / Z_{\odot}\right)$ & $Z_{*} / Z_{\odot}$ & Ref. ${ }^{(a)}$ \\
\hline F1425 & $0.86 \pm 0.52$ & $-0.64_{-0.82}^{+0.58}$ & $0.23_{-0.19}^{+0.64}$ & (1) \\
\hline F1501 & $0.53 \pm 0.27$ & $-0.56_{-0.86}^{+0.59}$ & $0.27_{-0.24}^{+0.78}$ & (1) \\
\hline V1501 & $0.53 \pm 0.27$ & $-0.79_{-0.46}^{+0.30}$ & $0.16_{-0.11}^{+0.18}$ & (2) \\
\hline V1719 & $2.11 \pm 0.41$ & $\begin{array}{r}-0.33_{-0.20}^{+0.46} \\
-0.18\end{array}$ & $0.47_{-0.17}^{+0.11}$ & (2) \\
\hline F1978 & $2.69 \pm 1.70$ & $-0.57_{-1.23}^{+0.69}$ & $0.27_{-0.25}^{+1.05}$ & (1) \\
\hline \multicolumn{2}{|c|}{ Error-weighted mean } & $-0.44_{-0.16}^{+0.16}$ & $0.36_{-0.11}^{+0.16}$ & \\
\hline
\end{tabular}

Notes. ${ }^{(a)}$ The relationship between the metallicity and a specific index was taken from either (1) Sommariva et al. (2012), or (2) Calabrò et al. (2021).

is derived assuming stellar models with varying star formation histories (continuous or instantaneous burst), age (typically from 5 to $150 \mathrm{Myr}$ ), IMF, and metallicity (typically $Z=0.05-$ $2 Z_{\odot}$ ) (e.g., Starburst99 models and BC03; Leitherer et al. 1999, 2011; Bruzual \& Charlot 2003). The dependence on stellar age can be usually neglected as these indices become constant after the first $\sim 30$ Myr. Several UV indices have been investigated in the literature (Rix et al. 2004; Sommariva et al. 2012; Vidal-García et al. 2017; Calabrò et al. 2021). The $1425 \AA$, and $1719 \AA$ indices are among the cleanest and least contaminated stellar metallicity tracers, and insensitive to interstellar absorption. They are thus useful to trace stellar population properties, especially at higher metallicities (Vidal-García et al. 2017). Another reliable metallicity estimator is the $1978 \AA$ index. This is associated with a blend of numerous FeIII features between $\lambda=1935 \AA$, and $2020 \AA$, and it is broader and stronger than most UV indices, and sensitive to the iron abundance. The $1978 \AA$ index is clearly visible in the spectra of starburst galaxies and strong in high metallicity environment (Heckman et al. 1998; Rix et al. 2004). The $1425 \AA$, $1719 \AA$, and $1978 \AA$ indices are the least affected by stellar age, dust, IMF, nebular continuum or interstellar absorption, they are broad and can thus be detected even at low spectral resolution (i.e., $R \sim 600$; Lilly et al. 2007, 2009), but they are shallow and require high $\mathrm{S} / \mathrm{N}$ spectra.

The most significant absorption features, well visible in the stacked UV spectrum, are the Si IV $\lambda 1397$ and the C IV $\lambda 1549$ features, respectively around 1400 , and $1550 \AA$. They both exhibit a P Cygni profile typical of high density stellar winds from giant and supergiant stars (Shapley et al. 2003; Vidal-García et al. 2017; Feltre et al. 2020). Although their strength correlates with the metallicity of the parent stars, it is difficult to use these features as metallicity indicators as they are blended with strong interstellar absorption, and affected by other processes, including the relative fractions of $\mathrm{O}$ and $\mathrm{B}$ stars (Leitherer et al. 2011).

Sommariva et al. (2012) defined a new line index, $1501 \AA$, associated with the S $5 \lambda 1501$ line, an absorption feature that originates in the photosphere of hot stars, and considered a robust metallicity indicator because almost independent of age and IMF. In addition, it is less affected by uncertainties in the continuum determination because it is defined in a small wavelength window. Based on these considerations and on the quality of our stacked spectrum, we use only the $1425 \AA, 1501 \AA$, $1719 \AA$, and $1978 \AA$ indices and the relationships between these indices and the metallicity $Z / Z_{\odot}$ derived in Sommariva et al. (2012) and Calabrò et al. (2021).

The indices are obtained by measuring the EW within well defined wavelength ranges (see Table 2 in Calabrò et al. 2021). Following Sommariva et al. (2012), Calabrò et al. (2021), we compute a pseudo-continuum by linearly fitting the errorweighted mean density fluxes computed in narrow $(\Delta \lambda \simeq$ $3 \AA$ ) windows (see Table 3 in Rix et al. 2004). Because of our low spectral resolution $(R \sim 600)$ we extended the width of such windows by $\pm 3 \AA(\Delta \lambda \simeq 9 \AA)$. The derived continuum is shown as solid black line in Fig. 11, and as a green line in Fig. 12. The EW and associated uncertainty of each index correspond to the median value, and the standard deviation obtained from 1000 computations, generated by perturbing the spectrum. The perturbation constitutes in adding noise to each wavelength element extracted from a normal distribution with a standard deviation defined by the error on the spectrum at that wavelength element. For each perturbed spectrum, an EW is measured computing a new pseudo-continuum. In Fig. 12, we show the spectrum around each index and the computed pseudo-continuum used for the EW measurement of the unperturbed spectrum. The estimated metallicities are given in Table 4.

Despite the weakness of the selected features in deriving UV indices and the uncertainty in determining the continuum, all estimates are consistent within $1 \sigma$ (see Fig. 13), and yield, on average, an error-weighted average metallicity $Z_{*} / Z_{\odot}=0.36_{-0.11}^{+0.16}$, corresponding to $Z_{*}=0.005_{-0.002}^{+0.002}$ (assuming $Z_{\odot}=0.0142 ;$ Asplund et al. 2009). 


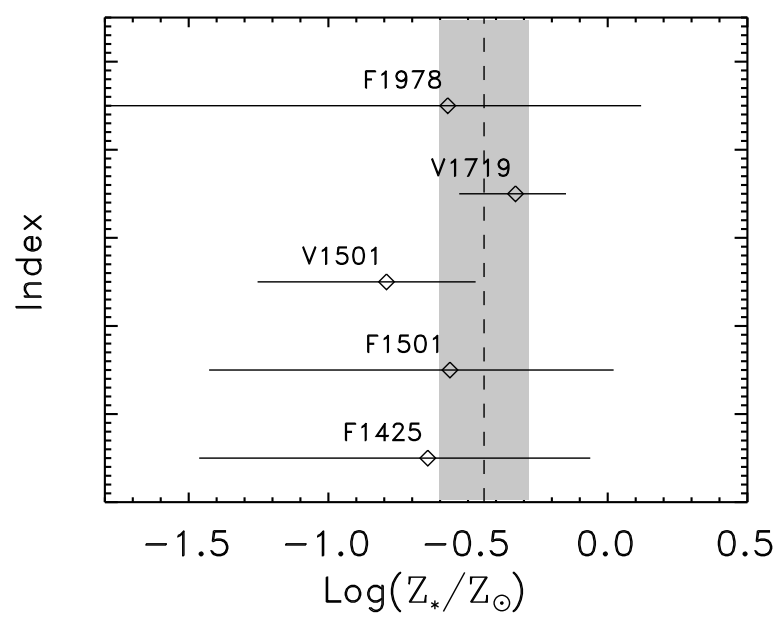

Fig. 13. Stellar metallicity values derived from the co-added UV spectrum of 26 SFG members using the UV indices described in Sect. 5.3.3, and listed in Table 4 (black diamonds). The vertical dashed line represents the error-weighted mean, and the gray area the associated $1 \sigma$ uncertainty.

\subsubsection{Comparison with SFG UV spectra}

To investigate whether the observed UV spectral properties are peculiar to our protocluster members or consistent with those observed in field galaxies, we compare our stacked spectrum with those obtained in other SFGs from the literature. This comparison is useful to identify similarities with specific types of galaxies that can potentially help us to interpret our spectrum in a model-independent way, and might also pinpoint possible artifacts in our stack due to the low number of stacked sources and to the spectral noise.

High signal-to-noise UV spectra for SFGs at $z \gtrsim 2$ are available from stacking Lyman Break Galaxies (LBG; Shapley et al. 2003), and Ly $\alpha$ emitters (LAE; Cullen et al. 2019, 2020). We collected 12 composite spectra for LAEs at $z \simeq 3.5$ divided in bins of stellar mass, or Ly $\alpha \mathrm{EW}$, and one for LBGs at $z \simeq 3$. Half of these spectra have continuum slopes inconsistent with ours, among the remaining half only two composite spectra exhibit both continuum slope and spectral features consistent with our stacked spectrum. The two most similar composite spectra are obtained from the VANDELS-m3 LAE sample (i.e., 153 galaxies with $\log \left(\mathcal{M} / M_{\odot}\right)=9.20-9.50$, and $2.30 \leq z \leq 5.0$; Cullen et al. 2019), and the VANDELS-Q3 sample (LAEs with $\log \left(\mathcal{M} / M_{\odot}\right)=9.54 \pm 0.44$, and $3.0 \leq z \leq 5.0$; Cullen et al. 2020). The $\mathrm{m} 3$ and Q3 composite spectra are compared with our stack in Fig. 14. The two samples of LAEs with UV spectra similar to our stack, m3 and Q3, are best fitted with stellar models with metallicity $Z_{*}=0.0017 \pm 0.0001$, and $0.0021 \pm 0.0002$, respectively (assuming $Z_{\odot}=0.0142$; Asplund et al. 2009). These are, respectively, about 0.5 , and 0.4 dex lower than the average metallicity of our protocluster members. This discrepancy is consistent with the difference in stellar mass, and redshift between our sample and the $\mathrm{m} 3$ and Q3 samples. Indeed, the mean stellar mass of the 26 SFGs used in the stacked spectrum is $\log \left(\mathcal{M} / M_{\odot}\right)=10.0 \pm 0.44$ (see Sect. 5.4), about 0.5 dex larger than the LAE median stellar mass. There is a well know relation between stellar mass and metallicity in SFGs that evolves with redshift (see e.g., Calabrò et al. 2021) that reconciles the value obtained for our protocluster members with those derived for these LAE samples.

On the other hand LAEs are, typically, more metal poor than SFGs at the same stellar mass and redshift (Finkelstein et al.
2011), suggesting that our members might be more metal poor than typical SFGs at the same redshift. Similar results have been obtained in other protoclusters at $z \sim 2$ (Chartab et al. 2021; Sattari et al. 2021), and interpreted as a sign of pristine cold gas accretion, but this is not observed in all protoclusters (see e.g., Shimakawa et al. 2015).

\subsection{Members stellar masses and SFRs}

To determine the activity level and evolutionary phase of the selected protocluster members, we estimate their stellar mass and SFR and compare them with the scaling relations that describe normal SFGs, starburst galaxies, and quiescent galaxies.

To estimate stellar masses and SFRs of the spectroscopic members, we fit their SEDs with galaxy models. The SEDs are obtained from the L16 Cosmos multiwavelength catalog. We use $3^{\prime \prime}$ diameter aperture-corrected magnitudes, and apply the relative photometric and systematic offsets following L16 Eq. (9). Magnitudes are also corrected for foreground galactic extinction using the given reddening $E(B-V)$ values and extinction factors following L16 Eq. (10). We use data in 20 broad-band filters, including CFHT/MegaPrime $u$, CFHT/WIRCam $H$, and $K_{s}$, SUBARU/SuprimeCamera $B, V, r, i, z^{++}$, VISTA/VIRCAM $Y, J, H, K_{\mathrm{s}}$ from the ultra-VISTA 3 rd data release (McCracken et al. 2012), Spitzer/IRAC [3.6, 4.5, 5.8, 8.0 $\mu \mathrm{m}]$ and MIPS [24, 70 , and $160 \mu \mathrm{m}]$, and Herschel/SPIRE [250, 350, and $500 \mu \mathrm{m}$ ] bands. A flux estimate in the SPIRE band from either HerMES or L16 is available for ten of the 31 spectroscopic members. Those listed in L16 are all detections at $>3 \sigma$ and have been obtained using as prior the MIPS[24 $\mu \mathrm{m}]$ position. These are available for four sources at $250 \mu \mathrm{m}$, and for two at 350, and $500 \mu \mathrm{m}$ (see Table 3). In the remaining cases, we use the HerMES data and treat them as upper limits even if the flux measurement is $>3 \sigma$ because the selected source might not be the only counterpart to the SPIRE flux. Upper limits to the SPIRE fluxes are equivalent to $5 \sigma$, where $\sigma$ includes instrumental and confusion noise of the Cosmos HerMES observations (see Sect. 2; Oliver et al. 2012).

We use the Code Investigating GALaxy Emission (CIGALE; Noll et al. 2009; Ciesla et al. 2015; Boquien et al. 2019), with redshift equal to the spectroscopic one, to fit the observed SEDs. The model assumptions are: an exponentially declining star formation history with a random burst of star formation, the Bruzual \& Charlot (2003) stellar synthesis population models, a Chabrier (2003) IMF, solar metallicity, continuum and line nebular emission, the Calzetti et al. (2000) dust attenuation prescription, the Draine \& Li (2007) dust emission models (module dl2014), and an AGN component as modeled by Fritz et al. (2006). Ciesla et al. (2015) show that not taking an AGN component into account when fitting broad-band SEDs of AGN host galaxies might result in a bias in the estimated stellar mass and SFR. Since some of the selected sources might host an AGN, even if not detected in the spectrum or in the X-ray, we perform fits both with and without an AGN component for all sources. Based on the resulting $\chi^{2}$, the addition of an AGN component yields a better fit only in two cases, IDs 55326, and 58057. The SEDs of the other two AGN of the sample, IDs SL01 and L710971, do not produce better fits with an additional AGN component.

In Fig. E.1, we show the best fit SEDs and in Table 5 the stellar masses and SFRs obtained from the best fits.

The estimated stellar masses range from $\sim 0.2-20 \times 10^{10} M_{\odot}$. Compared to the characteristic mass $\mathcal{M}^{*}$ obtained by fitting 


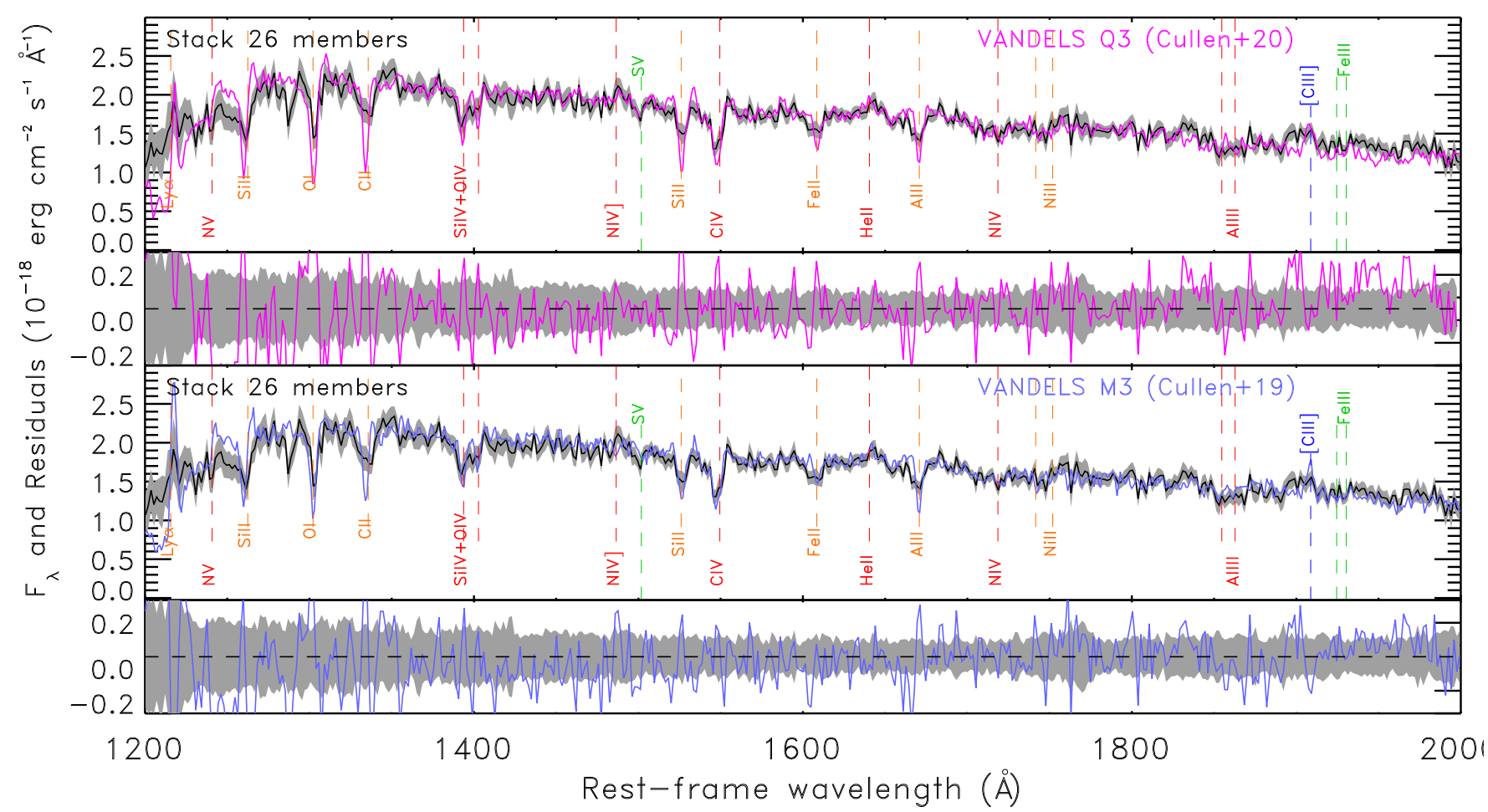

Fig. 14. Stacked spectrum (black solid line) and $1 \sigma$ uncertainty (gray filled area) compared with composite spectra obtained from stacking the VANDELS spectra of two LAE subsamples: Q3 (top panel: magenta line); and m3 (bottom panel: blue line) (Cullen et al. 2019, 2020). Below each panel residuals are plotted and the $1 \sigma$ uncertainty (gray shaded area) associated with our stack. The main spectral features are highlighted with vertical dashed lines and annotated.

the Schechter mass function of SFGs at $2.0<z<2.5$ (i.e., $\mathcal{M}^{*}=4_{-1.0}^{+1.6} \times 10^{10} M_{\odot}$; Davidzon et al. 2017), most $(81 \%)$ of the spectroscopic members are less massive, and only $13 \%$ (4 galaxies) are more massive than $\mathcal{M}^{*}$, with stellar masses $\simeq(1-$ 2) $\times 10^{11} M_{\odot}$. The four galaxies with $\mathcal{M}>10^{11} M_{\odot}$ include one AGN, and two SPIRE detected sources. The relatively low stellar masses for the other galaxies suggests that these have not yet reached their maximal mass, and might be at an early stage of evolution, as indicated by their UV spectral properties.

We examine whether there is a relation between the stellar mass and the location of a galaxy within the structure as previously found for the HAEs in the structure by Koyama et al. (2021). We find that the mean stellar mass in the core (i.e., $\left.\left\langle\log \left(\mathcal{M}^{\text {core }} / M_{\odot}\right)\right\rangle=10.7 \pm 0.4\right)$ is 0.5 dex larger than the average of all ss 1 members (i.e., $\left.\left\langle\log \left(\mathcal{M}^{\text {ssl } 1} / M_{\odot}\right)\right\rangle=10.2 \pm 0.4\right)$, but this difference is not highly significant considering the wide range of stellar masses. We plot the spatial distribution of the members of ss1 in Fig. 15. Two of the most massive galaxies (IDs: SL01, and SL03) are in the core, while the four least massive ones are in the most external regions. We note that the three galaxies in the core are also HAEs. The CIGALE mass estimates are consistent with those estimated in Koyama et al. (2021) for IDs SL01, and SL03, and $60 \%$ lower for ID 58057. Such a difference is probably due to the AGN component included in our fit, and not considered in Koyama et al. (2021). This analysis agrees with the results reported by Koyama et al. (2021), the most massive members are located in the structure core, although the enhancement of stellar mass in the core is less pronounced than found with the HAEs.

In terms of SFRs, they range from 5 to $\sim 250 M_{\odot} \mathrm{yr}^{-1}$, with mean $\left\langle\mathrm{SFR}^{\mathrm{ss} 1}\right\rangle=33 \pm 25\left(M_{\odot} \mathrm{yr}^{-1}\right)$ for all ss 1 members, and $\left\langle\mathrm{SFR}^{\text {core }}\right\rangle=73 \pm 52 M_{\odot} \mathrm{yr}^{-1}$ in the core. There is thus also a higher average SFR in the core than in the whole ss1 structure.
This is also illustrated in the middle panel of Fig. 15, where we show the spatial distribution of the sources in ss 1 as a function of their SFR. The figure shows the core having more members with high SFRs than the outer regions, but as in the case of stellar mass the difference is not highly significant. The estimated total SFR for the six HAEs in our spectroscopic sample is $544 M_{\odot} \mathrm{yr}^{-1}$ from the dust-corrected $\mathrm{H} \alpha$ luminosity, and $440 \pm 43 M_{\odot} \mathrm{yr}^{-1}$ from our SED fitting. The six objects in common include two AGN, but, interestingly, the SFRs estimated for the two AGN are consistent, while the $\mathrm{H} \alpha$-derived SFRs are systematically larger than our estimates for the non-AGN HAEs that are not detected by SPIRE in the sub-mm. This comparison suggests that our CIGALE-based SFR estimates might be underestimated by $20 \%$ on average. The sources with the highest SFRs (i.e., SFR $>100 M_{\odot} \mathrm{yr}^{-1}$ ) are the four detected by SPIRE, IDs 55326, 60984, SL03, and 58057.

To establish whether the estimated SFRs are consistent with normal SFGs, or starbursting galaxies, we compute the offset from the main sequence (MS) relation at $z=2.17$ (Speagle et al. 2014; Elbaz et al. 2011; Rodighiero et al. 2011). Such an offset is the ratio between the estimated SFR and the expected SFR based on the object stellar mass and assuming the MS relation at the source redshift. The SFR as a function of stellar mass for all 31 spectroscopic members is shown in Fig. 16. The dotted lines represent the scatter around the MS, which is about a factor of 3 . We find that $94 \%$ of the spectroscopic members are on the MS, with only two sources below, in the region where quenching galaxies are expected. None of the selected members is in the starburst region. No clear correlation is observed between the MS-offset and a source location within the structure. One AGN, ID SL01, is below the MS and it is also the closest source to the quiescent wedge in the UVJ and NUVrK diagrams. This source might be going through a quenching phase. We note that 
Table 5. CIGALE best-fit parameters of the spectroscopic members in the G237 field.

\begin{tabular}{|c|c|c|c|}
\hline ID & $\begin{array}{c}\mathcal{M} \\
\left(10^{10} M_{\odot}\right)\end{array}$ & $\begin{array}{c}\mathrm{SFR} \\
\left(M_{\odot} \mathrm{yr}^{-1}\right)\end{array}$ & $\log \left(\mathrm{SFR} / \mathrm{SFR}_{\mathrm{MS}}\right)^{(a)}$ \\
\hline \multicolumn{4}{|c|}{$\mathrm{ss} 1: z=2.15-2.164$} \\
\hline $55326^{(b)}$ & $1.64 \pm 0.46$ & $119 \pm 10$ & 0.46 \\
\hline 55687 & $17.01 \pm 0.85$ & $5.3 \pm 1.8$ & -1.66 \\
\hline 56014 & $0.28 \pm 0.06$ & $14 \pm 2$ & 0.14 \\
\hline 56915 & $1.26 \pm 0.17$ & $30 \pm 4$ & -0.05 \\
\hline 57557 & $0.80 \pm 0.14$ & $43 \pm 5$ & 0.26 \\
\hline 58020 & $2.12 \pm 0.26$ & $44 \pm 4$ & -0.05 \\
\hline $58057^{(b)}$ & $5.08 \pm 1.00$ & $143 \pm 26$ & 0.17 \\
\hline 58173 & $0.61 \pm 0.08$ & $14 \pm 2$ & -0.14 \\
\hline 58200 & $0.54 \pm 0.18$ & $9.4 \pm 8.3$ & -0.27 \\
\hline 58206 & $1.79 \pm 0.51$ & $51 \pm 15$ & 0.06 \\
\hline 58266 & $1.22 \pm 0.13$ & $10.7 \pm 6.1$ & -0.48 \\
\hline 59504 & $0.36 \pm 0.04$ & $14.7 \pm 1.3$ & 0.06 \\
\hline 60718 & $0.75 \pm 0.11$ & $10.4 \pm 2.4$ & -0.33 \\
\hline 60811 & $2.03 \pm 0.29$ & $91 \pm 14$ & 0.28 \\
\hline L710971 & $3.78 \pm 1.10$ & $52 \pm 30$ & -0.18 \\
\hline SL01 & $12.60 \pm 1.67$ & $34 \pm 23$ & -0.76 \\
\hline SL03 & $18.67 \pm 1.09$ & $158 \pm 19$ & -0.22 \\
\hline 54698 & $0.49 \pm 0.08$ & $26 \pm 3$ & 0.20 \\
\hline 57138 & $0.94 \pm 0.15$ & $76 \pm 8$ & 0.45 \\
\hline 57730 & $0.73 \pm 0.21$ & $57 \pm 12$ & 0.41 \\
\hline \multicolumn{4}{|c|}{$2.164<z<2.19$} \\
\hline 54765 & $0.53 \pm 0.08$ & $9 \pm 1$ & -0.31 \\
\hline 55951 & $1.30 \pm 0.17$ & $18 \pm 3$ & -0.29 \\
\hline 56005 & $0.60 \pm 0.10$ & $29 \pm 5$ & 0.18 \\
\hline \multicolumn{4}{|c|}{$\operatorname{ss} 2: z=2.19-2.20$} \\
\hline 54677 & $2.65 \pm 0.56$ & $37 \pm 24$ & -0.21 \\
\hline 56950 & $0.87 \pm 0.35$ & $35 \pm 19$ & 0.13 \\
\hline 57695 & $0.64 \pm 0.19$ & $47 \pm 12$ & 0.37 \\
\hline 59496 & $0.57 \pm 0.12$ & $18 \pm 3$ & -0.02 \\
\hline 59906 & $0.64 \pm 0.09$ & $23 \pm 2$ & 0.05 \\
\hline 60026 & $0.21 \pm 0.04$ & $8 \pm 1$ & -0.03 \\
\hline 60355 & $0.91 \pm 0.12$ & $15 \pm 2$ & -0.26 \\
\hline 60984 & $14.47 \pm 1.45$ & $247 \pm 24$ & 0.05 \\
\hline \multicolumn{4}{|c|}{ full: $2.15<z<2.20$} \\
\hline Total & $96 \pm 3$ & $1485 \pm 71$ & 0.22 \\
\hline
\end{tabular}

Notes. ${ }^{(a)}$ Logarithm of the ratio between the estimated SFR and the expected SFR assuming the main sequence relation (Speagle et al 2014) at the source redshift and stellar mass corrected for a Chabrier (2003) IMF. ${ }^{(b)}$ The AGN fraction, or contribution to the bolometric luminosity is $5 \pm 2 \%$ in 55326, and $6 \pm 4 \%$ in 58057 .

since this source is very faint in the $\mathrm{ACS} / F 814 W$-band image (see Sect. 5.6), it is not possible to investigate whether a morphological transformation is occurring, leaving a bulge-dominated galaxy.

\subsection{AGN in the structure}

Determining the density and power of AGN in high-redshift structures is of great interest as feedback from AGN is often invoked as one of the main mechanisms to halt star-formation in their host galaxies, and to ionize and enrich the intergalactic medium. To identify AGN activity in the G237 spectroscopic members, we consider the spectra and the X-ray data available in the Cosmos field (the Chandra and XMM-Newton X-ray cat-
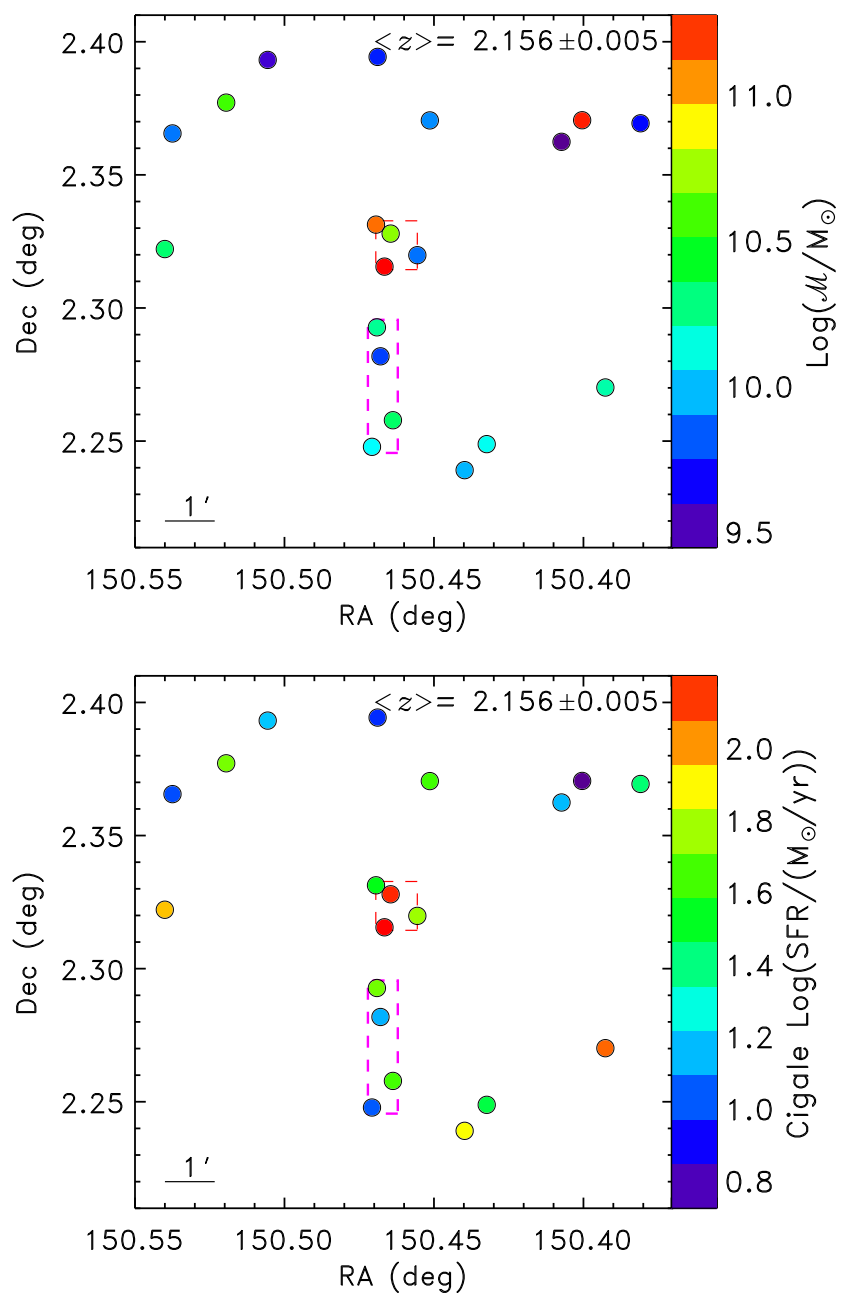

Fig. 15. Spatial distribution of all spectroscopic sources with $z=2.15-$ 2.16 with colors corresponding to the estimated stellar mass (top panel), and SFR (bottom panel). Dashed red and magenta rectangles delineate the structure components A, B described in Sect. 3.4, and shown in Fig. 6.

alogs; Cappelluti et al. 2009; Civano et al. 2016). We could also consider the AGN fraction estimated from the CIGALE SEDfitting, but the scarcity of MIR detections, makes this parameter too unreliable to securely identify AGN. Nonetheless, CIGALE identifies AGN in only two sources, both already known AGN based on other diagnostic criteria. In ss 1, three sources (IDs 55326, 58057, and SL01) exhibit broad emission lines (i.e., $\mathrm{H} \alpha$, Ly $\alpha$, C IV 21549) in their spectra, implying the presence of a broad line (type 1 or unobscured) AGN. Two of these three AGN are also X-ray detected. There is a third source that is $\mathrm{X}$-ray detected, ID L710971, but with no strong C IV $\lambda 1549$ emission, implying that it is an obscured (type 2) AGN. We thus find, in total, four AGN out of 20 members $(20 \pm 10 \%)$, of which three $(15 \pm 9 \%)$ are X-ray sources. The total $(0.5-10 \mathrm{keV})$ (not corrected for absorption) X-ray luminosities of the three $X$-ray detected sources are all $>4 \times 10^{43} \mathrm{erg} \mathrm{cm}^{-2} \mathrm{~s}^{-1}$, implying that they are powered by an AGN (Alexander et al. 2005). The fraction of X-ray detected AGN (15\%) is consistent with those measured in other two protoclusters at $z<2.25\left(17_{-6.0}^{+8.6 \%}\right.$ in $\mathrm{Cl} 00218.3-0510$ at $z=1.6$, and $17_{-9.1}^{+16.2 \%}$ in 2QZ 1004+00 at $z=2.23$; Krishnan et al. 2017; Lehmer et al. 2013), and higher than in protoclusters at higher redshift $\left(4_{-2}^{+8} \%\right.$ in $\mathrm{HS} 1700+643$ at $z=2.31,2 \pm 1.3 \%$ in USS $1558-003$ at $z=2.53$, and $5.1 \pm 3.3 \%$ in 


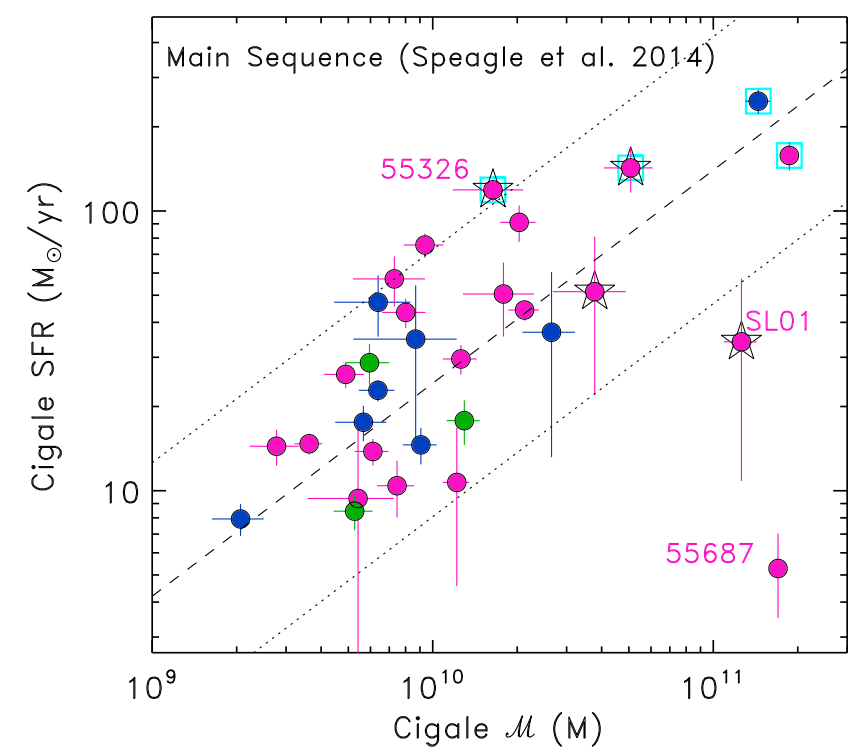

Fig. 16. SFR as a function of stellar mass for the 31 spectroscopic members (full circles, magenta: $2.15 \leq z \leq 2.164$, green: $2.164<z<2.19$, and blue: $2.19 \leq z \leq 2.20$ ). AGN are indicated with a black star, and sources detected by SPIRE with a cyan square. The black dashed line is the MS relation at $z=2.17$ as formulated by Speagle et al. (2014) (corrected for a Chabrier (2003) IMF), and the black dotted lines represent a factor of 3 scatter around the MS.

SSA22 at $z=3.09$; Digby-North et al. 2010; Macuga et al. 2019; Lehmer et al. 2009). When considering the 11 sources at $2.164<$ $z<2.20$, no X-ray detected source nor broad line AGN is found. Thus, including these additional sources, the AGN fraction in the entire structure would be $13 \pm 6 \%$, still consistent with an excess of AGN activity with respect to the field. Two of the four SPIRE-detected members, IDs, 58057 and 55326, are Xray luminous sources $\left(L_{\mathrm{X}}>7 \times 10^{43} \mathrm{erg} \mathrm{s}^{-1}\right)$, corresponding to $50 \pm 35 \%$. This fraction is much higher than the fraction of $X-$ ray detected SMGs in the field (i.e., 11-29\% SMGs are X-ray detected in the field with any X-ray luminosity; Johnson et al 2013; Georgantopoulos et al. 2011; Laird et al. 2010). The simultaneous sub-mm and X-ray emission in these objects, although based on a few sources, is intriguing and will be discussed later.

In the $10^{\prime} \times 12^{\prime}$ region centered on $\mathrm{G} 237$, there are $74 \mathrm{X}$-ray sources, or $0.62 \pm 0.07 \mathrm{arcmin}^{-2}$. Out of these $74 \mathrm{X}$-ray sources, 45 have a spectroscopic redshifts and three $(4 \%)$ of them are structure members. For the remaining 29 X-ray sources, a photometric redshift is available for 24 sources and none of them falls in the structure redshift range 2.15-2.20. We can thus say that there are at least three X-ray detected members in the Cosmos released X-ray catalogs (Cappelluti et al. 2009; Civano et al. 2016), and eight at the most, if we include the five sources for which no redshift estimate is available.

The three confirmed members have X-ray $(0.5-10 \mathrm{keV})$ luminosities greater than $4.4 \times 10^{43} \mathrm{erg} \mathrm{cm}^{-2} \mathrm{~s}^{-1}$, and they all belong to ss1. Assuming a volume density of $(0.2-$ 1) $\times 10^{-4} \mathrm{Mpc}^{-3}$ for X-ray sources with similarly high luminosities at $z \sim 2-3$ (Silverman et al. 2008; Fotopoulou et al. 2016; Ranalli et al. 2016), the expected number of X-ray sources in the volume of $\mathrm{ss} 1\left(4376 \mathrm{Mpc}^{3}\right)$ is $\leq 0.44$ sources. We thus find a number of luminous $\mathrm{X}$-ray sources in the structure that is $\sim 7$ times larger than expected, which corresponds to an X-ray source overdensity of $\delta_{\mathrm{X} \text {-ray }}=(3-0.44) / 0.44=5.8$.

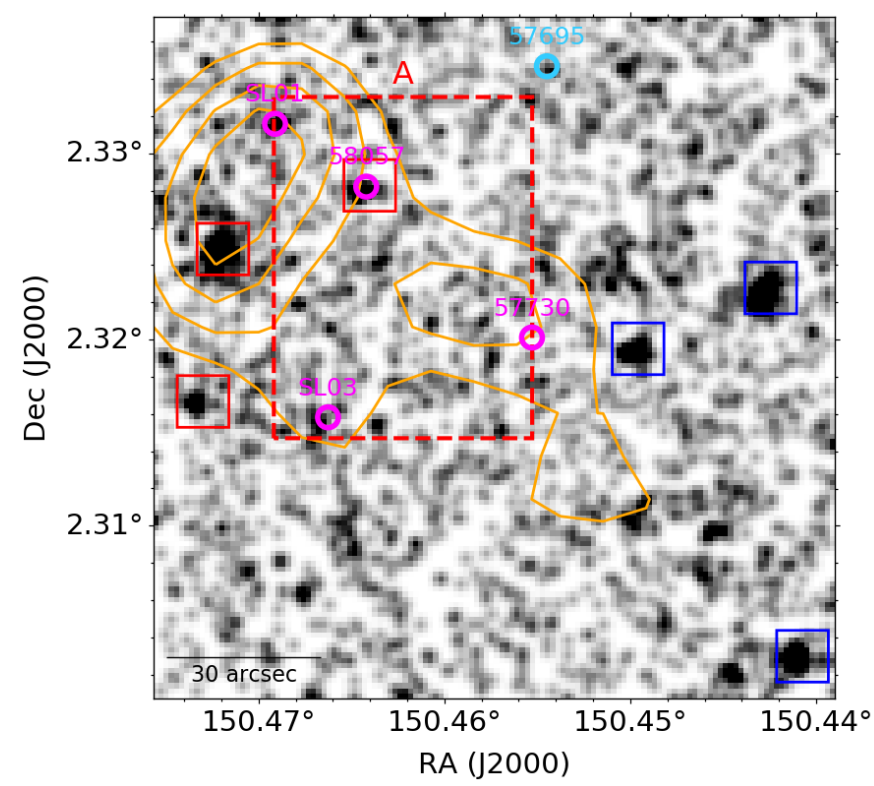

Fig. 17. Chandra $0.5-7 \mathrm{KeV}$ rebinned $2.2^{\prime} \times 2.2^{\prime}$ image of the protocluster core. Spectroscopic members are shown with $3^{\prime \prime}$ radius circles (magenta if $2.15 \leq z<2.164$, light blue if $2.19<z<2.20$ ), and X-ray detected sources with $10^{\prime \prime} \times 10^{\prime \prime}$ boxes (red if detected by both Chan$d r a$ and XMM-Newton, and blue if detected only by Chandra). There are several X-ray sources missing in the released Chandra Cosmos catalog, including IDs SL01, and probably SL03, and 57695. SPIRE $350 \mu \mathrm{m}$ contours are shown in orange. The red rectangle shows the boundaries of the protocluster core (component A in Sect. 3.4).

In a $2.2^{\prime} \times 2.2^{\prime}$ region centered on the protocluster core there are six X-ray sources. In Fig. 17, we show the Chandra $0.5-7 \mathrm{keV}$ image of such a region and superimpose all the detected X-ray sources from the Cosmos released Chandra and XMM-Newton X-ray catalogs (Cappelluti et al. 2009; Civano et al. 2016), and the structure spectroscopic members. Out of the six X-ray sources, only one (14\%), ID 58057, belongs to the structure, the others five are at $z<2$ (based on two spectroscopic, and three photometric redshifts). The Chandra image shown in Fig. 17 shows, however, the presence of other X-ray sources that are not present in the public Chandra catalog, including some spectroscopic members, ID SL01, and possibly IDs SL03, and 57695. No other spectroscopic sources with visible X-ray emission in the Chandra image, and missing in the released X-ray catalog, have been found in the rest of the field. SL01, and SL03 belong to ss1, and 57695 to ss2. ID SL01 was already taken into account in the AGN fraction estimate as it is spectroscopically classified as an AGN, if we also include ID SL03, the AGN fraction in ss 1 increases to $25 \pm 11 \%$. There is thus strong evidence of an excess of X-ray sources in the protocluster core. Including the two spectroscopically classified AGN also located in the core, we can state that this region shows an enhanced fraction of AGN. The mechanisms that favor the presence of AGN might be also responsible for the enhanced level of SF activity.

\subsection{Members morphology}

A morphological analysis of the galaxy members within assembling structures can reveal whether, when and where galaxies experience morphological transformation and which processes are the main drivers (e.g., mergers, close encounters, gas stripping, etc.). A morphological classification in early-type (ET), 
Table 6. $\mathrm{PHz}$ G237.01+42.50 spectroscopic members.

\begin{tabular}{|c|c|c|c|c|c|c|c|c|c|}
\hline ID & $\begin{array}{r}\alpha_{J 2000} \\
(\mathrm{deg})\end{array}$ & $\begin{array}{l}\delta_{J 2000} \\
(\mathrm{deg})\end{array}$ & Number & $z_{\text {spec }}$ & $z_{f l g}^{(a)}$ & Type ${ }^{(b)}$ & $\begin{array}{c}\operatorname{mag}(\mathrm{Ks}) \\
(\mathrm{AB})\end{array}$ & $z_{\text {phot }}$ & Comp./Note ${ }^{(c)}$ \\
\hline \multicolumn{10}{|c|}{ Substructure 1: $z=2.15-2.164$} \\
\hline 55326 & 150.39265 & 2.270105 & 639096 & 2.1576 & 14 & ET & $22.189 \pm 0.022$ & 1.8205 & CXO-ID: 769 \\
\hline 55687 & 150.40045 & 2.370529 & 707504 & 2.1529 & 1 & Disk & $20.455 \pm 0.006$ & 1.4264 & \\
\hline 56014 & 150.40732 & 2.362427 & 701268 & 2.1580 & 1 & Irr & $23.221 \pm 0.049$ & 2.3446 & \\
\hline 56915 & 150.43237 & 2.248860 & 624965 & 2.1576 & 2 & Disk & $22.483 \pm 0.020$ & 2.1418 & \\
\hline 57557 & 150.45144 & 2.370448 & 706805 & 2.1520 & 1 & Disk & $22.779 \pm 0.023$ & 1.6211 & \\
\hline 58020 & 150.46384 & 2.257829 & 631032 & 2.1519 & 3 & $\operatorname{Irr}^{(d)}$ & $21.902 \pm 0.011$ & 2.1821 & B/HAE \\
\hline 58057 & 150.46451 & 2.327942 & 677305 & 2.1517 & 13 & Disk & $21.612 \pm 0.008$ & 2.0626 & A/CXO-ID: 1265 , HAE \\
\hline 58173 & 150.46791 & 2.281895 & 646606 & 2.1520 & 2 & Irr & $22.984 \pm 0.029$ & 1.654 & B/non HAE \\
\hline 58200 & 150.46892 & 2.394305 & 721845 & 2.1520 & 2 & $\ldots$ & $23.410 \pm 0.042$ & 1.7865 & \\
\hline 58206 & 150.46911 & 2.292743 & 653383 & 2.1527 & 3 & $\operatorname{Disk}^{(d),(e)}$ & $22.675 \pm 0.015$ & 1.99 & $\mathrm{~B} / \mathrm{HAE}$ \\
\hline 58266 & 150.47072 & 2.247844 & 625320 & 2.1502 & 2 & $\operatorname{Irr}^{(d)}$ & $22.489 \pm 0.037$ & 2.1169 & B/HAE \\
\hline 59504 & 150.50562 & 2.393203 & 721608 & 2.1500 & 2 & Disk & $22.715 \pm 0.027$ & 2.2462 & \\
\hline 60718 & 150.53749 & 2.365551 & 703833 & 2.1510 & 2 & $\operatorname{Disk}^{(e)}$ & $22.638 \pm 0.030$ & 1.5001 & \\
\hline 60811 & 150.54004 & 2.322157 & 673721 & 2.1547 & 1 & $\operatorname{Disk}^{(d)}$ & $21.729 \pm 0.012$ & 1.4376 & \\
\hline L710971 & 150.51958 & 2.377024 & 710971 & 2.1600 & 1 & $\ldots$ & $22.645 \pm 0.025$ & 2.6792 & CXO-ID: 1746 \\
\hline SL01 & 150.46945 & 2.331292 & 679779 & 2.1586 & 14 & $\ldots$ & $21.379 \pm 0.008$ & 2.1975 & $\mathrm{~A} / \mathrm{HAE}$ \\
\hline SL03 & 150.46652 & 2.315597 & 669706 & 2.1592 & 3 & Disk & $20.584 \pm 0.005$ & 2.2488 & $\mathrm{~A} / \mathrm{HAE}$ \\
\hline 57138 & 150.43973 & 2.239109 & 618296 & 2.1615 & 4 & Disk & $22.198 \pm 0.013$ & 2.1485 & \\
\hline 57730 & 150.45557 & 2.319829 & 672385 & 2.1637 & 1 & Disk & $22.566 \pm 0.020$ & 1.4109 & A/non HAE \\
\hline 54698 & 150.38090 & 2.369391 & 706004 & 2.1637 & 2 & $\operatorname{Irr}(d)$ & $22.667 \pm 0.053$ & 0.8765 & \\
\hline \multicolumn{10}{|c|}{$2.164<z<2.19$} \\
\hline 54765 & 150.38211 & 2.323130 & 673531 & 2.1757 & 1 & Disk & $23.731 \pm 0.123$ & 1.9198 & \\
\hline 55951 & 150.40584 & 2.366063 & 703555 & 2.1815 & 1 & Disk & $22.441 \pm 0.020$ & 2.1324 & \\
\hline 56005 & 150.40720 & 2.255304 & 629074 & 2.1700 & 2 & Disk & $22.899 \pm 0.029$ & 2.1825 & \\
\hline \multicolumn{10}{|c|}{ Substructure $2: z=2.19-2.20$} \\
\hline 54677 & 150.38052 & 2.274505 & 641998 & 2.1986 & 4 & Irr & $21.728 \pm 0.022$ & 2.2415 & \\
\hline 56950 & 150.43380 & 2.222876 & 607540 & 2.1991 & 1 & Irr & $22.641 \pm 0.021$ & 1.4602 & \\
\hline 57695 & 150.45481 & 2.334413 & 682054 & 2.1915 & 1 & Irr & $22.506 \pm 0.020$ & 2.1538 & non HAE \\
\hline 59496 & 150.50540 & 2.237819 & 617293 & 2.1909 & 4 & Disk & $22.770 \pm 0.021$ & 2.0405 & C/non HAE \\
\hline 59906 & 150.51611 & 2.398838 & 725166 & 2.1907 & 2 & $\operatorname{Irr}^{(d)}$ & $22.555 \pm 0.024$ & 2.1756 & \\
\hline 60026 & 150.51934 & 2.252120 & 626986 & 2.1916 & 2 & Disk & $23.665 \pm 0.046$ & 2.216 & C/non HAE \\
\hline 60355 & 150.52715 & 2.270970 & 639939 & 2.1993 & 4 & Irr & $22.503 \pm 0.017$ & 2.2373 & $\mathrm{C}$ \\
\hline 60984 & 150.54539 & 2.257720 & 631549 & 2.1982 & 1 & Irr & $20.738 \pm 0.004$ & 1.7994 & $\mathrm{C}$ \\
\hline
\end{tabular}

Notes. ${ }^{(a)}$ zflg is a flag that indicates the reliability of the redshift estimate. Based on the zCosmos-Deep sample (Lilly et al. 2007), the estimated redshifts are expected to be correct in $50 \%$ of the cases for $z f g=1$, in $85 \%$ for $z f g=2$, and in $100 \%$ for $z f g=3$, or 4 . In case of AGN spectral features in the spectrum, $z f g$ is incremented by $10 .{ }^{(b)}$ Morphological type based on the ACS I-band image (Disk, ET: early-type, Irr: irregular) from the Cosmos morphological catalogs. A morphological type is not available when a source is too faint. ${ }^{(c)}$ The letter A, B, or C indicates the component described in Sect. 3.4. Chandra IDs are listed in case of X-ray detection. Sources within the Subaru/MOIRCS FOV are listed as HAE if selected as such, or as non-HAE otherwise (see Sect. 3.3, and Koyama et al. 2021). ${ }^{(d)}$ Presence of a nearby galaxy at $<1.8^{\prime \prime}, \sim 15 \mathrm{kpc}$ in the ACS I-band image. ${ }^{(e)}$ Morphological classification derived from visual inspection of the ACS $I$-band images because not available in the Cosmos morphological catalogs.

disk, and irregular galaxy is available from the Cosmos morphological catalogs for 26 members ${ }^{11}$. This morphological classification is based on the HST/ACS $F 814 W$-band images (corresponding to $\sim 2500 \AA$ in the rest-frame), and on the asymmetry, concentration, clumpiness parameters, and the Gini index as described in Cassata et al. (2007), Tasca et al. (2009). For five sources a morphological classification is not available. Two of them exhibit an elongated structure in the HST/ACS $F 814 W$-band image, and can thus be classified as disks. The remaining three sources are extremely faint and extended to be morphologically classified through a visual inspection. The morphological classification of all 31 members is listed in Table 6 .

\footnotetext{
${ }^{11}$ https://irsa.ipac.caltech.edu/data/CoSMos/overview. html
}

Postage stamps $\left(6^{\prime \prime} \times 6^{\prime \prime}\right)$ in the HST/ACS $F 814 W$-band and in the UltraVISTA/YJH bands of all member candidates are shown in Figs. D.1-D.3.

It is also interesting to investigate the presence of a nearby galaxy that might be indicative of an interactive system, like a merger. We searched for potential companion galaxies using the L16 catalog and by visually inspecting the postage stamps. We consider a pair of galaxies to be likely interacting if the projected distance is $\leq 15 \mathrm{pkpc}$ (equivalent to $\leq 1.8^{\prime \prime}$ at the structure redshift). We note that larger physical separations are observed in interacting pairs, but would be more consistent with a premerger phase (see e.g., Ventou et al. 2017). We find in total six sources with a potential companion, three from the L16 catalog (IDs 58206, 59906, and 54698), and three additional ones by the images visual inspection (IDs 58020, 58266, and 60811; see 
Table 7. Overdensity properties.

\begin{tabular}{|c|c|c|c|c|c|c|c|c|c|c|c|c|}
\hline Structure & $z$ range & $\begin{array}{c}N \\
\mathrm{G} 237\end{array}$ & $\begin{array}{c}\langle N\rangle \\
\text { Field }\end{array}$ & $\delta_{\text {gal }}$ & $\sigma_{\delta_{\mathrm{gal}}}$ & Extent & $\begin{array}{c}V_{\mathrm{app}} \\
\left(\mathrm{cMpc}^{3}\right)\end{array}$ & $C$ & $\delta_{\mathrm{m}}$ & $\delta_{\mathrm{L}}\left(z_{\mathrm{obs}}\right)$ & $\delta_{\mathrm{L}}(z=0)$ & $\begin{array}{l}M_{\mathrm{h}}(z=0) \\
\left(10^{14} M_{\odot}\right)\end{array}$ \\
\hline Full & & 29 & $10.5 \pm$ & 1.8 & 5. & $9.9^{\prime} \times 10.6^{\prime}$ & 18532 & 0.80 & 0.72 & 0.46 & 1.15 & $15.7_{-32}^{+6.0}$ \\
\hline ss 1 & 164 & 18 & $3.3 \pm 2.7$ & 4.5 & 5 & $9.5^{\prime} \times 9.3^{\prime}$ & 4376 & 0.66 & 1.4 & 0.70 & 1.75 & $6.6^{+26.0}$ \\
\hline ss2 & $2.19-2.20$ & 8 & $1.6 \pm 1.3$ & 3.9 & 5.0 & $9.9^{\prime} \times 10.6^{\prime}$ & 3724 & 0.68 & 1.35 & 0.67 & 1.69 & $5.1_{-19}^{+19.7}$ \\
\hline
\end{tabular}

Notes. $N$ is the number of sources with spectroscopic redshift in the Cosmos field from the catalogs listed in Sect. 3.2. We note that the sources from our LBT/LUCI program are not taken into account to estimate the galaxy overdensity. $\langle N\rangle$ is the average number of sources with spectroscopic redshift in the Cosmos field after excluding the G237 region. $\delta_{\mathrm{gal}}$, and $\sigma_{\delta_{\mathrm{gal}}}$ are, respectively, the density contrast and significance of the galaxies in $\mathrm{G} 237$ in the given redshift ranges. Extent is the area projected on the sky where the galaxies in the defined redshift ranges are distributed, and $V_{\text {app }}$ the volume obtained by multiplying the extent by the distance along the line of sight given by the redshift range. $C$ is the parameter that takes into account the redshift distortions (see text and Steidel et al. 1998). $\delta_{\mathrm{m}}$ is the matter overdensity obtained as described in Sect. 6.1, and used in Eq. (2) to derive the $z=0$ halo mass $M_{\mathrm{h}}(z=0)$ reported in the last column. $\delta_{\mathrm{L}}\left(z_{\mathrm{obs}}\right)$, and $\delta_{\mathrm{L}}(z=0)$ are the linear matter enhancements at the observed redshift and at $z=0$, respectively. An overdensity is expected to collapse when $\delta_{\mathrm{L}}$ reaches the collapse threshold $\delta_{\mathrm{c}}=1.686$ (Percival 2005). Thus only the two substructures, and not the full structure, are expected to collapse by $z=0$.

Figs. D.1-D.3, and Table 6). The photometric redshifts, available only for three companions, are too uncertain to confirm or rule out any interaction within each pair.

All members are either disks or irregular galaxies, as expected for a SFG. There is only one exception, ID 55326, classified as ET, but since it hosts an AGN, it is possible that the light profile is dominated by the AGN point-like emission. We do not see any difference in the morphological classification in the different substructures (e.g., in the core, or in the outer regions). About $40 \%$ of members are classified as irregular galaxies, and $19 \%$ have a close companion, suggesting that several members might be or have been experiencing an interaction. Three out of the four sources in the elongated structure (B component in Sect. 3.4) have a companion. Although the pair fraction in this component is much higher than in the rest of the structure, it would be difficult to draw any conclusion being based on a handful of sources and lacking spectroscopic confirmation for all pairs.

\section{Discussion}

\subsection{Is G237 a protocluster?}

A high- $z$ overdensity is considered a protocluster if it will collapse and relax by $z=0$, and its predicted halo mass at $z=0$, $M_{\mathrm{h}}(z=0)$, is $>10^{14} M_{\odot}$ (Chiang et al. 2013). In addition to being used to confirm the protocluster nature of a high- $z$ overdensity, $M_{\mathrm{h}}(z=0)$ is also useful to establish a connection between a protocluster and the analogous of its descendant, to explore the diversity across protoclusters, and the effect of the halo mass on the protocluster assembly and on the evolution of its members. There are various methods in the literature to estimate the $z=0$ halo mass (see e.g., Steidel et al. 1998; Cucciati et al. 2014; Long et al. 2020). Here, we adopt the widely used analytical method first applied by Steidel et al. (1998). This method provides an estimate of the total mass of the descendant cluster at $z=0$ from the (comoving) volume $V$ occupied by the overdensity, the mean density of the Universe $\bar{\rho}$ at the protocluster redshift, and the mass overdensity of the protocluster $\delta_{\mathrm{m}}$, through the following equation:

$M_{\mathrm{h}}=\bar{\rho} V\left(1+\delta_{\mathrm{m}}\right)$,

where $V$ is the true volume, $V \equiv V_{\text {apparent }} / C, \delta_{\mathrm{m}}$ is the mass overdensity that is related to the galaxy overdensity $\delta_{\text {gal }}$ through $1+b \delta_{\mathrm{m}}=C\left(1+\delta_{\mathrm{gal}}\right)$, where $b$ is the linear galaxy bias param- eter, and $\bar{\rho}=3.97 \times 10^{10} M_{\odot} \mathrm{Mpc}^{-3}$ for the cosmological parameters used in this work. The galaxy overdensities, $\delta_{\text {gal }}$, derived as described in Sect. 3.2 for the spectroscopic members in the full structure, and in the two substructures, and the corresponding comoving volumes are reported in Table 7 . The parameter $C$ takes into account the redshift distortions caused by peculiar velocities. Assuming that the structure is just breaking away from the Hubble flow, and following Steidel et al. (1998), $C$ can be approximated by $C=1+f-f\left(1+\delta_{\mathrm{m}}\right)^{1 / 3}$, where $f$ is the rate of growth of perturbations at the redshift of the protocluster (Lahav et al. 1991). In the cosmology adopted in this paper, $f=0.96$ at the mean redshift of the overdensity, $z=2.168$ (Lahav et al. 1991). We note that this method assumes that galaxies selected in a certain way predominantly occupy halos of the same mass, and this is expressed by the bias parameter. Here, we chose the bias parameter measured for galaxies at $z \simeq 2$ with stellar masses $\mathcal{M}>10^{9} M_{\odot}$ (i.e., $b=1.74$; Chiang et al. 2013). The $C$, and $\delta_{\mathrm{m}}$ values derived assuming these parameters for the different substructures, and the derived halo masses, $M_{\mathrm{h}}(z=0)$, are also listed in Table 7 . The uncertainty on $M_{\mathrm{h}}(z=0)$ is derived from the uncertainty on $\delta_{\text {gal }}$. This is derived assuming the galaxy field density $\pm 1 \sigma$, and by taking into account the redshift flag assigned to the spectroscopic redshifts in counting the galaxy members $^{12}$

The same estimate can be derived considering the overdensity of sub-mm galaxies $\left(\delta_{\mathrm{SMG}}=1.3\right)$ in ss1 and their bias parameter (i.e., $b=1.3$, Miller et al. 2015). In this case, we obtain $C=0.81$, and $\delta_{\mathrm{m}}=0.67$, and $M_{\mathrm{h}}(z=0)=3.6_{-1.3}^{+7.2} \times 10^{14} M_{\odot}$. This is consistent with the value derived from the spectroscopic members overdensity.

To establish whether these structure will collapse and virialize by $z=0$, we investigate the evolution of the structures overdensity in the linear regime of a spherical collapse model and compare the estimated linear matter enhancement at $z=0$, $\delta_{\mathrm{L}}(z=0)$ with the critical collapse threshold of $\delta_{\mathrm{c}}=1.686$ (Percival 2005). The $\delta_{\mathrm{L}}(z)$ values are derived from the $\delta_{\mathrm{m}}$ estimates using Eq. (18) in Mo \& White (1996). The $\delta_{\mathrm{L}}(z=0)$ values are obtained by multiplying $\delta_{\mathrm{L}}(z)$ by the linear growth factor $^{13}$ scaled by the cosmic scale factor (see Eq. (7) in

12 The number of spectroscopic members is weighted by the redshift flag as it follows, $n$ galaxies with $z f g=1$ count as $0.5 \times n, n$ galaxies with $z f g=2$ as $0.85 \times n$, and those with $z f g \geq 3$ remain unchanged.

13 The linear growth factor for the chosen cosmology was obtained from the ICRAR's Cosmology Calculator (https://cosmocalc. icrar.org/). 
Table 8. G237 structures SFR.

\begin{tabular}{|c|c|c|c|}
\hline Members & $\mathrm{N}$ & $\begin{array}{c}\mathrm{SFR} \\
\left(M_{\odot} \mathrm{yr}^{-1}\right)\end{array}$ & $\begin{array}{c}\text { SFRD } \\
\left(M_{\odot} \mathrm{yr}^{-1} \mathrm{cMpc}^{-3}\right)\end{array}$ \\
\hline Full: $2.15 \leq z_{\mathrm{spec}}<2.20$ & 31 & $1485 \pm 71$ & $0.080 \pm 0.004$ \\
\hline ss $1: 2.15 \leq z_{\mathrm{spec}} \leq 2.164$ & 20 & $1002 \pm 58$ & $0.229 \pm 0.013$ \\
\hline ss2: $2.19 \leq z_{\text {spec }} \leq 2.20$ & 8 & $429 \pm 41$ & $0.115 \pm 0.011$ \\
\hline $\operatorname{HAE}^{(a)}\left(\mathrm{w} / \mathrm{o} z_{\text {spec }}\right.$ members $)$ & 32 & 715 & 0.163 \\
\hline Full+HAE & 63 & $2200 \pm 71$ & $0.119 \pm 0.004$ \\
\hline ss $1+\mathrm{HAE}$ & 52 & $1717 \pm 58$ & $0.392 \pm 0.013$ \\
\hline SPIRE: $1.8<z_{\text {phot }}<2.5$ & 11 & $2408_{-106}^{+134}$ & $0.130_{-0.006}^{+0.007}$ \\
\hline Full+HAE+SPIRE ${ }^{(b)}$ & 70 & $3995_{-128}^{+150}$ & $0.216_{-0.007}^{+0.008}$ \\
\hline Core $^{(c)}$ & 6 & $436 \pm 41$ & $9.6 \pm 0.9$ \\
\hline
\end{tabular}

Notes. ${ }^{(a)}$ The HAE sample contains 38 sources, of which six are also present in ss1 and in the full spectroscopic samples, their SFRs are thus not considered in this subsample. We note that the HAE SFRs are derived from the extinction-corrected $\mathrm{H} \alpha$ luminosity (Koyama et al 2021). ${ }^{(b)}$ Four SPIRE sources are already included in the full spectroscopic sample and are thus not considered in the SPIRE subsample for the total SFR estimate. ${ }^{(c)}$ We consider the core the A component with four spectroscopic members, of which three are HAEs, and two additional HAEs. The volume of the core is $45 \mathrm{cMpc}^{3}$, corresponding to $0.8^{\prime} \times 1.1^{\prime}=1.45 \times 1.89 \mathrm{cMpc}^{2}$, and a transverse distance of $16.4 \mathrm{cMpc}$, corresponding to $\Delta z=2.1637-2.1517$.

Cucciati et al. 2018). The derived $\delta_{\mathrm{L}}(z=0)$ values are reported in Table 7. The derived $\delta_{\mathrm{L}}(z=0)$ exceeds the collapse threshold of $\delta_{\mathrm{c}}=1.686$ for the two substructures, and it does not for the full structure. Therefore, both substructures are likely to virialize independently and form a larger, but likely not virialized structure. The $z=0$ mass of the two substructures are comparable to the mass of a Virgo-type cluster (i.e., $\sim(3-10) \times 10^{14} M_{\odot}$; Fouqué et al. 2001).

\subsection{G237 contribution to the cosmic SFR density}

The total SFRs of the structures in G237, obtained by adding the SFR of all spectroscopic members, are $1485 \pm 71 M_{\odot} \mathrm{yr}^{-1}$ if we consider the full structure, $1002 \pm 58 M_{\odot} \mathrm{yr}^{-1}$ for $\mathrm{ss} 1$, and $429 \pm 41 M_{\odot} \mathrm{yr}^{-1}$ for ss2. If we consider the HAEs presented in Koyama et al. (2021) and their SFRs derived from the dust-corrected $\mathrm{H} \alpha$ luminosity, we have an additional SFR of $715 M_{\odot} \mathrm{yr}^{-1}$. This estimate does not include the SFR from the six sources in common between the two samples. Including the SFR from the HAEs, the total SFRs become $2200 \pm 71 M_{\odot} \mathrm{yr}^{-1}$, and $1717 \pm 58 M_{\odot} \mathrm{yr}^{-1}$, for the full structure, and for ss 1 , respectively. The estimated SFRs include the four spectroscopically confirmed members detected in the sub-mm by SPIRE. These are relatively faint sub-mm sources, with $S_{350 \mu \mathrm{m}}<18 \mathrm{mJy}$, and SFR 110-220 $M_{\odot} \mathrm{yr}^{-1}$. Since not all SFG members in the structure have been identified, even after considering the HAEs, the estimated total SFRs are only a lower limit. We note that the HAE selection is limited to $z<2.17$, and to a $7^{\prime} \times 4^{\prime}$ region. In addition, some heavily extincted SFGs might not be detectable as HAEs, and a large fraction of the field, where the density of SPIRE source peaks, is affected by a bright star at optical and NIR wavelengths. We estimate how much SFR we might be missing because of dust extinction and of the masked area, by considering the 11 SPIRE member candidates, minus the 4 already included in the spectroscopic sample (see Sect. 4.3). The total SFR, including these additional seven (11 candidates, minus four confirmed), increases to $\sim 4000 M_{\odot} \mathrm{yr}^{-1}$. We also derive the SFR in the protocluster core by adding the SFRs of the four spectroscopic members, and two additional HAEs located in the A component. All the derived SFRs, and inferred SFR densities (SFRDs) are listed in Table 8.

Compared to the average cosmic SFR density (SFRD) at $z \sim 2.16\left(\langle\operatorname{SFRD}(z=2.168)\rangle=0.128 M_{\odot} \mathrm{yr}^{-1} \mathrm{Mpc}^{-3}\right.$; Madau \& Dickinson 2014), the SFRD of ss 1 and of the core are, respectively, $3.1 \pm 0.1$ and $75 \pm 7$ times higher, while the full structure SFRD is consistent or $\sim 1.6$ times higher if the SPIRE member candidates are included. Since the full structure includes a much bigger volume than the two substructures, it is not surprising that its SFRD is closer to the average cosmic value.

It is interesting to note that the enhanced SFRD in the core with respect to the rest of the structure is mainly due to the larger galaxy density $\left(0.13\right.$ spectroscopic members and HAEs $\mathrm{cMpc}^{-3}$ in the core versus $0.01 \mathrm{cMpc}^{-3}$ in ss1), and, only in part due to a larger average SFR per galaxy in the core than in the full structure, $\langle\mathrm{SFR}\rangle=73 \pm 52 M_{\odot} \mathrm{yr}^{-1}$ in the core versus $35 \pm 27 M_{\odot} \mathrm{yr}^{-1}$ in the full sample. The difference in average SFR is, however, less important than the number density, and not highly significant as the SFRs span a wide range, and suffer from large uncertainties.

Since ss1, and its core are the most significant structures in the G237 field, we compare their SFRD estimates with those of other protoclusters from the literature at similar redshifts (Clements et al. 2014; Dannerbauer et al. 2014; Kato et al. 2016), and with theoretical models (Chiang et al. 2017). Chiang et al. (2017) predict that the contribution from protoclusters, and protocluster cores to the cosmic SFRD at $z \sim 2.2$ is $12-17 \%$, and $1.2-2.9 \%$, respectively. These estimates are obtained using two semi-analytical simulations (SAM; Henriques et al. 2015; Guo et al. 2013), and are based on all galaxies with $\mathcal{M}>10^{8.5} M_{\odot} \mathrm{yr}^{-1}$. Based on these simulations, the predicted SFRDs for an average protocluster, and its core at $z \simeq 2$, are $\sim 0.6 M_{\odot} \mathrm{yr}^{-1} \mathrm{Mpc}^{-3}$, and 61-91 $M_{\odot} \mathrm{yr}^{-1} \mathrm{Mpc}^{-3}$, respectively (Chiang et al. 2017). These estimates assume a spherical volume with average radius of $8 \mathrm{cMpc}$, and $0.8 \mathrm{cMpc}$, for the protocluster and its core, corresponding to $\sim 8^{\prime}$, and $0.8^{\prime}$, respectively at our redshift. Our estimate of SFRD in the core is almost one order of magnitude smaller than the average value predicted by the models, but the volume we consider is $>20$ times bigger. The SFRD relative to ss1 (i.e., $0.39 \pm 0.01 M_{\odot} \mathrm{yr}^{-1} \mathrm{Mpc}^{-3}$ ) is also lower than predicted, but only by a factor of 1.6, and the considered volume is twice than in the model. The smaller SFRDs with respect to the model predictions can be in part explained by having considered bigger volumes, and possibly by not having included all star-forming members. Overall, we can conclude that the SFRD estimates derived for the ss1, and for the core can be reconciled with the predictions from theoretical models (Chiang et al. 2017).

Compared to the SFRD estimates of other protoclusters at similar redshift from the literature (i.e., 500$5000 M_{\odot} \mathrm{yr}^{-1} \mathrm{Mpc}^{-3}$; Clements et al. 2014; Dannerbauer et al. 2014; Kato et al. 2016), the SFRDs in ss 1 or in the core are much lower. There are several differences between these works and ours that should be taken into account before making any comparison. In these works, the considered volume is often assumed to be a sphere of diameter equivalent to the members projected distance, and physical dimensions are considered rather than comoving, which yield smaller volumes, and thus larger SFRDs by a factor of $>10-15$ at $z \sim 2-2.5$ (see e.g., Dannerbauer et al. 2017). In some cases, the integrated SFR is obtained considering all sub-mm detected galaxies, even when membership is 
not spectroscopically confirmed (see e.g., Clements et al. 2014; Kato et al. 2016). Finally, their SFR estimates are systematically higher than ours by a factor of 1.7-1.8 because they assume a Salpeter (1955) IMF and the L(IR)-SFR relation reported in Bell (2003). Because of all these differences we will not carry out a comparison with the SFRDs reported in the literature for other protoclusters and warn the reader against using those estimates to identify extreme sources and rule out models.

\subsection{Quenching signs and the role of the AGN}

Protocluster first descendants, clusters at $z \sim 1.5-2$, are already dominated by a population of massive quiescent galaxies in excess compared to the field (Andreon et al. 2014, 2016; Strazzullo et al. 2019). We can thus expect these galaxies to start quenching during the protocluster phase. Our protocluster, $\mathrm{G} 237$ at $z=2.16$, can give us a first glance at this elusive quenching population, and at the processes responsible for halting their star formation. Theoretical studies often invoke AGN feedback as one of the main quenching mechanisms, especially in the most massive galaxies (e.g., Rosito et al. 2021). AGN feedback can occur mechanically, via a radio jet (radio mode), or radiatively (quasar mode), through an AGN-driven wind. The radio mode corresponds to AGN accreting well below the Eddington rate (Eddington ratio $\lambda_{\text {Edd }}<0.01$ ) and with a radio jet. The quasar mode instead corresponds to high luminosity AGN that accrete at near their Eddington ratio and are capable of driving strong winds (Harrison 2017).

Out of the four members hosting AGN activity, three have relatively modest stellar masses, $\sim(2-5) \times 10^{10} M_{\odot}$, and large SFRs, $\sim 50-140 M_{\odot} \mathrm{yr}^{-1}$. The fourth AGN, ID SL01, is instead just below the $\mathrm{MS}$ and, with $\mathcal{M} \simeq 1.3 \times 10^{11} M_{\odot}$ and $\mathrm{SFR} \sim 34 M_{\odot} \mathrm{yr}^{-1}$, turns out to be more massive and less active than the other AGN members. This source is in the structure core and might be at the end of its growing phase and starting to quench. The rest-frame optical spectrum of this source shows a slightly broad $\mathrm{H} \alpha$ line, suggesting that the AGN is unobscured. Its X-ray emission is weak, visible in the Chandra image, but not detected with a high enough signal-to-noise to be included in the Cosmos Chandra released catalog. These properties are consistent with the AGN evolutionary scenario where, following AGN feedback, the line of sight is cleared out from the obscuring dust, and, both the SF activity and AGN luminosity, decrease due to lack of fuel (Sanders et al. 1988). ID SL01 is not detected in the deep radio images available in the field, we can thus rule out the presence of a powerful radio jet. To investigate instead whether this source is radiatively powerful enough to launch a wind that can halt the current star formation, we estimate its Eddington ratio. This value is derived from the ratio of the SED-derived bolometric luminosity, and the Eddington luminosity that depends on the black hole $(\mathrm{BH})$ mass. Based on the optical luminosity at $5100 \AA$ in the rest-frame (i.e., $\left.\log \left(\lambda L_{\lambda}(5100 \AA) /\left(\mathrm{erg} \mathrm{s}^{-1}\right)\right)=44.6\right)$ and on the width of the $\mathrm{H} \alpha$ line (i.e., $\left.\mathrm{FWHM}\left(\mathrm{H} \alpha^{\text {broad }}\right)=4004 \mathrm{~km} \mathrm{~s}^{-1}\right)$, we estimate a BH mass in SL01 of $\simeq 2.0 \times 10^{8} M_{\odot}{ }^{14}$.

The estimated bolometric luminosity, derived from the CIGALE best fit model, and assuming that is solely due to the AGN, is $2.9 \times 10^{45} \mathrm{erg} \mathrm{s}^{-1}$. Since the AGN does not dominate the bolometric emission in SL01, as its SED clearly shows (see Fig. E.1), the SED-derived bolometric luminosity should be considered as an upper limit to the AGN bolometric luminosity. The

\footnotetext{
${ }^{14} M_{\mathrm{BH}} / M_{\odot}=4.817 \times\left[\lambda L_{\lambda}(5100 \AA) / 10^{44} \mathrm{erg} \mathrm{s}^{-1}\right]^{0.7} \times F H W M(\mathrm{H} \alpha)^{2}$ where $F H W M(\mathrm{H} \alpha)$ is in $\mathrm{km} \mathrm{s}^{-1}$ (Woo \& Urry 2002).
}

derived upper limit to its Eddington ratio, $\lambda_{\text {Edd }}=L_{\text {bol }} / L_{\text {Edd }}<$ 0.11 , is high enough to cause some feedback onto the host galaxy through the AGN radiation.

We can investigate whether the other AGN members in the structure could cause AGN feedback. None of them is detected in the radio, thus we can rule out the radio mode feedback. To estimate their Eddington ratio, we use the bolometric correction as this quantity is correlated with the Eddington ratio (Lusso et al. 2012). The bolometric correction $k_{\text {bol }}$, can be estimated from the ratio between the AGN bolometric luminosity and the $\mathrm{X}$-ray luminosity, using $k_{\mathrm{bol}}=L_{\mathrm{bol}} / L_{\mathrm{X}}$. We derive $L_{\mathrm{bol}}$ from the best fit SED, it is thus an upper limit to the AGN bolometric luminosity. $L_{\mathrm{X}}$ is derived from the $0.5-10 \mathrm{kev}$ measured flux, and it is a lower limit since it is not corrected for absorption. The estimated upper limits to the $k_{\text {bol }}$ values are thus $<57,95$, and 26, for IDs 55326, 58057, and L710971, respectively. Assuming the relation between $\lambda_{\text {Edd }}$, and $k_{\text {bol }}$ for unobscured AGN reported by Lusso et al. (2012), the ensuing $\lambda_{\text {Edd }}$ would be $\lesssim 0.3,0.6$, and 0.1 , for IDs 55326, 58057, and L710971, respectively. The estimated Eddington ratios imply that these AGN can produce a significant radiative feedback.

The high AGN fraction in G237 suggests a connection between such an activity and the protocluster environment. Overall, all AGN members are powerful enough to exert some radiative feedback, and this might be quenching the star formation activity in ID SL01, a massive galaxy hosting an AGN with a lower SFR than expected for a MS galaxy with the same mass (see Fig. 16).

\subsection{Structure properties that make a Planck high-z source}

The overall SFR of G237 is not extreme, its members are ordinary SFGs, and the protocluster overall properties are similar to those observed in other protoclusters. It is thus legitimate to ask why G237 is significantly detected by Planck, while most protoclusters from the literature are not. To investigate whether there is a specific property that explains its Planck significant detection, we compare G237 with other protoclusters situated in the Cosmos field at $z \sim 2-2.5$. There are other protoclusters found at much higher redshifts (e.g., Capak et al. 2011; Pavesi et al. 2018; Lemaux et al. 2018; Mitsuhashi et al. 2021), but we do not analyze them as the Planck high- $z$ source selection is not tailored to find protoclusters at $z \gtrsim 4$.

To our knowledge there are no other known structures in the Cosmos field at exactly the same redshift as G237. G237 is located to the east of the central $1 \mathrm{deg}^{2}$ of the Cosmos field, where many deep and extensive sub-mm and spectroscopic surveys have been carried out. A bright star, situated in the field and covering a circular region of 2.4-3.6' in diameter (depending on the band), prevents the detection of sources in the optical and NIR that are thus missing in the Cosmos L16 multiband cata$\log$. These considerations might in part explain why this structure was not previously discovered through spectroscopic or high precision photometric redshifts. It is also possible that the nature of G237 and of the PHz sources, in general, is different from those discovered through other techniques.

There are two known structures in Cosmos that are also bright at sub-mm wavelengths, PCL1002 at $z=2.47$ (with 41 spectroscopically confirmed members, including seven SMGs, one QSO and 33 LBGs, over $20^{\prime} \times 20^{\prime}$; Casey et al. 2015) and CLJ1001 at $z=2.51$ (with 24 spectroscopically confirmed members, including $1 \mathrm{SMG}$, and six HAEs, over $\sim 3^{\prime} \times 3^{\prime}$; Wang et al. 2016, 2018). These structures are considered part of a proto-supercluster at $z=2.45$ 


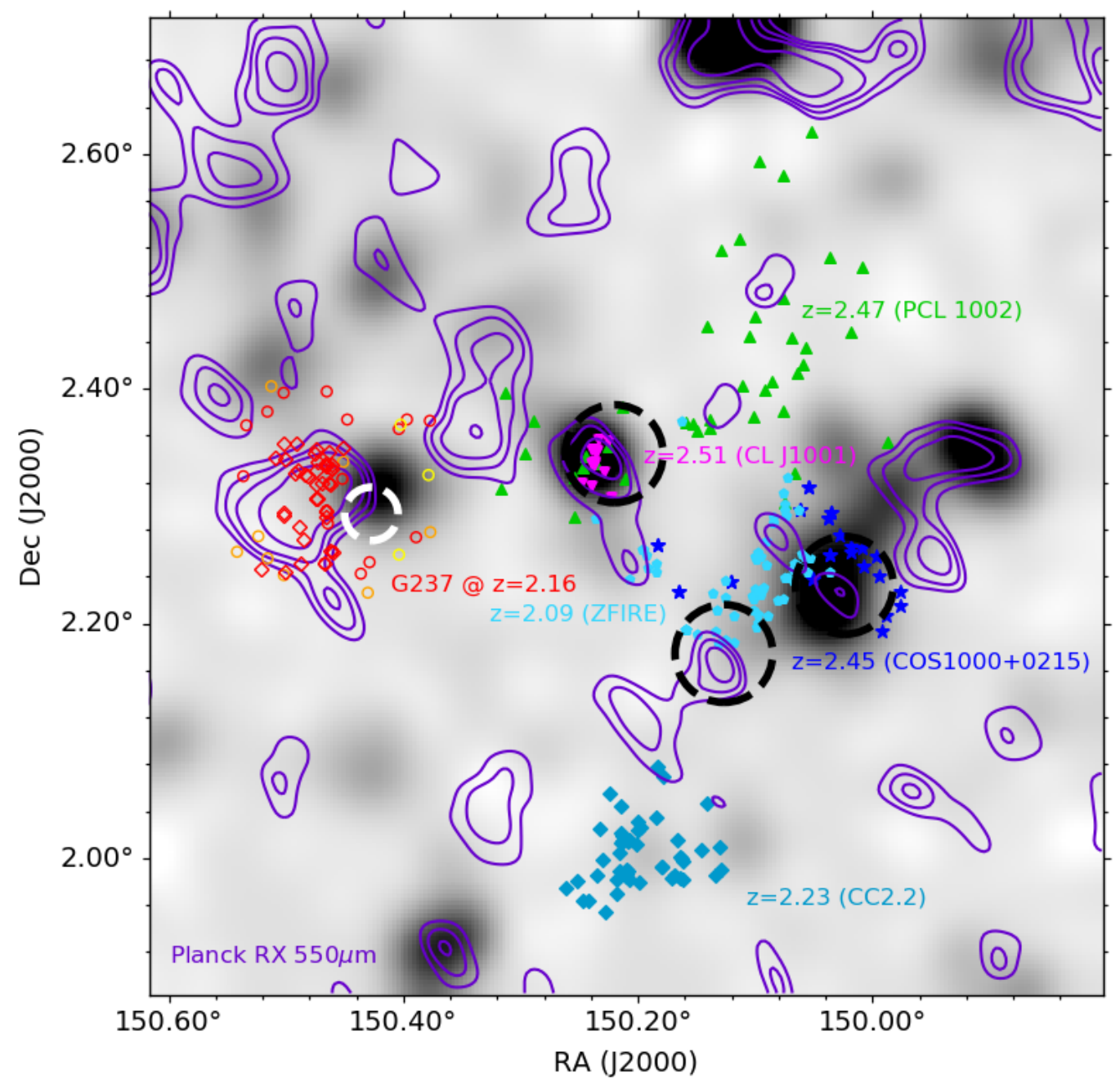

Fig. 18. Layout of the structures found in a $50^{\prime} \times 50^{\prime}$ region in the Cosmos field overlaid on a density map of red SPIRE sources. Planck red-excess contours (at 30,40, 50, 68, 95 and 99.7\% of the highest value) are shown as purple lines. Different symbols are members of the following structures: COS1000+0215 protocluster at $z=2.45$ (blue stars; Diener et al. 2015; Chiang et al. 2015), CL J1001 at $z=2.51$ (magenta up-side down triangles; Wang et al. 2016, 2018), PCL 1002 at $z=2.47$ (green triangles; Casey et al. 2015), CC2.2 at $z=2.23$ (turquoise blue diamonds; Darvish et al. 2020), and ZFIRE at $z=2.09$ (light blue circles; Yuan et al. 2014; Hung et al. 2016). The spectroscopic members in G237 are shown as open circles (red: $2.15 \leq z<2.164$, yellow: $2.164<z<2.19$, and orange: $2.19<z<2.20$ ), and the HAEs (Koyama et al. 2021) as red diamonds. The dashed white circle represents the 1.4' radius circle masked in the L16 Cosmos catalog due to a bright star. Large black dashed circles ( $2.5^{\prime}$ radius) indicate locations, close to the selected protoclusters, where a signal $\gtrsim 2 \sigma$ is detected in the Planck RX map, but not significant enough to be selected as a $\mathrm{PHz}$ source.

(Cucciati et al. 2018) that includes 96 spectroscopically confirmed members, initially discovered as an overdensity of LAEs over a region of $1 \times 1 \operatorname{arcmin}^{2}(\operatorname{COS} 1000+0215$; Diener et al. 2015; Chiang et al. 2015; Civano et al. 2012; Kriek et al. 2015; Trump et al. 2009; Casey et al. 2015). There are two other significant structures in the Cosmos field at $z \simeq 2, \mathrm{CC} 2.2$ at $z=2.23$, discovered as overdensity of HAEs (Darvish et al. 2020), and ZFIRE at $z=2.085$, discovered in the Z-FOURGE photometric redshift survey (Spitler et al. 2012; Yuan et al. 2014; Hung et al. 2016). The main properties of these protoclusters are listed in Table 9, and the locations of their confirmed members are shown in Fig. 18.

All these structures have estimated total SFRs larger than what we measure in $\mathrm{G} 237$, and are massive structures $\left(M_{\mathrm{h}}(z=\right.$ $\left.0) \simeq 3-80 \times 10^{14} M_{\odot}\right)$. However, none of them is detected as a Planck high- $z$ source. In Fig. 18, we show the density map of red SPIRE sources in a $50^{\prime} \times 50^{\prime}$ region where all above structures are located, and the contours of the Planck RX map at $550 \mu \mathrm{m}$.
The figure shows that the Planck RX map detects the overdensities of red SPIRE sources. The red SPIRE sources overdensity in G237 is slightly off the Planck RX peak. We note that the bright star in the G237 field is close to the position of the SPIRE overdensity, thus no galaxy can be investigated in that region in the optical/NIR. There are three Planck sources detected at $\sim 2$ $3 \sigma$ level in the Planck maps at the position of CL J1001, where some members of the PCL 1002 structure are also present, and close to COS1000+0215, and ZFIRE (see black dashed circles in Fig. 18). The association with these structures is probably due to the presence of bright SPIRE sources that belong to these two structures. The sub-mm signal in the vicinity of COS $1000+0215$ might indicate that the structure is more extended and include DSFGs that are not detectable as LAE. Emission line galaxies (e.g., LAEs, and HAEs) are rarely detected at sub-mm wavelengths, and there is some evidence that they might occupy different regions than DSFGs in protoclusters (see e.g., Umehata et al. 2015, 2017; Lee et al. 2017). Interestingly, there are nine DSFGs 
in the ZFIRE protocluster, but this structure is not detected by Planck. A possible explanation is that the DSFG members are not sufficiently clustered. Indeed they have been found by a dedicated search of DSFGs with spectroscopic redshift in the region around ZFIRE (Hung et al. 2016). On the contrary, in G237 two out of four DSFGs are in the core, and are also detected as HAEs.

This analysis indicates that the total SFR, the halo mass, and the extension do not determine whether a protocluster will be detected by Planck and selected as a PHz. The main key factor is possibly the presence of a large concentration of DSFGs at $z \sim 2$, but this can be also due to projection effects or to amplification of line-of-sight structures. We would need to analyze more $\mathrm{PHz}$ sources to confirm such a claim.

This comparison also highlights the importance of understanding the connection between protoclusters identified through different selection techniques, and the different types of member galaxies (massive galaxies, LAEs, HAEs, DSFGs, and AGN) and how these vary as a function of halo mass, and redshift. We can expect that galaxies of a certain type are mostly associated with a specific evolutionary stage, for example sub-mm galaxies tend to be more massive, and thus in a more advanced phase than LAEs. Protoclusters found through different galaxy types might then be at different maturity levels. These questions can only be addressed by studying a large number of protoclusters covering a significant range of redshifts, masses, and selections, but this would be a highly demanding endeavor due to the need of multiwavelength deep data over wide areas.

The lack of starbursting members in G237 is hard to reconcile with the Planck selection, which was intended to find such systems. Interestingly, based on the protocluster cosmic filling factor (i.e., 0.02 at $z \sim 2$; Chiang et al. 2017), protoclusters are expected to occupy a volume of $\sim 1500 \mathrm{cMpc}^{3}$ in a random sightline in a $\sim 10^{\prime}$ field over $2.0<z<2.2$. The two substructures in G237 occupy, combined, a much larger volume, that is $\sim 8100 \mathrm{cMpc}^{3}$. Thus, having found these two protoclusters in a Planck source field is not fortuitous, and the $5 \sigma$ overdensity of red SPIRE sources (see Sect. 4.2) is likely associated with the structures. Starbursting protoclusters or with a large number of DSFGs at $z \lesssim 3$ are expected to be in the Planck source list, and the best way to find them might be choosing those with the most significant sub-mm overdensities. G237 is among the least overdense Planck sources, and this might explain the low level of starbursting activity, but a study of another Planck source (i.e., PLCK G073.4-57.5; Kneissl et al. 2019, Hill, priv. comm.), characterized by a large overdensity also reveals a dominance of normal SFG members, rather than starbursts, and a large number of DSFG members (five detected by SPIRE, and three by SCUBA2).

\subsection{Physical processes that power the star formation activity in G237}

The analysis of the member properties with respect to their location in G237 shows a weak dependence on the environment (see Sect. 5.4). Although most spectroscopic members are blue SFGs, those in the core are on average more massive and more active. Also, the fraction of DSFGs and AGN is much higher in the core (i.e., $f_{\mathrm{AGN}}^{\text {core }}=f_{\mathrm{DSFG}}^{\text {core }}=50 \%$ versus $f_{\mathrm{AGN}}^{\text {full }}=f_{\mathrm{DSFG}}^{\text {full }}=13 \%$ ). The ubiquity of both SF (traced by the sub-mm emission), and AGN (traced by the X-ray emission) activity suggests a common mechanism at their origin. Both SF and AGN require gas to fuel them, and their enhancement in the core suggests that the fueling mechanism might be favored in dense regions. Gas can be funneled into the center of galaxies through gas-rich mergers or through gravitational disk instabilities caused by inflowing gas (e.g., Kereš et al. 2005, 2009; Dekel \& Birnboim 2006; Dekel et al. 2009).

In Sect. 5.6, we find morphologies consistent with signs of interaction or disturbance in about $40 \%$ of the members, and $\sim 20 \%$ have also a close companion (see Table 6 and Figs. D.1-D.3). These properties are consistent with various merger phases, from pre-merging (pairs at $\lesssim 3^{\prime \prime}$ at our redshift; Ventou et al. 2017), to ongoing or post merging events. Since close encounters and interactions are more likely in overdensities (Jian et al. 2012), mergers might be a viable fueling mechanism. However, the lack of a clear trend between the morphology and the spatial distribution questions such an interpretation. High spatial resolution imaging in the optical rest-frame, or kinematic measurements of the gas in these systems would be necessary to determine the role of mergers.

Alternatively, cold gas streams can also be a viable fueling channel in G237 members. Cold gas flows are expected to fuel SF in massive halos only at high redshifts. Below a critical redshift, $z_{\text {crit }} \gtrsim 2$, the streaming cold gas is shock-heated in massive halos $\left(M_{\mathrm{h}} \gtrsim 10^{12} M_{\odot}\right)$, and does not readily fuel ongoing SF. G237 is hosted by a massive halo and it is at a redshift below $z_{\text {crit }}$. The same is true for the other protoclusters in the Cosmos field listed in Table 9. In Fig. 19, we place the selected protoclusters on a halo mass versus redshift diagram, and compare them with the different gas regimes proposed by Dekel \& Birnboim (2006). The halo mass and redshift marking the transition between the cold streams to the shock-heated regime are not yet well constrained, theoretically, nor observationally. Here, we show the values from Dekel \& Birnboim (2006) (but see also Lapiner et al. 2021). To place the selected protoclusters in the diagram, we estimate their halo mass at the observed redshift. Those estimates were obtained by taking their estimated $z=0$ halo mass and evolving it back in time using the halo accretion growth based on fitting functions of Behroozi et al. (2013) (see dashed lines in Fig. 19), when not available in the literature ${ }^{15}$.

All selected protoclusters are in the shock-heated regime implying that cold gas accretion should be inhibited and starformation will continue as long as there is cold gas, and might end by starvation. Assuming a constant SFR equal to the average value in ss1 (i.e., $33 M_{\odot} \mathrm{yr}^{-1}$ ), and the relation between SFR and cold molecular gas for normal SFGs as formulated by Sargent et al. (2014), these galaxies have, on average, $\left\langle M_{\mathrm{mol}}\right\rangle \sim$ $3.4 \times 10^{10} M_{\odot}$. If we assume that all the molecular gas will be converted into stars, this molecular reservoir will be depleted in $\sim 1 \mathrm{Gyr}$, or at $z \simeq 1$.6. This simple scenario implies no need for cold gas accretion or mergers to sustain the current SFR in our protocluster members, but does not explain the SFR enhancement in the core.

\subsection{Member properties in G237 and in other protoclusters}

In Sect. 5.4, we find that most members in G237 are on the MS, and no starburst members have been found even among the SPIRE-detected sources. Here, we compare G237 member properties with those of other protoclusters at $z \sim 2-3$ with spectroscopically confirmed members and available SFRs, and

${ }^{15}$ For PCL 1002, and CL J1001, we used the estimated halo mass at the observed redshift reported by Casey et al. (2015), and Wang et al. (2016) (i.e., $(8 \pm 3) \times 10^{14} M_{\odot}$ for PCL 1002 , and $\left(7.9_{-2.9}^{+4.7}\right) \times 10^{13} M_{\odot}$ for CL J1001). 
Table 9. Protoclusters in the Cosmos field at $2.0<z<2.5$.

\begin{tabular}{llccccrr}
\hline \hline Name & $\begin{array}{l}\text { Redshift } \\
{\left[z_{\min }-z_{\max }\right]}\end{array}$ & $\begin{array}{c}N \text { spec. } \\
\text { members }\end{array}$ & $\begin{array}{c}M_{\mathrm{h}}(z=0) \\
\left(10^{14} M_{\odot}\right)\end{array}$ & $\begin{array}{c}\Sigma \text { SFR } \\
\left(M_{\odot} \mathrm{yr}^{-1}\right)\end{array}$ & $\begin{array}{c}\text { Extent } \\
(\operatorname{arcmin})\end{array}$ & $\begin{array}{c}\text { N. DSFG }(a) \\
\& \text { AGN }\end{array}$ & $\begin{array}{c}\text { Reference } \\
\text { ZFIRE }\end{array}$ \\
$2.085[2.076-2.104]$ & 57 & $2.5_{-1.2}^{+2.5}$ & 4002 & $7.4 \times 10$ & $9 \& 4$ & $(1,2)$ \\
CC2.2 & $2.233[2.122-2.247]$ & 47 & 9.2 & 2228 & $9.2 \times 9.2$ & $0 \& 3$ & $(3)$ \\
COS1000+0215 & $2.444[2.428-2.456]$ & 20 & $3.2_{-1.9}^{+4.8}$ & $\ldots$ & $5.0 \times 3.1$ & $0 \& 4$ & $(4,5)$ \\
PCL 1002 & $2.472[2.463-2.487]$ & 41 & $77_{-41}^{+53}(b)$ & 4447 & $3.0 \times 1.5$ & $7 \& 5$ & $(6)$ \\
CL J1001 & $2.507[2.494-2.515]$ & 18 & $83_{-44}^{+96}(b)$ & 2790 & $2.7 \times 3.0$ & $1 \& 1$ & $(7,8)$ \\
\hline G237 ss1 & $2.155[2.150-2.164]$ & 20 & $6.6_{-3.5}^{+26}$ & $1002 \pm 58$ & $9.5 \times 9.3$ & $3 \& 4$ & $($ This work) \\
G237 ss2 & $2.195[2.190-2.199]$ & 8 & $5.1_{-1.9}^{+20}$ & $429 \pm 41$ & $9.9 \times 10.6$ & $1 \& 0$ & (This work) \\
\hline
\end{tabular}

Notes. ${ }^{(a)}$ Here, we consider a DSFG a galaxy detected in the sub-mm by SPIRE, SCUBA-2, LABOCA, or AzTEC, even if it might comprise multiple galaxies as in the case of CL J1001 where ALMA finds five sources associated with a single SPIRE source (Wang et al. 2016). ${ }^{(b)}$ For PCL 1002, and CL J1001, the $z=0$ halo masses have been derived from the estimated halo mass at the observed redshift (Casey et al. 2015; Wang et al. 2016), these are $(8 \pm 3) \times 10^{13} M_{\odot}$ for PCL 1002, and $\left(7.9_{-2.9}^{+4.7}\right) \times 10^{13} M_{\odot}$ for CL J1001, and assuming the mass growth as formulated by Behroozi et al. (2013), to be consistent with the other structures analyzed here. These are larger than those derived by Casey et al. (2015), and Wang et al. (2016) assuming a mass-dependent exponential growth model (i.e., $(2 \pm 1) \times 10^{15} M_{\odot}$ ).

References. (1) Yuan et al. (2014), (2) Hung et al. (2016), (3) Darvish et al. (2020), (4) Diener et al. (2015), (5) Chiang et al. (2015) (6) Casey et al. (2015), (7) Wang et al. (2016), (8) Wang et al. (2018).

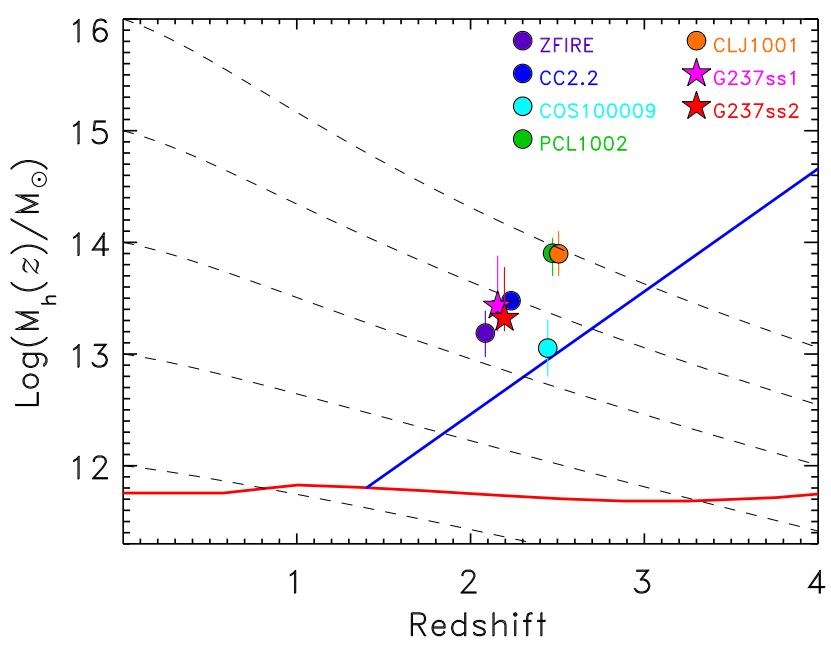

Fig. 19. Protocluster dark matter halo mass as a function of redshift for the protoclusters in the Cosmos field at $z \simeq 2$ listed in Table 9 as annotated, and for the G237 substructures (ss1: magenta full star, ss2: red full star). The dashed black lines represent the average halo mass growth based on the fitting functions formulated by Behroozi et al. (2013). Thick lines indicate different gas cooling regimes as predicted by Dekel \& Birnboim (2006): below a critical halo mass of $M_{\mathrm{b}}^{\text {crit }} \simeq 10^{12} M_{\odot}$ (red curve) inflowing gas is predominantly cold allowing galaxies to grow (in addition to merging); above $M_{\mathrm{h}}^{\text {crit }}$ (red curve), and below a critical redshift, $z^{\text {crit }}$ (to the left of the blue line), cold gas flows are shockheated, thus shutting off most of the gas supply to the galaxies; above $M_{\mathrm{h}}^{\text {crit }}$ (red curve), and above $z^{\text {crit }}$ (to the right of the blue line) cold gas is able to penetrate the hot gas environment through cold flow streams favoring galaxy growth and star formation.

stellar masses. These protoclusters are drawn from three different selection methods: searches around HzRGs, overdensities of red optical-NIR sources, HAEs, or sub-mm galaxies. The list of selected protoclusters for this comparison is reported in Table 10, along with the number of members with measured SFR and the integrated SFR. The star formation rates are either derived from FIR-sub-mm data corrected for a Chabrier (2003) IMF, from the dust corrected $\mathrm{H} \alpha$ luminosity, or from the SED fitting. The SFR as a function of redshift of single members as well as the integrated SFR of each protocluster are compared with G237 in Fig. 20 (left panel). Single structure members' SFR span a wide range, $\gtrsim 2$ dex. Sources with large SFRs $\left(>100 M_{\odot} \mathrm{yr}^{-1}\right)$ are found in protoclusters drawn from all selection techniques, although the most intense SFGs $\left(>1000 M_{\odot} \mathrm{yr}^{-1}\right)$ are mainly found in sub-mm overdensities, and they are at $z \sim 2.4-2.5$. The spectroscopic members in G237 exhibit a wide range of SFRs, as in other protoclusters at similar redshift. We compare the observed total SFRs with those predicted by empirical models and simulations of protoclusters (Behroozi et al. 2013; Lim et al. 2021) in Fig. 20 and find that the observed ones are higher by a factor of $\sim 30$ on average.

The SFR as a function of stellar mass for all protocluster members for which those estimates are available are shown in the right panel of Fig. 20. The majority of sources lie on the MS. There are several sources above (by a factor of 3) the MS, consistent with being starburst galaxies. They are mostly sub-mm selected members, and massive $\left(>10^{10} M_{\odot}\right)$ galaxies. A few sources are located below the MS, including two members in G237. Members below the MS are present at all stellar masses, they are in MRC 1138-262, in PCL 1002, and in CC2.2, fields where deep observations are available, possibly implying that such members might be present in other structures, but require extensive searches and deep data to be found. We also show the average SFR per stellar mass bin for G237 (full magenta stars), and for the protoclusters at $2.0<z<2.5$ (full green triangles). The upper and lower uncertainty bounds on the average SFRs are estimated by taking the 16th and 84th percentiles. The mean SFRs for G237 and for the protocluster from the literature are both consistent with the MS relation. In G237, the value at the largest masses is below the MS, yet consistent with it if we take into account the associated uncertainty. This offset might hint to a beginning of quenching among the most massive galaxies in G237. It is interesting to explore whether such a population is also present in other protoclusters that exhibit properties of more mature structures, like the presence of a red sequence due to quiescent galaxies, and diffuse X-ray emission (i.e., Cl J1449+0856, and CL J1001; Strazzullo et al. 2016; Valentino et al. 2016; Wang et al. 2016). There is indeed one galaxy in each of these two clusters below the MS, and in the case of $\mathrm{ClJ} 1449+0856$ there are four 
Table 10. Protoclusters from the literature at $1.9<z<2.6$.

\begin{tabular}{lcccrr}
\hline \hline Name & Redshift & $\begin{array}{c}N \\
\text { members }\end{array}$ & $\begin{array}{c}\text { Selection } \\
\text { method }\end{array}$ & $\begin{array}{r}\Sigma \text { SFR } \\
\left(M_{\odot} \text { yr }^{-1}\right)\end{array}$ & Reference \\
\hline CARLA J1018+0530 & 1.952 & 8 & HzRG & 512 & $(1)$ \\
CARLA J0800+4029 & 1.985 & 9 & HzRG & 754 & $(1)$ \\
Cl J1449+0856 & 1.990 & 15 & phot & 1236 & $(2,3)$ \\
CARLA J2039-2514 & 1.999 & 9 & HzRG & 945 & $(1)$ \\
ZFIRE & 2.085 & 9 & phot & 4002 & $(4)$ \\
MRC 1138-262 & 2.157 & 36 & HzRG & 5713 & $(5,6,7)$ \\
PHz G237.01+42.50 ss1 & 2.155 & 20 & sub-mm & 1002 & $($ this work $)$ \\
HELAISS02 & 2.170 & 4 & sub-mm & 1492 & $(8)$ \\
2QZCluster & 2.230 & 7 & sub-mm & 2004 & $(9)$ \\
CC2.2 & 2.233 & 40 & HAE & 2228 & $(10)$ \\
HS 1700+64 & 2.310 & 13 & sub-mm & 5290 & $(9,11,12)$ \\
HATLAS J084933 & 2.412 & 4 & sub-mm & 6026 & $(13)$ \\
PCL 1002 & 2.472 & 41 & sub-mm & 4447 & $(14)$ \\
4C 23.56 & 2.488 & 27 & HzRG & 3887 & $(6,15)$ \\
CL J1001 & 2.507 & 14 & phot & 2790 & $(16)$ \\
USS 1558-003 & 2.528 & 35 & HzRG & 2375 & $(6)$ \\
HXMM20 & 2.609 & 5 & sub-mm & 1877 & $(8)$ \\
\hline
\end{tabular}

Notes. ${ }^{(a)}$ Number of spectroscopically confirmed members for which a SFR estimate is available. ${ }^{(b)}$ The listed protoclusters have been found through the following methods, HzRG: search around a HzRG, phot: identification of an overdensity of photometrically selected high- $z$ sources, sub-mm: overdensity of sub-mm sources, HAE: overdensity of $\mathrm{H} \alpha$ emitters in narrowband images.

References. (1) Noirot et al. (2018), (2) Smith et al. (2019), (3) Valentino et al. (2015), (4) Hung et al. (2016), (5) Dannerbauer et al. (2017), (6) Tadaki et al. (2019), (7) Hatch et al. (2009), (8) Gómez-Guijarro et al. (2019), (9) Kato et al. (2016), (10) Darvish et al. (2020), (11) Lacaille et al. (2019), (12) Chapman et al. (2015), (13) Ivison et al. (2013), (14) Casey et al. (2015), (15) Lee et al. (2017), (16) Wang et al. (2018).
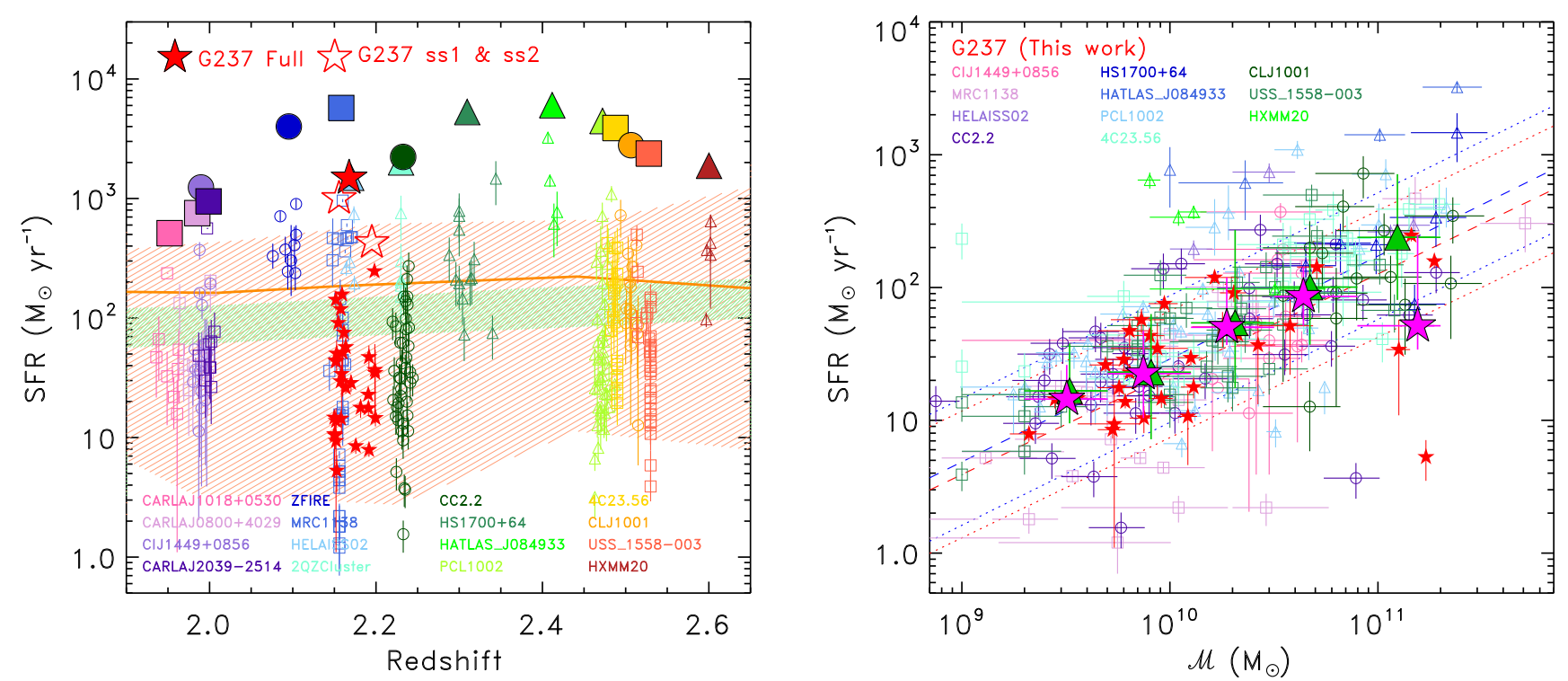

Fig. 20. SFR as a function of redshifts (left panel) and of stellar mass (right panel) of the spectroscopic members in G237 (small filled red stars) and in 16 (11 in the right panel because stellar masses for individual members were not available for all) protoclusters at $1.9 \lesssim z<3$ from the literature (open triangles: sub-mm protoclusters, open circles: color selected (i.e., red optical-NIR sources or HAEs), or open squares: searches around HzRGs). The protocluster names are annotated (for more details see Table 10). Left panel: large symbols represent total SFRs. Predictions from the empirical models of Behroozi et al. (2013) (green hatched region), from the 25 most massive $z=0$ clusters in the TNG300 simulation (salmon hatched area), and the median SFR of the five most massive TNG300 simulated clusters (salmon thick line) (adapted from Lim et al. 2021) are shown for comparison. Right panel: large full green triangles and large full magenta stars are mean SFRs per stellar mass bins for the literature samples with $z<2.5$, and for G237, respectively. Dashed blue and red lines are the MS relation at $z=2$, and 2.6 as parameterized by Speagle et al. (2014), respectively, after correcting it for a Chabrier (2003) IMF. The dotted lines represent the scatter around the MS, equivalent to a factor of 3.

additional members, classified as quiescent galaxies, with only an upper limit to their SFR, that would fall below the MS at high stellar masses $\left(>10^{10.5} M_{\odot} ;\right.$ Strazzullo et al. 2018). These are not shown in Fig. 20 because their SFR estimates are not available. There are, however, other protoclusters, including highly star- forming ones, with several members below the MS, as pointed out earlier. This highlights the heterogeneity observed in protocluster members, which might be in part intrinsic, and in part the result of the variety of techniques, and data used to identify and characterize them. 
In conclusion, the members in G237 exhibit similar SFRs and stellar masses as those observed in other protoclusters at similar redshifts. Overall, the analyzed protoclusters exhibit different fractions, although poorly constrained, of ordinary SFGs, starbursts, and quenching galaxies. These fractions might be related to the protocluster maturity level, but also to observational biases. Deep multiwavelength data, able to identify different types of member galaxies (starburst, SFGs, and quenching) of protoclusters drawn from different selections are necessary to understand the origin of the observed diversity.

\section{Summary and conclusions}

We discuss the spectroscopic identification and characteristics of members of an overdensity at $z \simeq 2.16$ located in a protocluster candidate discovered with the Planck satellite, $\mathrm{PHz}$ G237.01+42.50 (G237). An overdensity of HAEs at the same redshift in this field had been previously reported by Koyama et al. (2021). The overdensity contains 31 spectroscopically confirmed galaxies at $2.15 \leq z_{\text {spec }}<2.20$ over a $10^{\prime} \times 11^{\prime}$ region, consistent with an overdensity significance of $5.4 \sigma$. Within this overdensity, we identify two substructures, ss1 with 20 members at $2.15 \leq z<2.164$, and ss2 with eight members at $2.19 \leq z<2.20$. Both substructures are expected to collapse by $z=0$ and become clusters with $z=0$ halo masses of 5-6 $\times 10^{14} M_{\odot}$, roughly corresponding to the virial mass of a Virgo-type cluster. We thus refer to them as protoclusters. Most members are normal SFGs with a wide range of stellar masses $\left(\mathcal{M}=2 \times 10^{9}-2 \times 10^{11} M_{\odot}\right)$, and are either disks or irregular galaxies with SFRs consistent with the MS relation at their redshift. We find a large AGN fraction of $20 \pm 10 \%$ among the identified members in ss1, selected via the X-ray luminosity or spectral line widths. All AGN members are powerful enough to halt the star formation in their hosts through radiative feedback. However, we do not see any obvious sign of quenching in the AGN members, with one exception where the galaxy is situated below the MS.

We find that the protocluster core, besides being denser, includes members that are, on average, more massive and starforming and contains a larger fraction of AGN and DSFGs than the full sample. Although this result might indicate a more efficient growth in the densest regions within the structure, its significance is not high enough to establish an environmental effect on the growth of these galaxies.

The total SFR estimated by adding the SFR of the spectroscopic members, of the HAEs found in the structure (Koyama et al. 2021), and of the SPIRE member candidates is $\sim 4000 M_{\odot} \mathrm{yr}^{-1}$. Although this is higher than predicted by simulations (Lim et al. 2021), it is much smaller than estimated from the Planck data (i.e., $10173 M_{\odot} \mathrm{yr}^{-1}$ ). By analyzing the Herschel/SPIRE data in the field, in combination with the available ancillary data, we explain this discrepancy as an effect of sources alignment along the line of sight that produces a $5 \sigma$ overdensity of red SPIRE sources in the field. This result is in agreement with the predictions based on the standard scenario for the evolution of large-scale structure (Negrello et al. 2017).

In view of protocluster member selection and identification in future studies, it is important to point out that the LAE selection technique would have missed most of the spectroscopic members analyzed here as they do not exhibit a sufficiently strong Ly $\alpha$ emission line. We plan to use the recently released Cosmic HydrOgen Reionization Unveiled with Subaru (CHORUS) data (Inoue et al. 2020) taken with the NB387 narrowband filter to search for LAEs in this field, and test this hypothesis.
Based on our spectroscopic sample, the HAE selection technique finds $75 \%$ of all the spectroscopic members analyzed here at $z<2.17$, including the DSFGs. Thus, it seems to be an effective technique to find protocluster members with $\mathrm{SFR} \geq 5 \mathrm{M}_{\odot} \mathrm{yr}^{-1}$. However, because of the limited width of the narrowband filters, the narrowband imaging technique only finds members in a narrow redshift range (e.g., $\Delta z \simeq 0.027$ ) and might not sample the full richness of an overdensity.

Further advances in our understanding of these structures might come from observations that will become possible with upcoming facilities. For example, the Euclid (Laureijs et al. 2011) mission will be able to detect all spectroscopic members in G237 in its wide optical-NIR survey, and will reach a $60 \%$ completeness level in detecting structures like G237 ss1 $\left(M_{\mathrm{h}}(z=2) \simeq 5 \times 10^{14} M_{\odot}\right.$; see Fig. 10 in Euclid Collaboration 2019). The James Webb Space Telescope (JWST; Gardner et al. 2006), and ground-based AO imaging observations in the NIR could provide high spatial resolution observations in the optical rest-frame to determine whether mergers are the primary fueling process. Wide field deep NIR spectroscopy with MOONS ${ }^{16}$ on the VLT could provide the identification of a large number of protocluster members and better constrain their stellar population. Deep X-ray observations with Athena (Barret et al. 2020) might detect the proto-ICM hot gas permeating these structures, and probe their halo masses and maturity level. Observations of the molecular gas with IRAM or ALMA would provide secure identification of DSFG members and information on the molecular gas properties (see Polletta et al. 2021). A promising future is ahead for the comprehension of the physical processes occurring in these complex and fascinating objects.

Acknowledgements. We are thankful to the anonymous referee for a careful reading, useful comments and suggestions that improved the quality of this paper. MP kindly thanks F. Cullen for providing the VANDELS stacked spectra in electronic format, and A. Marchetti for reducing the LBT data. MP thanks S. Molendi, S. Andreon, C. Mancini, B. Garilli, A. Gargiulo, L. P. Cassarà, and P. Franzetti for useful discussions. MP acknowledges the financial support from Labex OCEVU. BLF gratefully acknowledges support from the Universite de Paris-Saclay. GV acknowledges financial support from Premiale 2015 MITiC (PI B. Garilli). This work has been carried out thanks to the support of the OCEVU Labex (ANR-11-LABX-0060) and the A*MIDEX project (ANR-11IDEX-0001-02) funded by the "Investissements d'Avenir" French government program managed by the ANR. The work is based on observations obtained with Planck http://www.esa.int/Planck, an ESA science mission with instruments and contributions directly funded by ESA Member States, NASA, and Canada. This research has made use of data from HerMES project (https: //www.cosmos.esa.int/web/herschel/key-programmes). HerMES is a Herschel Key Programme utilising Guaranteed Time from the SPIRE instrument team, ESAC scientists and a mission scientist. The HerMES data was accessed through the Herschel Database in Marseille (HeDaM - http://hedam. lam. $\mathrm{fr}$ ) operated by CeSAM and hosted by the Laboratoire d'Astrophysique de Marseille. This research has made use of the NASA/IPAC Infrared Science Archive, which is funded by the National Aeronautics and Space Administration and operated by the California Institute of Technology. Based on data products from observations made with ESO Telescopes at the La Silla Paranal Observatory under ESO programme ID 179.A-2005 and on data products produced by TERAPIX and the Cambridge Astronomy Survey Unit on behalf of the UltraVISTA consortium. The LBT is an international collaboration among institutions in the United States, Italy and Germany. LBT Corporation partners are: The University of Arizona on behalf of the Arizona university system; Istituto Nazionale di Astrofisica, Italy; LBT Beteiligungsgesellschaft, Germany, representing the Max-Planck Society, the Astrophysical Institute Potsdam, and Heidelberg University; The Ohio State University, and The Research Corporation, on behalf of The University of Notre Dame, University of Minnesota, and University of Virginia. We acknowledge the support from the LBT-Italian Coordination Facility for the execution of observations, data distribution and reduction.

$\overline{16 \text { https://vltmoons.org/ }}$ 
This work is based in part on observations made with the Spitzer Space Telescope, which was operated by the Jet Propulsion Laboratory, California Institute of Technology under a contract with NASA. Based on observations collected at the European Organisation for Astronomical Research in the Southern Hemisphere under ESO programme 175.A-0839. Based in part on data collected at Subaru Telescope, which is operated by the National Astronomical Observatory of Japan. Software: This research made use of astropy, a community developed core Python package for astronomy (Astropy Collaboration 2018), of APLpy, an open-source plotting package for Python (Robitaille \& Bressert 2012), of topcat (Taylor et al. 2005), and of the HEALPix software (Górski et al. 2005). We acknowledge the use of the ICRAR's, and Ned Wright's Cosmology Calculators (https: //cosmocalc .icrar.org/; Wright 2006).

\section{References}

Ageorges, N., Seifert, W., Jütte, M., et al. 2010, Proc. SPIE, 7735, 77351L Alexander, D. M., Bauer, F. E., Chapman, S. C., et al. 2005, ApJ, 632, 736 Altieri, B., \& Dannerbauer, H. 2014, in COSPAR Meeting, 40th COSPAR Scientific Assembly, 40, 73

Andreon, S., Newman, A. B., Trinchieri, G., et al. 2014, A\&A, 565, A120 Andreon, S., Dong, H., \& Raichoor, A. 2016, A\&A, 593, A2 Arnouts, S., Le Floc'h, E., Chevallard, J., et al. 2013, A\&A, 558, A67 Asplund, M., Grevesse, N., Sauval, A. J., \& Scott, P. 2009, ARA\&A, 47, 481 Astropy Collaboration (Price-Whelan, A. M., et al.) 2018, AJ, 156, 123 Barret, D., Decourchelle, A., Fabian, A., et al. 2020, Astron. Nachr., 341, 224 Behroozi, P. S., Marchesini, D., Wechsler, R. H., et al. 2013, ApJ, 777, L10 Bell, E. F. 2003, ApJ, 586, 794

Blain, A. W., Chapman, S. C., Smail, I., \& Ivison, R. 2004, ApJ, 611, 725

Boquien, M., Burgarella, D., Roehlly, Y., et al. 2019, A\&A, 622, A103

Boylan-Kolchin, M., Springel, V., White, S. D. M., Jenkins, A., \& Lemson, G 2009, MNRAS, 398, 1150

Bruzual, A. G., \& Charlot, S. 2003, MNRAS, 344, 1000

Buschkamp, P., Seifert, W., Polsterer, K., et al. 2012, Proc. SPIE, 8446, 84465L Cañameras, R., Nesvadba, N. P. H., Guery, D., et al. 2015, A\&A, 581, A105

Calabrò, A., Castellano, M., Pentericci, L., et al. 2021, A\&A, 646, A39 Calzetti, D., Armus, L., Bohlin, R. C., et al. 2000, ApJ, 533, 682

Capak, P. L., Riechers, D., Scoville, N. Z., et al. 2011, Nature, 470, 233 Cappelluti, N., Brusa, M., Hasinger, G., et al. 2009, A\&A, 497, 635 Cardelli, J. A., Clayton, G. C., \& Mathis, J. S. 1989, ApJ, 345, 245

Casey, C. M. 2012, MNRAS, 425, 3094

Casey, C. M. 2016, ApJ, 824, 36

Casey, C. M., Narayanan, D., \& Cooray, A. 2014, Phys. Rep., 541, 45

Casey, C. M., Cooray, A., Capak, P., et al. 2015, ApJ, 808, L33

Casey, C. M., Cooray, A., Killi, M., et al. 2017, ApJ, 840, 101

Cassata, P., Guzzo, L., Franceschini, A., et al. 2007, ApJS, 172, 270

Chabrier, G. 2003, PASP, 115, 763

Chapman, S. C., Blain, A., Ibata, R., et al. 2009, ApJ, 691, 560

Chapman, S. C., Bertoldi, F., Smail, I., et al. 2015, MNRAS, 449, L68

Chartab, N., Mobasher, B., Shapley, A. E., et al. 2021, ApJ, 908, 120

Chiang, Y.-K., Overzier, R., \& Gebhardt, K. 2013, ApJ, 779, 127

Chiang, Y.-K., Overzier, R., \& Gebhardt, K. 2014, ApJ, 782, L3

Chiang, Y.-K., Overzier, R. A., Gebhardt, K., et al. 2015, ApJ, 808, 37

Chiang, Y.-K., Overzier, R. A., Gebhardt, K., \& Henriques, B. 2017, ApJ, 844, L23

Ciesla, L., Charmandaris, V., Georgakakis, A., et al. 2015, A\&A, 576, A10

Civano, F., Elvis, M., Brusa, M., et al. 2012, ApJS, 201, 30

Civano, F., Marchesi, S., Comastri, A., et al. 2016, ApJ, 819, 62

Clements, D. L., Braglia, F. G., Hyde, A. K., et al. 2014, MNRAS, 439, 1193

Clements, D. L., Braglia, F., Petitpas, G., et al. 2016, MNRAS, 461, 1719

Cooke, E. A., Hatch, N. A., Muldrew, S. I., Rigby, E. E., \& Kurk, J. D. 2014, MNRAS, 440, 3262

Cooke, E. A., Hatch, N. A., Rettura, A., et al. 2015, MNRAS, 452, 2318 Cucciati, O., Zamorani, G., Lemaux, B. C., et al. 2014, A\&A, 570, A16 Cucciati, O., Lemaux, B. C., Zamorani, G., et al. 2018, A\&A, 619, A49 Cullen, F., McLure, R. J., Dunlop, J. S., et al. 2019, MNRAS, 487, 2038 Cullen, F., McLure, R. J., Dunlop, J. S., et al. 2020, MNRAS, 495, 1501 da Cunha, E., Walter, F., Smail, I. R., et al. 2015, ApJ, 806, 110 Dannerbauer, H., Kurk, J. D., De Breuck, C., et al. 2014, A\&A, 570, A55 Dannerbauer, H., Lehnert, M. D., Emonts, B., et al. 2017, A\&A, 608, A48 Darvish, B., Scoville, N. Z., Martin, C., et al. 2020, ApJ, 892, 8 Davidzon, I., Cucciati, O., Bolzonella, M., et al. 2016, A\&A, 586, A23 Davidzon, I., Ilbert, O., Laigle, C., et al. 2017, A\&A, 605, A70

de Jong, T., Klein, U., Wielebinski, R., \& Wunderlich, E. 1985, A\&A, 147, L6 Dekel, A., \& Birnboim, Y. 2006, MNRAS, 368, 2

Dekel, A., Sari, R., \& Ceverino, D. 2009, ApJ, 703, 785

Diener, C., Lilly, S. J., Ledoux, C., et al. 2015, ApJ, 802, 31

Digby-North, J. A., Nandra, K., Laird, E. S., et al. 2010, MNRAS, 407, 846
Draine, B. T., \& Li, A. 2007, ApJ, 657, 810

Eisenhardt, P. R. M., Brodwin, M., Gonzalez, A. H., et al. 2008, ApJ, 684, 905 Elbaz, D., Dickinson, M., Hwang, H. S., et al. 2011, A\&A, 533, A119 Euclid Collaboration (Adam, R., et al.) 2019, A\&A, 627, A23

Fanelli, M. N., O’Connell, R. W., \& Thuan, T. X. 1988, ApJ, 334, 665 Feltre, A., Maseda, M. V., Bacon, R., et al. 2020, A\&A, 641, A118 Finkelstein, S. L., Hill, G. J., Gebhardt, K., et al. 2011, ApJ, 729, 140 Flores-Cacho, I., Pierini, D., Soucail, G., et al. 2016, A\&A, 585, A54 Fotopoulou, S., Buchner, J., Georgantopoulos, I., et al. 2016, A\&A, 587, A142 Fouqué, P., Solanes, J. M., Sanchis, T., \& Balkowski, C. 2001, A\&A, 375, 770 Fritz, J., Franceschini, A., \& Hatziminaoglou, E. 2006, MNRAS, 366, 767 Fu, H., Yan, L., Scoville, N. Z., et al. 2010, ApJ, 722, 653 Galametz, A., Vernet, J., De Breuck, C., et al. 2010, A\&A, 522, A58 García-Vergara, C., Hodge, J., Hennawi, J. F., et al. 2020, ApJ, 904, 2 Gardner, J. P., Mather, J. C., Clampin, M., et al. 2006, in SPIE Conf. Ser., eds.

J. C. Mather, H. A. MacEwen, M. W. M. de Graauw, 6265, 62650N Garilli, B., Fumana, M., Franzetti, P., et al. 2010, PASP, 122, 827 Garilli, B., Paioro, L., Scodeggio, M., et al. 2012, PASP, 124, 1232 Georgantopoulos, I., Rovilos, E., \& Comastri, A. 2011, A\&A, 526, A46 Gómez-Guijarro, C., Riechers, D. A., Pavesi, R., et al. 2019, ApJ, 872, 117 González Delgado, R. M., Heckman, T., Leitherer, C., et al. 1998, ApJ, 505, 174 Górski, K. M., Hivon, E., Banday, A. J., et al. 2005, ApJ, 622, 759 Griffin, M. J., Abergel, A., Abreu, A., et al. 2010, A\&A, 518, L3 Guo, Q., White, S., Angulo, R. E., et al. 2013, MNRAS, 428, 1351 Harrison, C. M. 2017, Nat. Astron., 1, 0165

Hasinger, G., Capak, P., Salvato, M., et al. 2019, VizieR Online Data Catalog: $\mathrm{J} / \mathrm{ApJ} / 858 / 77$

Hatch, N. A., Overzier, R. A., Kurk, J. D., et al. 2009, MNRAS, 395, 114 Hatch, N. A., Kurk, J. D., Pentericci, L., et al. 2011, MNRAS, 415, 2993 Hayashi, M., Kodama, T., Tanaka, I., et al. 2016, ApJ, 826, L28

Heckman, T. M., Robert, C., Leitherer, C., Garnett, D. R., \& van der Rydt, F. 1998, ApJ, 503, 646

Henriques, B. M. B., White, S. D. M., Thomas, P. A., et al. 2015, MNRAS, 451, 2663

Herranz, D. González-Nuevo, J., Clements, D. L., et al. 2013, A\&A, 549, A31 Hill, J. M., Green, R. F., Ashby, D. S., et al. 2012, Proc. SPIE, 8444, 84441A Horne, K. 1986, PASP, 98, 609

Hung, C.-L., Casey, C. M., Chiang, Y.-K., et al. 2016, ApJ, 826, 130

Ichikawa, T., Suzuki, R., \& Tokoku, C. 2006, in SPIE Conf. Ser., eds. I. S. McLean, \& M. Iye, 6269, 626916

Inoue, A. K., Yamanaka, S., Ouchi, M., et al. 2020, PASJ, 72, 101

Ivison, R. J., Swinbank, A. M., Smail, I., et al. 2013, ApJ, 772, 137

Jian, H.-Y., Lin, L., \& Chiueh, T. 2012, ApJ, 754, 26

Johnson, S. P., Wilson, G. W., Wang, Q. D., et al. 2013, MNRAS, 431, 662

Kato, Y., Matsuda, Y., Smail, I., et al. 2016, MNRAS, 460, 3861

Kennicutt, R. C., Jr. 1998, ARA\&A, 36, 189

Kereš, D., Katz, N., Weinberg, D. H., \& Davé, R. 2005, MNRAS, 363, 2

Kereš, D., Katz, N., Fardal, M., Davé, R., \& Weinberg, D. H. 2009, MNRAS, 395,160

Kewley, L. J., Nicholls, D. C., Sutherland, R., et al. 2019, ApJ, 880, 16

Khachikian, E. Y., \& Weedman, D. W. 1974, ApJ, 192, 581

Kinney, A. L., Bohlin, R. C., Calzetti, D., Panagia, N., \& Wyse, R. F. G. 1993, ApJS, 86, 5

Kneissl, R., Polletta, M., Martinache, C., et al. 2019, A\&A, 625, A96

Koyama, Y., Kodama, T., Tadaki, K.-I., et al. 2013, MNRAS, 428, 1551

Koyama, Y., Polletta, M. D. C., Tanaka, I., et al. 2021, MNRAS, 503, L1

Kravtsov, A. V., \& Borgani, S. 2012, ARA\&A, 50, 353

Kriek, M., Shapley, A. E., Reddy, N. A., et al. 2015, ApJS, 218, 15

Krishnan, C., Hatch, N. A., Almaini, O., et al. 2017, MNRAS, 470, 2170

Kuiper, E., Venemans, B. P., Hatch, N. A., Miley, G. K., \& Röttgering, H. J. A 2012, MNRAS, 425, 801

Lacaille, K. M., Chapman, S. C., Smail, I., et al. 2019, MNRAS, 488, 1790

Lahav, O., Lilje, P. B., Primack, J. R., \& Rees, M. J. 1991, MNRAS, 251, 128

Laigle, C., McCracken, H. J., Ilbert, O., et al. 2016, ApJS, 224, 24

Laird, E. S., Nandra, K., Pope, A., \& Scott, D. 2010, MNRAS, 401, 2763

Lapiner, S., Dekel, A., \& Dubois, Y. 2021, MNRAS, 505, 172

Laureijs, R., Amiaux, J., Arduini, S., et al. 2011, Euclid Definition Study Report (Red Book), ESA/SRE(2011), 12

Le Fèvre, O., Cassata, P., Cucciati, O., et al. 2013, A\&A, 559, A14

Lee, M. M., Tanaka, I., Kawabe, R., et al. 2017, ApJ, 842, 55

Lehmer, B. D., Alexander, D. M., Geach, J. E., et al. 2009, ApJ, 691, 687

Lehmer, B. D., Lucy, A. B., Alexander, D. M., et al. 2013, ApJ, 765, 87

Leitherer, C., Schaerer, D., Goldader, J. D., et al. 1999, ApJS, 123, 3

Leitherer, C., Tremonti, C. A., Heckman, T. M., \& Calzetti, D. 2011, AJ, 141, 37

Lemaux, B. C., Cucciati, O., Tasca, L. A. M., et al. 2014, A\&A, 572, A41

Lemaux, B. C., Le Fèvre, O., Cucciati, O., et al. 2018, A\&A, 615, A77

Lemaux, B. C., Cucciati, O., Le Fèvre, O., et al. 2020, A\&A, submitted [arXiv:2009.03324] 
Lilly, S. J., Le Fèvre, O., Renzini, A., et al. 2007, ApJS, 172, 70

Lilly, S. J., Le Brun, V., Maier, C., et al. 2009, ApJS, 184, 218

Lim, S., Scott, D., Babul, A., et al. 2021, MNRAS, 501, 1803

Long, A. S., Cooray, A., Ma, J., et al. 2020, ApJ, 898, 133

Lusso, E., Comastri, A., Simmons, B. D., et al. 2012, MNRAS, 425, 623

Macuga, M., Martini, P., Miller, E. D., et al. 2019, ApJ, 874, 54

Madau, P., \& Dickinson, M. 2014, ARA\&A, 52, 415

Magnelli, B., Ivison, R. J., Lutz, D., et al. 2015, A\&A, 573, A45

Mantz, A. B., Allen, S. W., Morris, R. G., et al. 2017, MNRAS, 472, 2877

Martinache, C., Rettura, A., Dole, H., et al. 2018, A\&A, 620, A198

Matsuda, Y., Yamada, T., Hayashino, T., et al. 2005, ApJ, 634, L125

McCracken, H. J., Milvang-Jensen, B., Dunlop, J., et al. 2012, A\&A, 544, A156

Miller, T. B., Hayward, C. C., Chapman, S. C., \& Behroozi, P. S. 2015, MNRAS 452,878

Miller, T. B., Chapman, S. C., Aravena, M., et al. 2018, Nature, 556, 469

Mitsuhashi, I., Matsuda, Y., Smail, I., et al. 2021, ApJ, 907, 122

Mo, H. J., \& White, S. D. M. 1996, MNRAS, 282, 347

Muldrew, S. I., Hatch, N. A., \& Cooke, E. A. 2015, MNRAS, 452, 2528

Nantais, J. B., Muzzin, A., van der Burg, R. F. J., et al. 2017, MNRAS, 465, L104

Narayanan, D., Turk, M., Feldmann, R., et al. 2015, Nature, 525, 496

Negrello, M., González-Nuevo, J., Magliocchetti, M., et al. 2005, MNRAS, 358 869

Negrello, M., Gonzalez-Nuevo, J., De Zotti, G., et al. 2017, MNRAS, 470, 2253

Nguyen, H. T., Schulz, B., Levenson, L., et al. 2010, A\&A, 518, L5

Noirot, G., Vernet, J., De Breuck, C., et al. 2016, ApJ, 830, 90

Noirot, G., Stern, D., Mei, S., et al. 2018, ApJ, 859, 38

Noll, S., Burgarella, D., Giovannoli, E., et al. 2009, A\&A, 507, 1793

Oliver, S. J., Bock, J., Altieri, B., et al. 2012, MNRAS, 424, 1614

Oteo, I., Ivison, R. J., Dunne, L., et al. 2018, ApJ, 856, 72

Overzier, R. A. 2016, A\&A Rv, 24, 14

Overzier, R. A., Guo, Q., Kauffmann, G., et al. 2009, MNRAS, 394, 577

Pavesi, R., Riechers, D. A., Sharon, C. E., et al. 2018, ApJ, 861, 43

Pentericci, L., Kurk, J. D., Röttgering, H. J. A., et al. 2000, A\&A, 361, L25

Percival, W. J. 2005, A\&A, 443, 819

Pilbratt, G. L., Riedinger, J. R., Passvogel, T., et al. 2010, A\&A, 518, L1

Planck Collaboration I. 2011, A\&A, 536, A1

Planck Collaboration VI. 2020, A\&A, 641, A6

Planck Collaboration Int. XXVII. 2015, A\&A, 582, A30

Planck Collaboration Int. XXXIX. 2016, A\&A, 596, A100

Polletta, M., Dole, H., Martinache, C., et al. 2021, A\&A submitted

Ranalli, P., Koulouridis, E., Georgantopoulos, I., et al. 2016, A\&A, 590, A80

Rieke, G. H., Alonso-Herrero, A., Weiner, B. J., et al. 2009, ApJ, 692, 556

Rix, S. A., Pettini, M., Leitherer, C., et al. 2004, ApJ, 615, 98

Robitaille, T., \& Bressert, E. 2012, APLpy: Astronomical Plotting Library in Python

Rodighiero, G., Daddi, E., Baronchelli, I., et al. 2011, ApJ, 739, L40

Roseboom, I. G., Oliver, S. J., Kunz, M., et al. 2010, MNRAS, 409, 48

Rosito, M. S., Pedrosa, S. E., Tissera, P. B., et al. 2021, A\&A, 652, A44

Salpeter, E. E. 1955, ApJ, 121, 161

Sanders, D. B., Soifer, B. T., Elias, J. H., Neugebauer, G., \& Matthews, K. 1988, ApJ, 328, L35

Sanders, D. B., Salvato, M., Aussel, H., et al. 2007, ApJS, 172, 86

Sargent, M. T., Daddi, E., Béthermin, M., et al. 2014, ApJ, 793, 19

Sattari, Z., Mobasher, B., Chartab, N., et al. 2021, ApJ, 910, 57

Schlafly, E. F., \& Finkbeiner, D. P. 2011, ApJ, 737, 103

Scodeggio, M., Franzetti, P., Garilli, B., et al. 2005, PASP, 117, 1284

Scoville, N., Aussel, H., Brusa, M., et al. 2007, ApJS, 172, 1

Seifert, W., Appenzeller, I., Baumeister, H., et al. 2003, in Instrument Design and Performance for Optical/Infrared Ground-based Telescopes, eds. M. Iye \& A. F. M. Moorwood, Proc. SPIE, 4841, 962

Seifert, W., Ageorges, N., Lehmitz, M., et al. 2010, Proc. SPIE, 7735, 77357W

Shahidi, A., Mobasher, B., Nayyeri, H., et al. 2020, ApJ, 897, 44

Shapley, A. E., Steidel, C. C., Pettini, M., \& Adelberger, K. L. 2003, ApJ, 588 , 65

Shimakawa, R., Kodama, T., Tadaki, K. I., et al. 2014, MNRAS, 441, L1

Shimakawa, R., Kodama, T., Tadaki, K.-I., et al. 2015, MNRAS, 448, 666

Shimakawa, R., Koyama, Y., Röttgering, H. J. A., et al. 2018, MNRAS, 481, 5630
Silverman, J. D., Green, P. J., Barkhouse, W. A., et al. 2008, ApJ, 679, 118 Silverman, J. D., Kashino, D., Sanders, D., et al. 2015, ApJS, 220, 12

Smith, C. M. A., Gear, W. K., Smith, M. W. L., Papageorgiou, A., \& Eales, S. A. 2019, MNRAS, 486, 4304

Smolčić, V., Novak, M., Bondi, M., et al. 2017, A\&A, 602, A1

Sommariva, V., Mannucci, F., Cresci, G., et al. 2012, A\&A, 539, A136

Sopp, H. M., \& Alexander, P. 1991, MNRAS, 251, 14P

Speagle, J. S., Steinhardt, C. L., Capak, P. L., \& Silverman, J. D. 2014, ApJS, 214,15

Spitler, L. R., Labbé, I., Glazebrook, K., et al. 2012, ApJ, 748, L21

Steidel, C. C., Adelberger, K. L., Dickinson, M., et al. 1998, ApJ, 492, 428

Steidel, C. C., Adelberger, K. L., Shapley, A. E., et al. 2005, ApJ, 626, 44

Strazzullo, V., Daddi, E., Gobat, R., et al. 2016, ApJ, 833, L20

Strazzullo, V., Coogan, R. T., Daddi, E., et al. 2018, ApJ, 862, 64

Strazzullo, V., Pannella, M., Mohr, J. J., et al. 2019, A\&A, 622, A117

Tadaki, K.-I., Kodama, T., Hayashi, M., et al. 2019, PASJ, 71, 40

Tanaka, I., Breuck, C. D., Kurk, J. D., et al. 2011, PASJ, 63, 415

Tasca, L. A. M., Kneib, J. P., Iovino, A., et al. 2009, A\&A, 503, 379

Taylor, M. B. 2005, in Astronomical Data Analysis Software and Systems XIV, eds. P. Shopbell, M. Britton, \& R. Ebert, ASP Conf. Ser., 347, 29

Trump, J. R., Impey, C. D., McCarthy, P. J., et al. 2007, ApJS, 172, 383

Trump, J. R., Impey, C. D., Elvis, M., et al. 2009, ApJ, 696, 1195

Umehata, H., Fumagalli, M., Smail, I., et al. 2019, Science, 366, 97

Umehata, H., Tamura, Y., Kohno, K., et al. 2015, ApJ, 815, L8

Umehata, H., Tamura, Y., Kohno, K., et al. 2017, ApJ, 835, 98

Valentino, F., Daddi, E., Strazzullo, V., et al. 2015, ApJ, 801, 132

Valentino, F., Daddi, E., Finoguenov, A., et al. 2016, ApJ, 829, 53

Venemans, B. P., Röttgering, H. J. A., Miley, G. K., et al. 2007, A\&A, 461, 823

Ventou, E., Contini, T., Bouché, N., et al. 2017, A\&A, 608, A9

Vidal-García, A., Charlot, S., Bruzual, G., \& Hubeny, I. 2017, MNRAS, 470, 3532

Wagner, C. R., Courteau, S., Brodwin, M., et al. 2017, ApJ, 834, 53

Wang, L., Viero, M., Clarke, C., et al. 2014, MNRAS, 444, 2870

Wang, T., Elbaz, D., Daddi, E., et al. 2016, ApJ, 828, 56

Wang, T., Elbaz, D., Daddi, E., et al. 2018, ApJ, 867, L29

Wilkins, S. M., Bouwens, R. J., Oesch, P. A., et al. 2016, MNRAS, 455, 659

Williams, R. J., Quadri, R. F., Franx, M., van Dokkum, P., \& Labbé, I. 2009, ApJ, 691, 1879

Woo, J.-H., \& Urry, C. M. 2002, ApJ, 579, 530

Wright, E. L. 2006, PASP, 118, 1711

Wunderlich, E., Klein, U., \& Wielebinski, R. 1987, A\&AS, 69, 487

Yuan, T., Nanayakkara, T., Kacprzak, G. G., et al. 2014, ApJ, 795, L20

Zetterlund, E., Levesque, E. M., Leitherer, C., \& Danforth, C. W. 2015, ApJ, 805,151

1 INAF - Istituto di Astrofisica Spaziale e Fisica cosmica (IASF) Milano, Via A. Corti 12, 20133 Milan, Italy e-mail: maria.polletta@inaf.it

2 IRAP, Université de Toulouse, CNRS, CNES, UPS, Toulouse, France

3 Université Paris-Saclay, Institut d'Astrophysique Spatiale, CNRS, Bât. 121, 91400 Orsay, France

4 Université Lyon 1, ENS de Lyon, CNRS UMR5574, Centre de Recherche Astrophysique de Lyon, 69230 Saint-Genis-Laval, France

5 Subaru Telescope, National Astronomical Observatory of Japan, 650 North A'ohoku Place, Hilo, HI 96720, USA

6 Graduate University for Advanced Studies (SOKENDAI), Osawa 221-1, Mitaka, Tokyo 181-8588, Japan

7 Aix Marseille University, CNRS, CNES, LAM, Marseille, France

8 Department of Astronomy/Steward Observatory, 933 North Cherry Avenue, University of Arizona, Tucson, AZ 85721, USA

9 INAF - Osservatorio di Astrofisica e Scienza delle Spazio di Bologna, OAS, Via Gobetti 93/3, 40129 Bologna, Italy 


\section{Appendix A: LBT spectra}

The complete list of the LBT spectroscopic targets, with coordinates, estimated redshifts, and associated quality flags, K-band magnitudes, and IRAC colors from the L16 catalog, is shown in Table A.1. In Fig. A.1 we show the LBT/LUCI spectra of the 15 LUCI selected targets. The six LBT targets that had been previously observed by the Cosmos team and the available zCosmosDeep (Lilly et al. 2009) redshifts are listed in Table A.1, and relative spectra are shown in Fig. A.2.

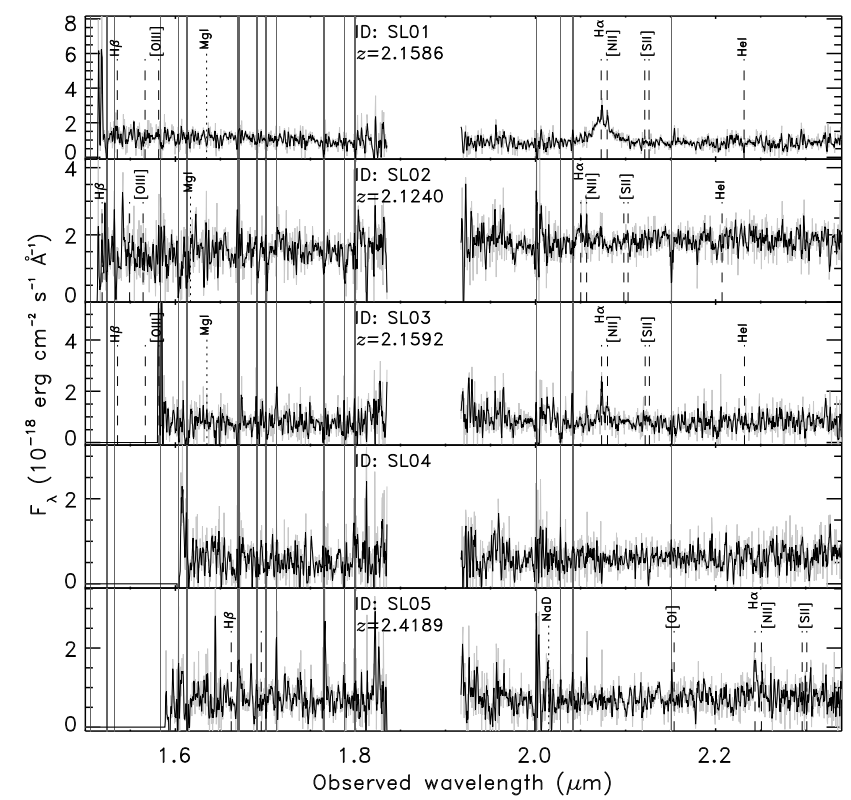

Fig. A.1. LBT/LUCI spectra (gray line, black lines are rebinned spectra) of the 15 observed targets listed in Table A.1. Identifiers are annotated and spectroscopic redshifts are shown when they could be reliably measured. In such cases, the main emission and absorption lines are shown with vertical dashed, and dotted lines, respectively. Spectral regions contaminated by bright sky lines are marked with dark gray vertical bands. Spectral features expected at the optical redshift provided by zCosmos-deep spectrum, when available (see Table A.1), are shown as red vertical dashed lines and annotated.
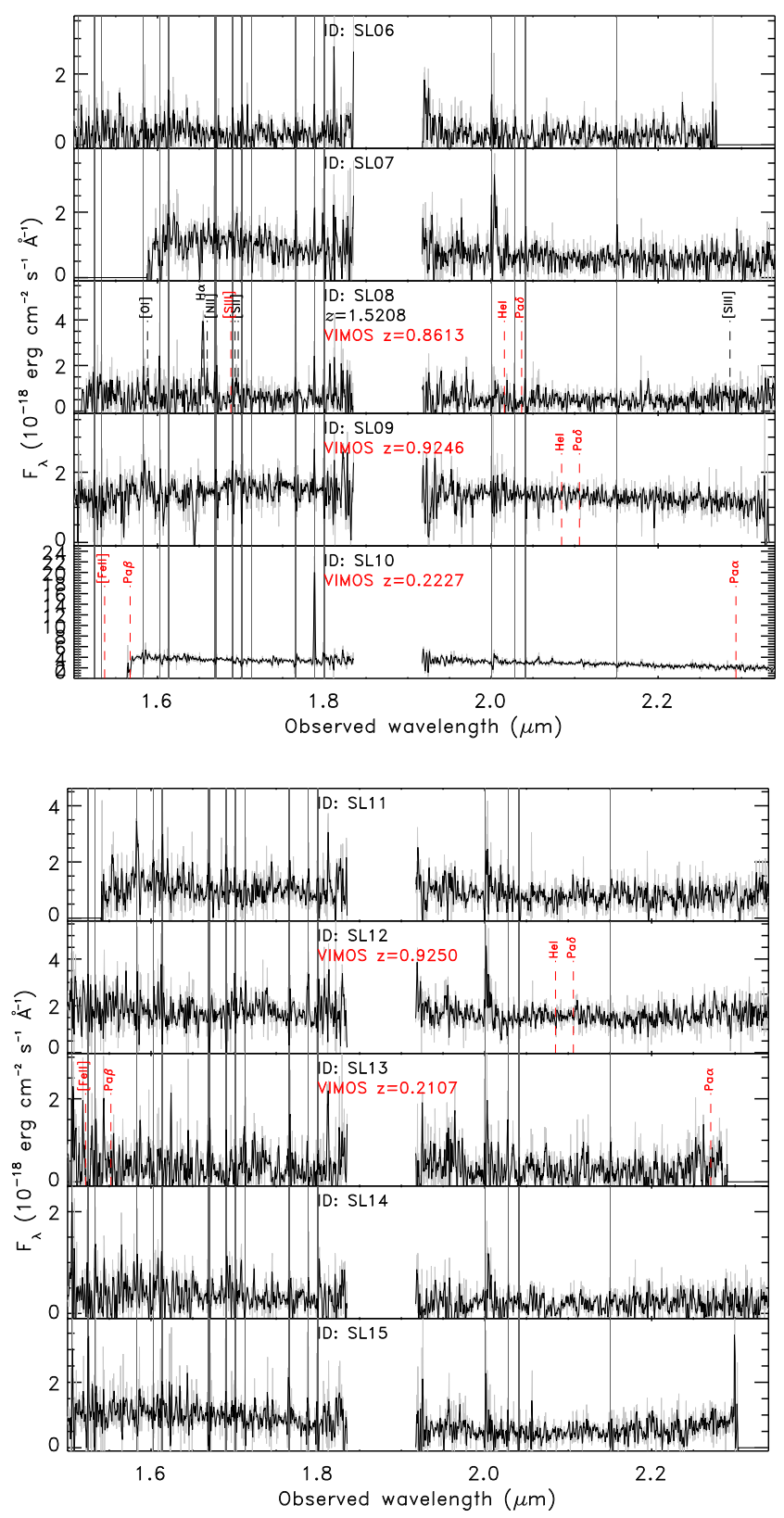

Fig. A.1. continued. 
Table A.1. LUCI targets' main properties.

\begin{tabular}{|c|c|c|c|c|c|c|c|c|}
\hline $\begin{array}{r}\text { LUCI } \\
\text { ID }\end{array}$ & $\begin{array}{c}\text { NUMBER } \\
\text { (L16) }\end{array}$ & $\begin{array}{c}\alpha(\mathrm{L} 16) \\
(\mathrm{deg})\end{array}$ & $\begin{array}{c}\delta(\mathrm{L} 16) \\
(\mathrm{deg})\end{array}$ & $\begin{array}{c}z_{\text {LUCI }} \\
\left(z_{\text {VIMOS }}\right)^{a}\end{array}$ & $\begin{array}{c}z \\
\text { flag }^{b}\end{array}$ & $\begin{array}{c}\mathrm{K}_{s} \\
(\mathrm{AB})\end{array}$ & $\mathrm{S}_{4.5 \mu \mathrm{m}} / \mathrm{S}_{3.6 \mu \mathrm{m}}$ & $\begin{array}{l}\text { Herschel } \\
\text { association }\end{array}$ \\
\hline SL01 & 679779 & 150.469450 & 2.33129 & $2.1586^{c}$ & 3 & $21.788 \pm 0.004$ & $1.083 \pm 0.014$ & 7814 \\
\hline SL02 & 676097 & 150.472301 & 2.324637 & 2.1240 & 3 & $20.099 \pm 0.001$ & $1.398 \pm 0.008$ & 2889 \\
\hline SL03 & 669706 & 150.466518 & 2.315597 & 2.1592 & 3 & $20.802 \pm 0.002$ & $1.192 \pm 0.008$ & 9741 \\
\hline SL04 & 656918 & 150.472125 & 2.296723 & $\ldots$ & $\ldots$ & $21.341 \pm 0.003$ & $1.327 \pm 0.005$ & 4103 \\
\hline SL05 & 643336 & 150.483696 & 2.276123 & 2.4189 & 2 & $21.188 \pm 0.002$ & $1.239 \pm 0.009$ & 1572 \\
\hline SL06 & 657340 & 150.506844 & 2.297430 & $\ldots$ & $\ldots$ & $21.994 \pm 0.006$ & $1.240 \pm 0.011$ & $\ldots$ \\
\hline SL07 & 665034 & 150.468504 & 2.309183 & $\ldots$ & $\ldots$ & $21.615 \pm 0.004$ & $0.741 \pm 0.047$ & \\
\hline SL08 & 664921 & 150.480758 & 2.309062 & $1.5210(0.8613)$ & $4(1)$ & $21.839 \pm 0.005$ & $1.148 \pm 0.010$ & $\ldots$ \\
\hline SL09 & 673976 & 150.486251 & 2.320757 & $(0.9246)$ & (3) & $19.816 \pm 0.001$ & $0.712 \pm 0.009$ & $\ldots$ \\
\hline SL10 & 647891 & 150.485378 & 2.280741 & $(0.2227)$ & (4) & $19.711 \pm 0.001$ & $0.797 \pm 0.016$ & $\ldots$ \\
\hline SL11 & 657494 & 150.481342 & 2.297394 & $\ldots$ & $\ldots$ & $21.226 \pm 0.003$ & $0.713 \pm 0.025$ & $\ldots$ \\
\hline SL12 & 658044 & 150.489417 & 2.297995 & $(0.9250)$ & (3) & $20.360 \pm 0.001$ & $0.727 \pm 0.012$ & $\ldots$ \\
\hline SL13 & 659137 & 150.502422 & 2.300324 & $(0.2107)$ & $\ldots$ & $21.689 \pm 0.004$ & $0.884 \pm 0.009$ & $\ldots$ \\
\hline SL14 & 674760 & 150.475890 & 2.323199 & $\ldots$ & $\ldots$ & $21.482 \pm 0.003$ & $0.755 \pm 0.036$ & $\ldots$ \\
\hline SL15 & 647673 & 150.508934 & 2.281465 & $\ldots$ & $\ldots$ & $20.529 \pm 0.001$ & $0.761 \pm 0.022$ & $\ldots$ \\
\hline
\end{tabular}

Notes. ${ }^{a}$ VISMOS spectroscopic redshifts are from the zCosmos spectroscopic survey (Lilly et al. 2007, 2009). ${ }^{b}$ The redshift flag corresponds to the probability of the redshift estimate of being correct and can be equal to 1 (50-75\% probability), 2 (75-90\% probability), or to 3 , and 4 ( $>90 \%$ probability; Le Fèvre et al. 2013). ${ }^{c}$ The LUCI spectrum shows a broad $\mathrm{H} \alpha$ line, implying that this source is an AGN.

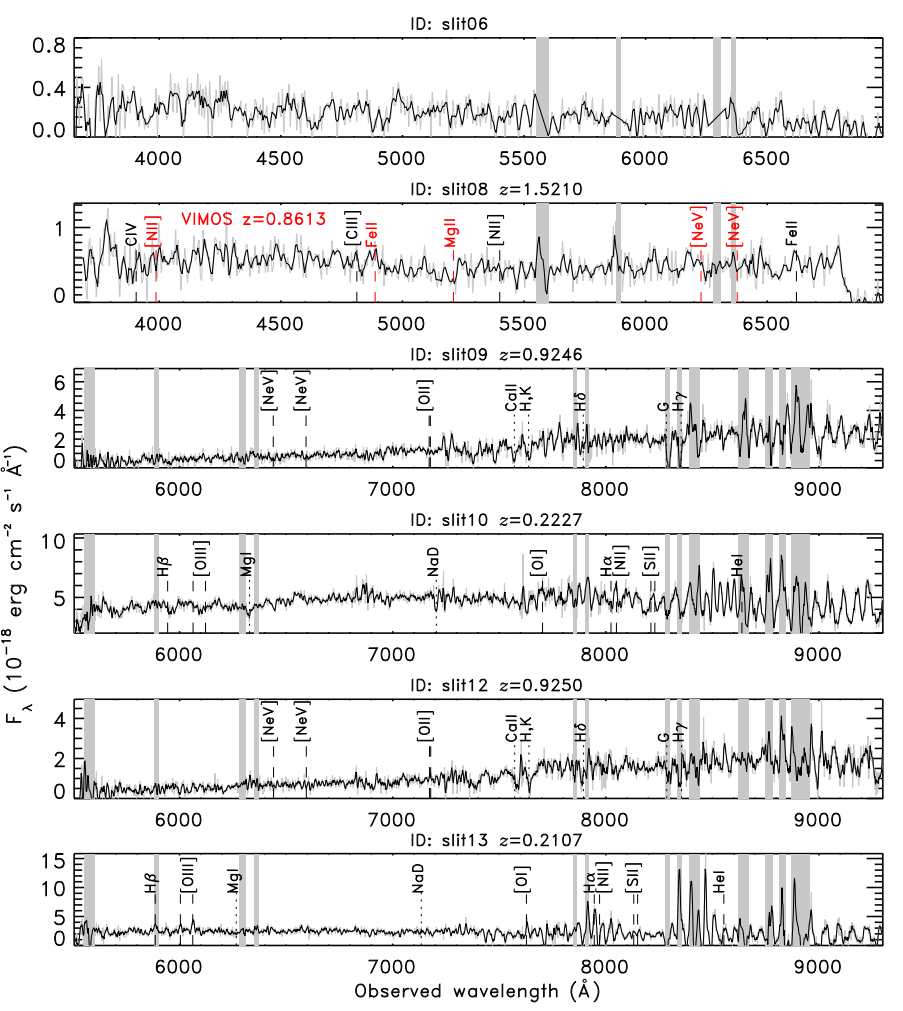

Fig. A.2. zCosmos-Deep VIMOS spectra of the sources observed with LBT/LUCI (gray line, in black after rebinning). Identifiers and spectroscopic redshifts are annotated at the top of each panel. The main emission and absorption lines are shown with vertical dashed, or dotted lines, respectively. Spectral regions contaminated by bright sky lines are marked with gray vertical bands. In case of ID slit08, we show the spectral features expected at the LBT/LUCI redshift in black, and those at the VIMOS optical redshift in red. The LUCI redshift is more reliable than the VIMOS one in this case. 


\section{Appendix B: VIMOS spectra}

In Fig. B.1, we show the zCosmos-Deep/VIMOS spectra of the sources at $2.15 \leq z_{\text {spec }}<2.164$, in Fig. B.2, those $2.19<z_{\text {spec }}<2.20$, and in Fig. B.3 those at $2.164<z_{\text {spec }}<2.19$.
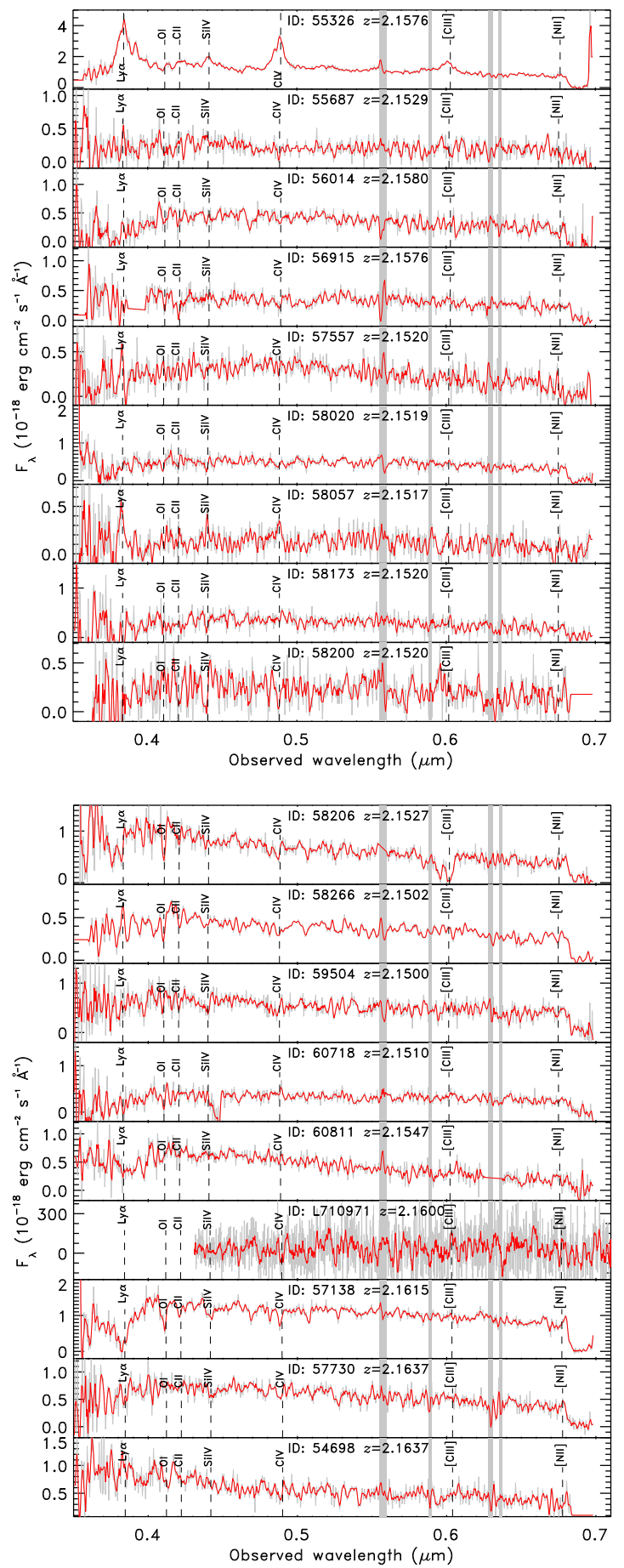

Fig. B.1. VIMOS (DEIMOS for L710971) spectra (gray line) of 18 member candidates with $z=2.15-2.164$ in the $10^{\prime} \times 12^{\prime}$ region centered at $\alpha=150.465^{\circ}$, and $\delta=2.31^{\circ}$ where $\mathrm{G} 237$ is located. The red lines are smoothed spectra. The vertical dashed lines represent the location of the main spectral lines as annotated. Source IDs and spectroscopic redshifts are annotated in each panel. Regions contaminated by bright sky lines are highlighted with vertical gray bars.

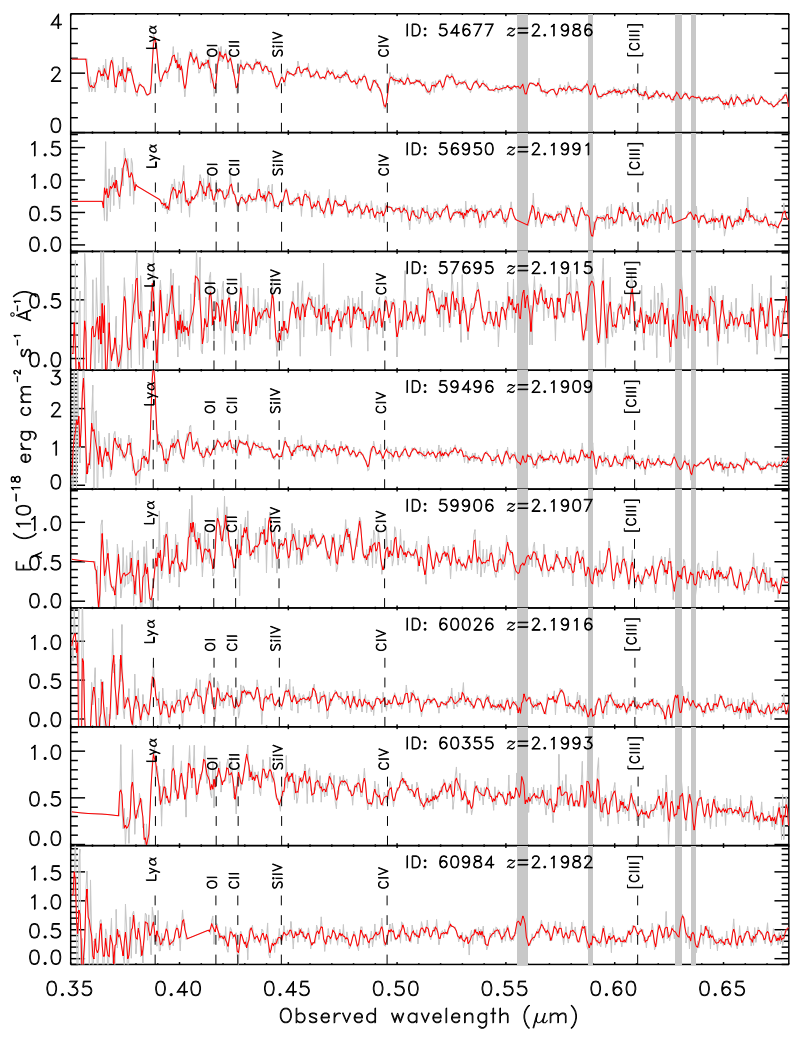

Fig. B.2. VIMOS spectra (gray line) of eight member candidates with $z=2.19-2.20$ in the $10^{\prime} \times 12^{\prime}$ region centered at $\alpha=150.465^{\circ}$, and $\delta=2.31^{\circ}$ where $\mathrm{G} 237$ is located. The red lines are smoothed spectra. The vertical dashed lines represent the location of the main spectral lines as annotated. Source IDs and spectroscopic redshifts are annotated in each panel. Regions contaminated by bright sky lines are highlighted with vertical gray bars.

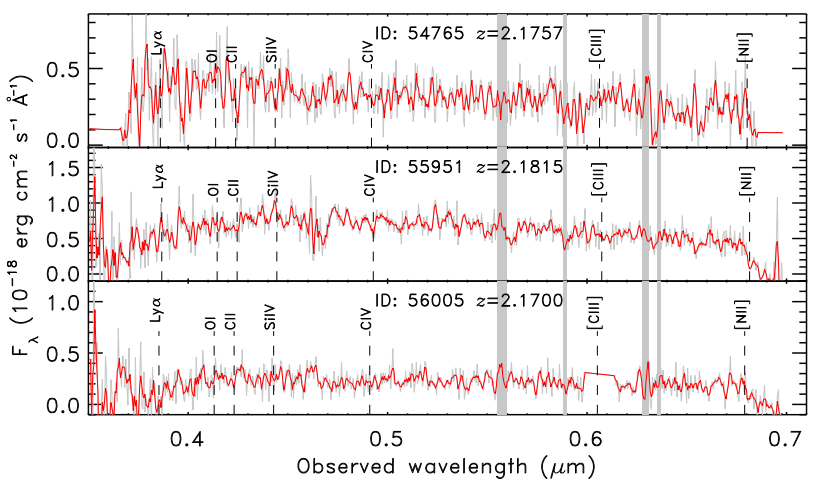

Fig. B.3. VIMOS spectra (gray line) of three member candidates with $z=2.164-2.19$ in the $10^{\prime} \times 12^{\prime}$ region centered at $\alpha=150.465^{\circ}$, and $\delta=2.31^{\circ}$ where $\mathrm{G} 237$ is located. The red lines are smoothed spectra. The vertical dashed lines represent the location of the main spectral lines as annotated. Source IDs and spectroscopic redshifts are annotated in each panel. Regions contaminated by bright sky lines are highlighted with vertical gray bars. 


\section{Appendix C: Stacked spectrum assessment}

In Fig. C.1, we show the co-added spectra obtained with all 26 non AGN sources at $z=2.15-2.20$ (full stack in the top panel), with the 16 galaxies with $z$ flg $\geq 2$ (clean stack in the middle panel), and with the 12 galaxies at $z=2.15-2.16$ (bottom panel).

All three co-added spectra show numerous absorption features and are consistent within $1 \sigma$, providing evidence that most redshift estimates with low zflg are correct. The median $\mathrm{S} / \mathrm{N}$ of the full stack is 14 , that of the clean stack is 12 , and of the $z=2.15-2.16$ stack is 8.4. In Sect. 5.3, we carry out the spectral analysis of the "full stack" spectrum because has a higher $\mathrm{S} / \mathrm{N}$ and it is more complete and representative of the member population.

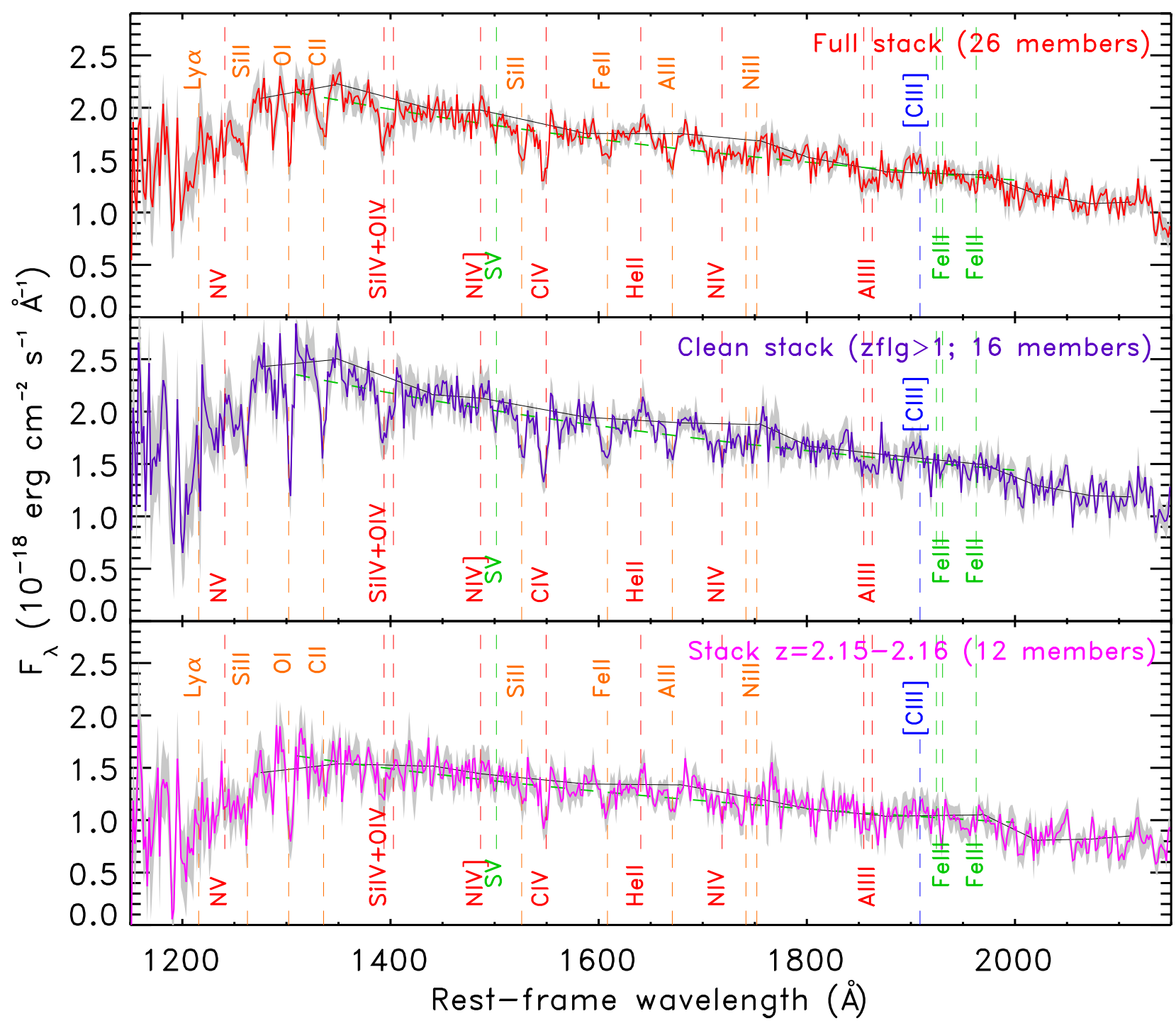

Fig. C.1. Stacked rest-frame VIMOS spectra of 26 member candidates with $z=2.15-2.20$ (red solid line in top panel), of 16 members with $z f g \geq 2$ (purple solid line in middle panel), and of 12 members with $z=2.15-2.16$ (magenta solid line in bottom panel). In all panels, the gray filled region represents the $1 \sigma$ uncertainty, the dashed green line is a linear fit obtained to estimate the UV spectral slope $\beta$, where $\mathrm{F}_{\lambda} \propto \lambda^{\beta}$, and the solid black curve represents the pseudo-continuum obtained by linearly interpolating the average flux density in the "features-free" windows defined in Table 3 in Rix et al. (2004). The vertical dashed lines represent the location of the main spectral features as annotated (nebular: blue, ISM: orange, stellar photosphere: green, stellar winds: red). 


\section{Appendix D: Morphological analysis}

Postage stamps $\left(6^{\prime \prime} \times 6^{\prime \prime}\right)$ in the HST/ACS F814W-band and in the UltraVISTA/YJH bands of all 31 spectroscopic members are shown in Fig. D.1, D.2, and D.3. The identifier (ID) and the morphological classification are reported in the figure captions.

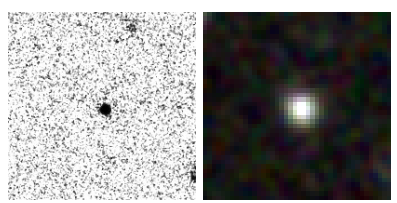

(a)

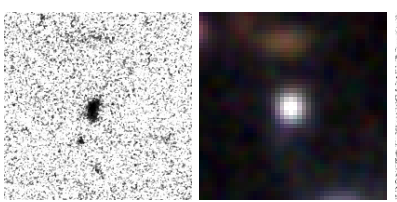

(e)

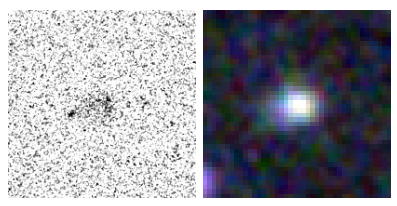

(i)

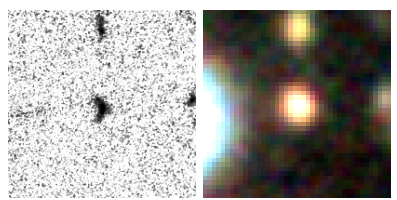

(m)

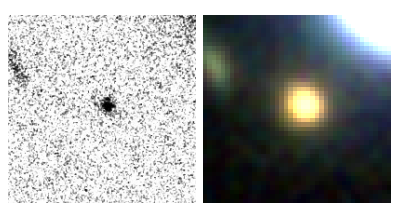

(q)

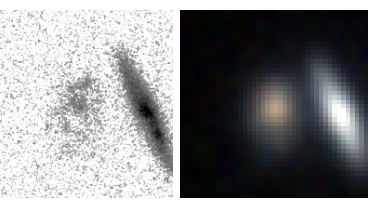

(b)

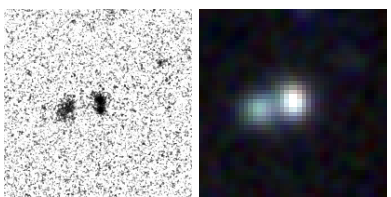

(f)

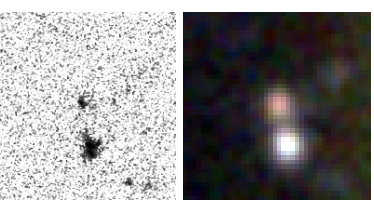

(j)

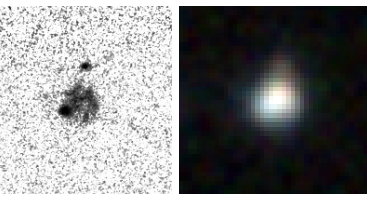

(n)

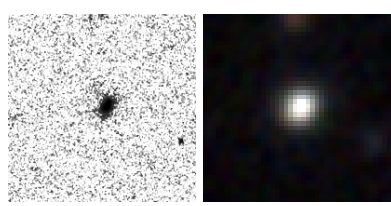

(r)

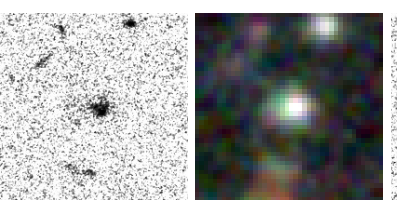

(c)

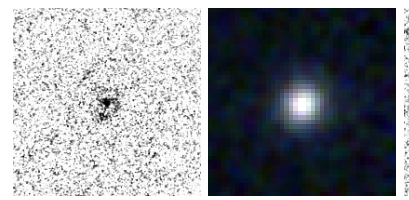

(g)

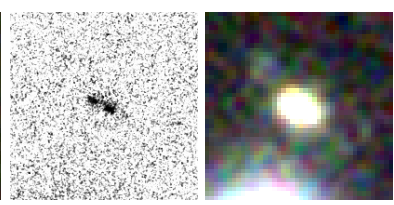

$(\mathrm{k})$

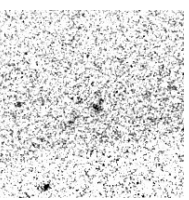

(o)

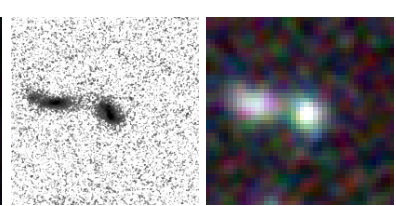

(s)

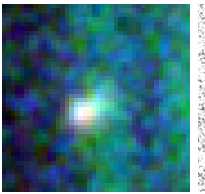

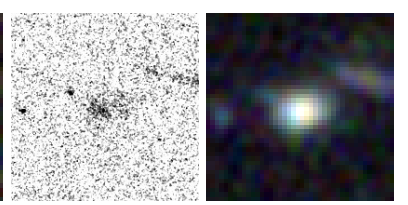

(d)

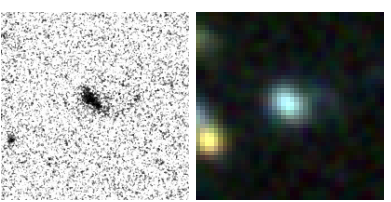

(h)

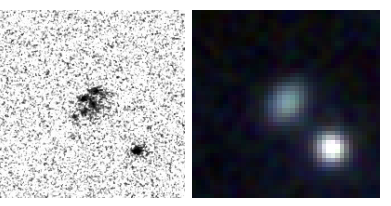

(1)

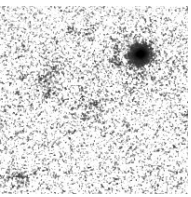

(p)
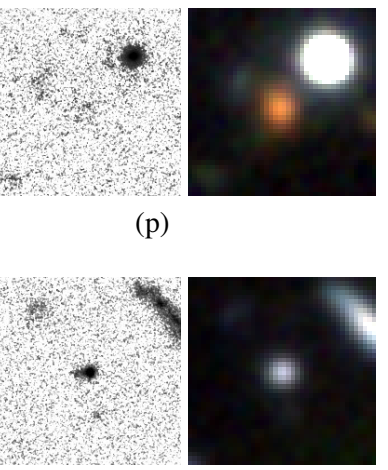

(t)

Fig. D.1. $6^{\prime \prime} \times 6^{\prime \prime}$ ACS I-band and UltraVISTA YJH cutouts of the sources at $2.15 \leq z<2.164$. Source ID and morphological classification are the following: (a) 55326: ET, (b) 55687: Disk, (c) 56014: Irr, (d) 56915: Disk, (e) 57557: Disk, (f) 58020: Irr, (g) 58057: Disk, (h) 58173: Irr, (i) 58200: Too faint, (j) 58206: Disk, (k) 58266: Irr, (1) 59504: Disk, (m) 60718: Disk, (n) 60811: Disk, (o) L710971: Too faint, (p) SL01: Too faint, (q) SL03: Disk, (r) 57138: Disk, (s) 54698: Irr, (t) 57730: Disk.

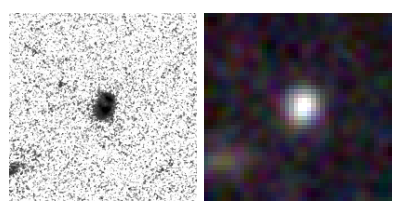

(a)

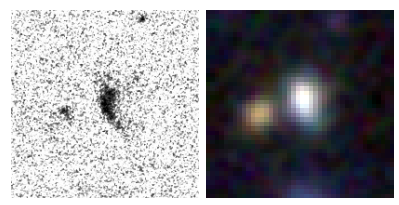

(e)

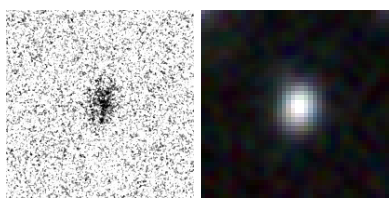

(b)

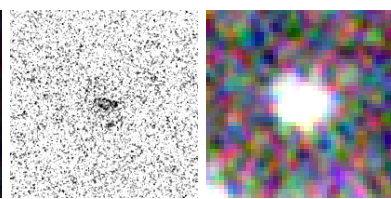

(f)

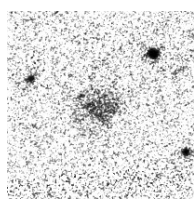

(c)

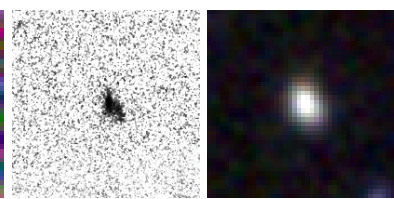

(g)

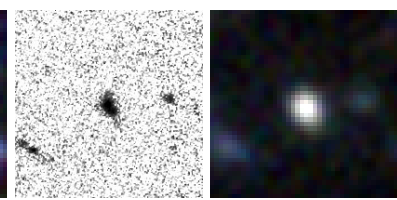

(d)

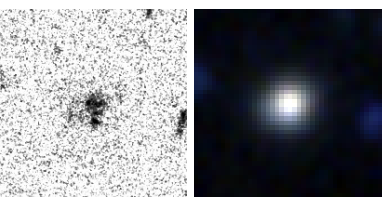

(h)

Fig. D.2. 6" $\times 6$ 6" ACS I-band and UltraVISTA YJH cutouts of the sources at $z>2.19$. Source ID and morphological classification are the following: (a) 54677: Irr, (b) 56950: Irr, (c) 57695: Irr, (d) 59496: Disk, (e) 59906: Irr, (f) 60026: Disk, (g) 60355: Irr, (h) 60984: Irr. 


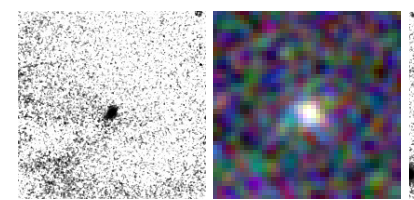

(a)

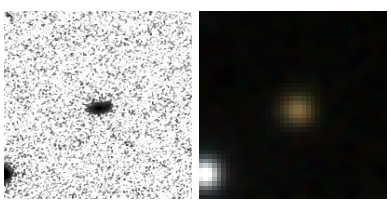

(b)

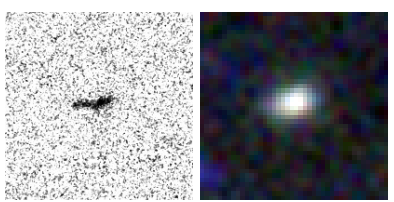

(c)

Fig. D.3. $6^{\prime \prime} \times 6^{\prime \prime}$ ACS I-band and UltraVISTA YJH cutouts of the sources at $2.164<z<2.19$. Source ID and morphological classification are the following: (a) 54765: Disk, (b) 55951: Disk, (c) 56005: Disk.

\section{Appendix E: Spectral energy distributions}

In Fig. E.1, we show the SEDs of the 31 spectroscopic members and the best fit models obtained with CIGALE (see Sect. 5.4).

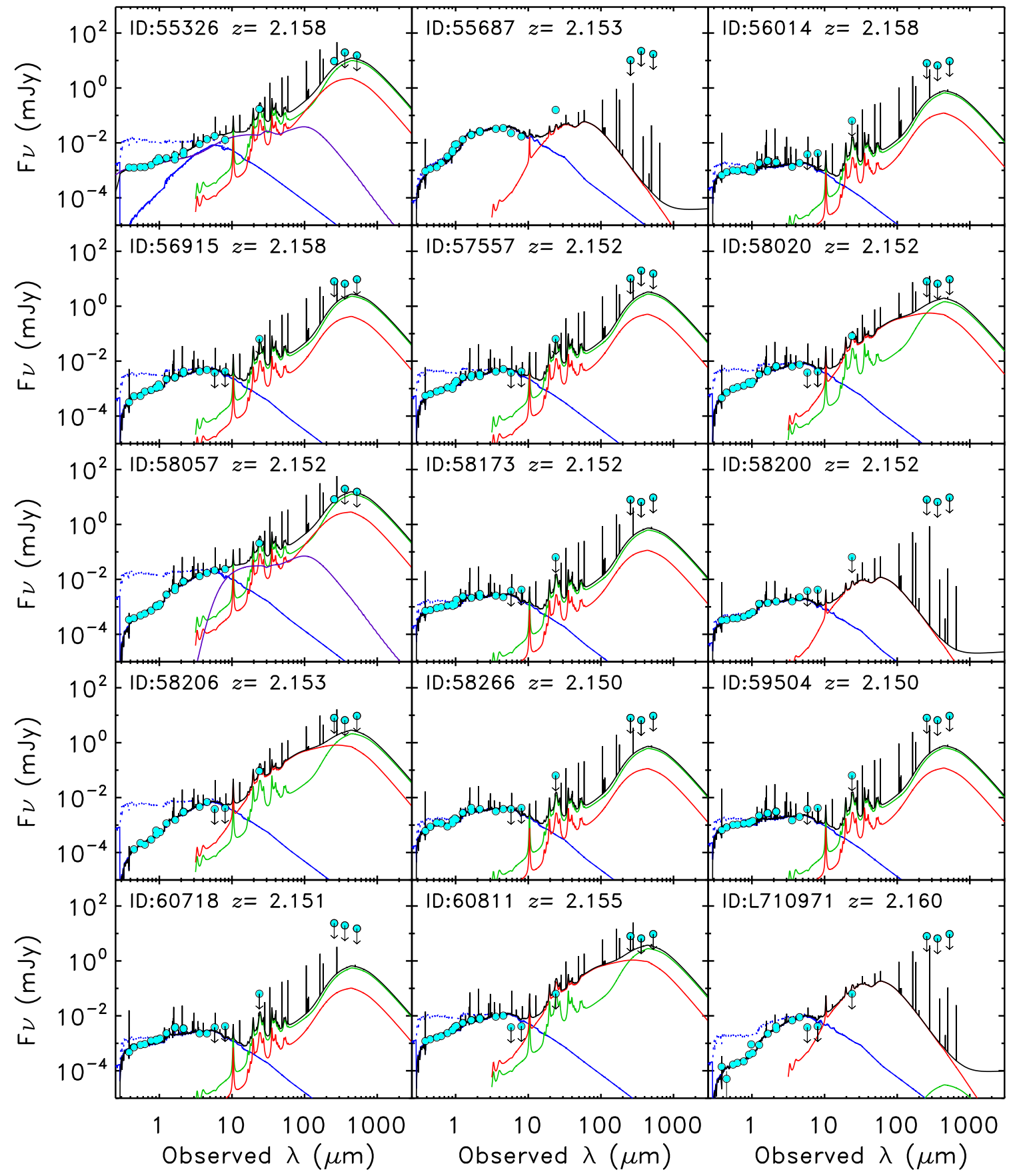

Fig. E.1. Observed spectral energy distributions (full cyan circles) and best fit templates (black lines) obtained with CIGALE at the spectroscopic redshift. Downward arrows are $5 \sigma$ upper limits. The blue solid and dotted lines represent the stellar component after and before intrinsic extinction is applied. The green and red lines represent emission from dust. The purple line represents an AGN component, present only in IDs 55326, and 58057. Source ID and spectroscopic redshifts are annotated. 


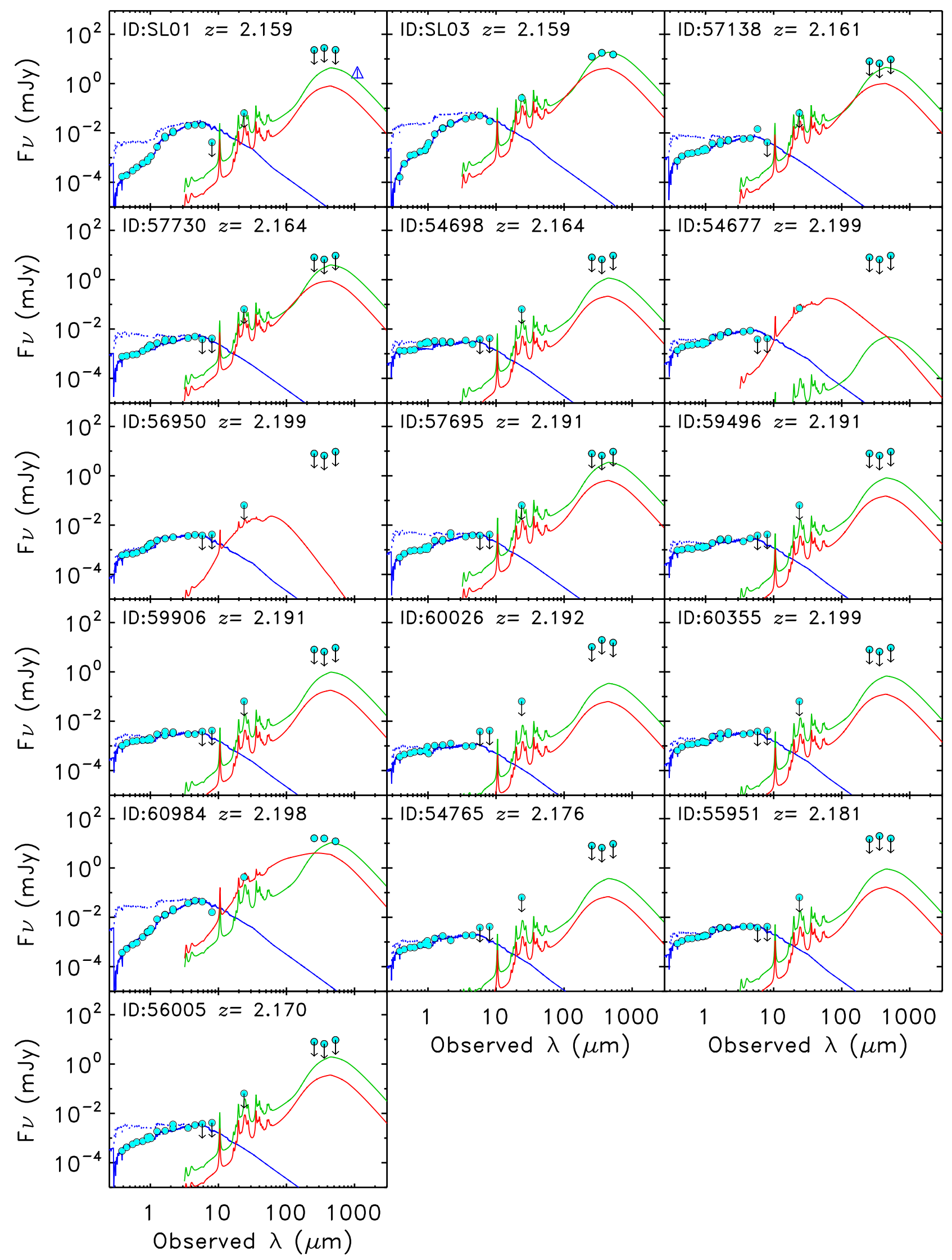

Fig. E.1. continued. 